\title{
Diffuse optical mammotomography: state-of-the-art and prospects
}

\author{
Alexander B. Konovalov ${ }^{1^{*}}$, Elina A. Genina ${ }^{2,3}$, and Alexey N. Bashkatov ${ }^{2,3}$ \\ ${ }^{1}$ Russian Federal Nuclear Center - Zababakhin Institute of Applied Physics, 13, Vasilieva Str., Snezhinsk, Chelyabinsk \\ Region, 456770, Russia \\ ${ }^{2}$ National Research Saratov State University, 83, Astrakhanskaya Str., Saratov, 410012, Russia \\ ${ }^{3}$ National Research Tomsk State University, 36, Lenina Pr., Tomsk, 634050, Russia \\ *e-mail: a konov@mail.vega-int.ru
}

Abstract: The principles of diffuse optical tomography (DOT) of tissues are presented. The DOT capabilities as a method of breast cancer diagnostics are analysed. The state-ofthe-art of the DOT instrumentation and methodological base in application to solving the mammography problems are described. The significant contribution of Russian scientists to the development of the DOT methodology is emphasised. Basing on the results of the analysis, the authors expect the possibility of soonest entry of diffuse optical mammotomographs to the market of medical imaging instrumentation, and the capability of Russian researchers to take part in the competition for this market. (C) 2016 Journal of Biomedical Photonics \& Engineering.

Key words: diffuse optical tomography, breast, optical and functional parameters, methods of determining optical parameters, methods of image reconstruction, perturbation model of reconstruction, temporal point spread function, spatial resolution, reconstruction time.

Paper \#2827 received 2015.12.20; revised manuscript received 2016.05.03; accepted for publication 2016.05.23; published online 2016.06.30. doi: 10.18287/JBPE16.02.020202

\section{References}

1. V. V. Tuchin, Tissue Optics: Light Scattering Methods and Instruments for Medical Diagnosis, 3rd ed., SPIE Press, Bellingham, WA (2015).

2. L. V. Wang, and H.-I. Wu, Biomedical Optics: Principles and Imaging, John Wiley \& Sons Inc., Hoboken, New Jersey (2007).

3. H. Jiang, Diffuse Optical Tomography: Principles and Applications, CRC Press, Boca Ration (2010).

4. V. V. Tuchin (ed.), Handbook of Photonics for Biomedical Science, CRC Press, Taylor \& Francis Group, London (2010).

5. A. Konovalov, Time-domain Diffuse Optical Mammotomography. The Photon Average Trajectory Method, Lambert Academic Publishing, Saarbrüken (2014) [in Russian]. ISBN: 978-3-659-53802-5.

6. I. J. Bigio, and S. Fantini, Quantitative Biomedical Optics. Theory, Methods, and Applications, Cambridge University Press, Cambridge (2016).

7. S. R. Arridge, and M. Schweiger, "Inverse methods for optical tomography," in Information Processing in Medical Imaging, H.H. Barrett (ed.), Springer-Verlag, Flagstaff, 259-277 (1993).

8. J. C. Hebden, S. R. Arridge, and D. T. Delpy, "Optical imaging in medicine: I. Experimental techniques," Phys. Med. Biol. 42, 825-840 (1997).

9. S. R. Arridge, and J. C. Hebden, “Optical imaging in medicine: II. Modelling and reconstruction,” Phys. Med. Biol. 42, 841-853 (1997).

10. B. B. Das, F. Liu, and R. R. Alfano, "Time-resolved fluorescence and photon migration studies in biomedical and random media,” Rep. Prog. Phys. 60, 227-292 (1997).

11. K. Michielsen, H. De'Raedt, J. Przeslawski, and N. Garcia, "Computer simulation of time-resolved optical imaging of objects hidden in turbid media," Phys. Rep. 304, 89-144 (1998).

12. S. R. Arridge, "Optical tomography in medical imaging," Inverse Problems 15, R41-R93 (1999). 
13. D. J. Hawrysz, and E. M. Sevick-Muraca, "Developments toward diagnostic breast cancer imaging using nearinfrared optical measurements and fluorescent contract agents," Neoplasia 2, 388-417 (2000).

14. D. A. Boas, D.H. Brooks, E. L. Miller, C. A. DiMarzio, M. Kilmer, and R. J. Gaudette, "Imaging the body with diffuse optical tomography," IEEE Sign. Proc. Mag. 18(6), 57-75 (2001).

15. D. A. Zimnyakov, and V. V. Tuchin, “Optical tomography of tissues," Quantum Electronics 32(10), 849-867 (2002).

16. A. G. Yodh, and D. A. Boas, "Functional imaging with diffusing light," Chap. 21 in Biomedical Photonics Handbook, T. Vo-Dinh (ed.), CRC Press, Boca Ration, 21-1-21-46 (2003).

17. A. Gibson, J. C. Hebden, and S. R. Arridge, "Recent advances in diffuse optical tomography," Phys. Med. Biol. 50, R1-R43 (2005).

18. D. R. Leff, O. J. Warren, L. C. Enfield, A. Gibson, T. Athanasiou, D. K. Patten, J. Hebden, G.Z. Yang, and A. Darzi, "Diffuse optical imaging of the healthy and diseased breast: a systematic review," Breast Cancer Research and Treatment 108, 9-22 (2008).

19. S. L. Jacques, and B. W. Pogue, "Tutorial on diffuse light transport,” J. Biomed. Opt. 13, 041302 (2008).

20. R. Choe, A. G. Yodh, "Diffuse optical tomography of the breast," Chap. 18 in Emerging Technology in Breast Imaging and Mammography, J. Suri, R. M. Rangayyan, and S. Laxminarayan (eds.), American Scientific Publishers, Stevenson Ranch, 317-342 (2008).

21. S. R. Arridge, and J. C. Schotland, “Optical tomography: forward and inverse problems,” Inverse Problems 25 , 123010 (2009).

22. T. Durduran, R. Choe, W. B. Baker, and A. G. Yodh, "Diffuse optics for tissue monitoring and tomography," Rep. Prog. Phys. 73, 076701 (2010).

23. M. Li, Y. Zhang, and J. Bai, "In vivo diffuse optical tomography and fluorescence molecular tomography," J. Healthcare Eng. 1, 477-507 (2010).

24. S. R. Arridge, "Methods in diffuse optical imaging," Phil. Trans. R. Soc. A. 369, 4558-4576 (2011).

25. T. D. O'Sullivan, A. E. Cerussi, D. J. Cuccia, and B. J. Tromberg, "Diffuse optical imaging using spatially and temporally modulated light,” J. Biomed. Opt. 17, 071311 (2012).

26. V. Venugopal, and X. Intes, "Recent advances in optical mammography," Current Medical Imaging Reviews 8(4), 244-259 (2012).

27. R. Choe, and T. Durduran, "Diffuse optical monitoring of the neoadjuvant breast cancer therapy," IEEE J. Sel. Top. Quantum Electron. 18(4), 1367-1386 (2012).

28. D. R. Busch, R. Choe, T. Durduran, and A. G. Yodh, "Towards non-invasive characterization of breast cancer and cancer metabolism with diffuse optics," PET Clin. 8(3), 345 (2013).

29. M. Schweiger, S. R. Arridge, and D. T. Delpy, "Application of the finite-element method for the forward and inverse models in optical tomography," J. Math. Imag. Vision 3, 263-283 (1993).

30. S. Nioka, M. Miwa, S. Orel, M. Shnall, M. Haida, S. Zhao, and B. Chance, "Optical imaging of human breast cancer," Adv. Exp. Med. Biol. 361, 171-179 (1994).

31. A. Yodh, and B. Chance, "Spectroscopy and imaging with diffusing light," Phys. Today 48, 34-40 (1995).

32. J. C. Hebden, S. R. Arridge, "Imaging through scattering media by the use of an analytical model of perturbation amplitudes in the time domain," Appl. Opt. 35, 6788-6796 (1996).

33. K. D. Paulsen, and H. Jiang, "Enhanced frequency-domain optical image reconstruction in tissues through total-variation minimization," Appl. Opt. 35, 3447-3458 (1996).

34. R. J. Grable, “Optical tomography improves mammography,” Laser Focus World 32, 113-118 (1996).

35. V. V. Lyubimov, "The physical foundation of the strongly scattering media laser tomography," Proc. SPIE 2769, 107-110 (1996).

36. V. V. Lyubimov, "Optical tomography of highly scattering media by using the first transmitted photons of ultrashort pulses," Optics and Spectroscopy 80(4), 616-619 (1996).

37. S. R. Arridge, and M. Schweiger, "Image reconstruction in optical tomography," Phil. Trans. R. Soc. Lond. B 352, 717-726 (1997).

38. S. B. Colak, D. G. Papaioannou, G.W. 't Hooft, M.B. van der Merk, H. Schomberg, J. C. Paasschens, J. B. Melissen, and N. A. van Asten, "Tomographic image reconstruction from optical projections in light-diffusing media," Appl. Opt. 36(1), 180-213 (1997).

39. Y. Pei, F.-B. Lin, and R. L. Barbour, "Modelling of sensitivity and resolution to an included object in homogeneous scattering media and in MRI-derived breast maps," Opt. Express 5, 203-219 (1999).

40. A. H. Hielscher, A. D. Klose, and K. M. Hanson, "Gradient-based iterative image reconstruction scheme for time-resolved optical tomography," IEEE Trans. Med. Imag. 18, 262-271 (1999).

41. A. D. Klose, and A. H. Hielscher, "Iterative reconstruction scheme for optical tomography based on the equation of radiative transfer," Med. Phys. 26, 1698-1707 (1999).

42. J. C. Ye, C. A. Bouman, K. J. Webb, and R. P. Millane, "Optical diffusion tomography using iterative coordinate descent optimization in a Bayesian framework,” J. Opt. Soc. Am. A 16, 2400-2412 (1999). 
43. V. B. Volkonskii, O. V. Kravtsenyuk, V. V. Lyubimov, E. P. Mironov, and A. G. Murzin, "The use of statistical characteristics of photon trajectories for the tomographic studies of optical macroheterogeneities in strongly scattering objects," Optics and Spectroscopy 86(2), 253-260 (1999).

44. D. A. Chursin, V. V. Shuvalov, and I. V. Shutov, "Optical tomograph with photon counting and projective reconstruction of the parameters of absorbing 'phantoms' in extended scattering media," Quantum Electronics 29(10), 921-926 (1999).

45. S. R. Arridge, J. C. Hebden, M. Schweiger, F. E. W. Schmidt, M. E. Fry, E. M. C. Hillman, H. Dehghani, and D. T. Delpy, "A method for three-dimensional time-resolved optical tomography," Int. J. Imaging Syst. Technol. 11, 2-11 (2000).

46. O. V. Kravtsenyuk, and V. V. Lyubimov, "Specific features of statistical characteristics of photon trajectories in a strongly scattering medium near an object surface," Optics and Spectroscopy 88(4), 608-614 (2000).

47. O. V. Kravtsenyuk, and V. V. Lyubimov, "Application of the method of smooth perturbations to the solution of problems of optical tomography of strongly scattering objects containing absorbing microinhomogeneities," Optics and Spectroscopy 89(1), 107-112 (2000).

48. J. C. Hebden, H. Veenstra, H. Dehghani, E. M. C. Hillman, M. Schweiger, S. R. Arridge, and D. T. Delpy, "Three-dimensional time-resolved optical tomography of a conical breast phantom," Appl. Opt. 40(19), 3278-3287 (2001).

49. V. Ntziachristos, A. H. Hielscher, A. G. Yodh, and B. Chance, "Diffuse optical tomography of highly heterogeneous media," IEEE Transactions on Medical Imaging 20(6), 470-478 (2001).

50. J. C. Ye, C. A. Bouman, K. J. Webb, and R. P. Millane, "Nonlinear multigrid algorithms for Bayesian optical diffusion tomography,” IEEE Trans. Image Proc. 10, 909-922 (2001).

51. R. L. Barbour, R. J. Grable, Y. Pei, S. Zhong, and C. H. Schmitz, "Optical tomographic imaging of dynamic features of dense-scattering media,” J. Opt. Soc. Am. A 18, 3018-3036 (2001).

52. V. A. Markel, and J. C. Schotland, "Inverse problem in optical diffusion tomography. I. Fourier-Laplace inversion formulas,” J. Opt. Soc. Am. A 18, 1336-1347 (2001).

53. V. V. Shuvalov, D. A. Chursin, and I. V. Shutov, "Spatial resolution, measuring time, and fast visualization of hidden deep phantoms in diffusion optical tomography of extended scattering objects," Laser Physics 11, 636649 (2001).

54. E. V. Tret'yakov, V. V. Shuvalov, and I. V. Shutov, "Fast approximate statistical nonlinear algorithms for diffusion optical tomography of objects with a complicated internal structure," Quantum Electronics 31(12), 1095-1100 (2001).

55. A. D. Klose, and A. H. Hielscher, "Optical tomography using the time-independent equation of radiative transfer: part II. Inverse model,” J. Quant. Spectrosc. Radiat. Transfer 72, 715-732 (2002).

56. V. A. Markel, and J. C. Schotland, "Inverse problem in optical diffusion tomography. II. Role of boundary conditions," J. Opt. Soc. Am. A 19, 558-566 (2002).

57. V. V. Lyubimov, A. G. Kalintsev, A. B. Konovalov, O. V. Lyamtsev, O. V. Kravtsenyuk, A. G. Murzin, O.V. Golubkina, G. B. Mordvinov, L. N. Soms, and L. M. Yavorskaya, "Application of the photon average trajectories method to real-time reconstruction of tissue inhomogeneities in diffuse optical tomography of strongly scattering media," Phys. Med. Biol. 47, 2109-2128 (2002).

58. E. V. Tret'yakov, V. V. Shuvalov, and I. V. Shutov, "Visualisation of details of a complicated inner structure of model objects by the method of diffusion optical tomography," Quantum Electronics 32(11), 941-944 (2002).

59. D. A. Boas, C. A. Bouman, and K. J. Webb (eds.), Special section on imaging through scattering media, J. Electron. Imaging 12(4), 581-620 (2003).

60. S. Jiang, B. W. Pogue, T. O. McBride, and K. D. Paulsen, "Quantitative analysis of near-infrared tomography: sensitivity to the tissue-simulating precalibration phantom," J. Biomed. Opt. 8, 308-315 (2003).

61. B. Brooksby, H. Dehghani, B. W. Pogue, and K. D. Paulsen, "Infrared (NIR) tomography breast image reconstruction with a priori structural information from MRI: algorithm development for reconstructing heterogeneities," IEEE J. Sel. Top. Quantum Electron. 9, 199-209 (2003).

62. A. D. Klose, and A. H. Hielscher, "Quasi-Newton methods in optical tomographic image reconstruction," Inverse Problems 19, 387-409 (2003).

63. M. Schweiger, A. P. Gibson, and S. R. Arridge, "Computational aspects of diffuse optical tomography," IEEE Comput. Sci. Eng. 5(6), 33-41 (2003).

64. V. A. Markel, V. Mital, and J. C. Schotland, "Inverse problem in optical diffusion tomography. III. Inversion formulas and singular-value decomposition," J. Opt. Soc. Am. A 20, 890-902 (2003).

65. V. A. Markel, J. A. O’Sullivan, and J. C. Schotland, "Inverse problem in optical diffusion tomography. IV. Nonlinear inversion formulas," J. Opt. Soc. Am. A 20, 903-912 (2003).

66. V. V. Lyubimov, O. V. Kravtsenyuk, A. G. Kalintsev, A. G. Murzin, L. N. Soms, A. B. Konovalov, I. I. Kutuzov, O. V. Golubkina, and L. M. Yavorskaya, "The possibility of increasing the spatial resolution in diffusion optical tomography," J. Opt. Technol. 70(10), 715-720 (2003). 
67. H. Dehghani, M. M. Doyley, B. W. Pogue, S. Jiang, J. Geng, and K. D. Paulsen, "Breast deformation modelling for image reconstruction in near infrared optical tomography," Phys. Med. Biol. 49, 1131-1145 (2004).

68. X. Intes, C. Maloux, M. Guven, B. Yazici, and B. Chance, "Diffuse optical tomography with physiological and spatial a priori constraints,” Phys. Med. Biol. 49, N155-N163 (2004).

69. V. A. Markel, and J. C. Schotland, "Symmetries, inversion formulas, and image reconstruction for optical tomography," Phys. Rev. E 70, 056616 (2004).

70. M. Schweiger, S. R. Arridge, and I. Nissilä, "Gauss-Newton method for image reconstruction in diffuse optical tomography,” Phys. Med. Biol. 50, 2365-2386 (2005).

71. T. Tarvainen, V. Kolehmainen, M. Vauhkonen, A. Vanne, A. P. Gibson, M. Schweiger, S. R. Arridge, and J. P. Kaipio, “Computational calibration method for optical tomography," Appl. Opt. 44(10), 1879-1888 (2005).

72. V. A. Markel, and J. C. Schotland, "Multiple projection optical diffusion tomography with plane wave illumination,” Phys. Med. Biol. 50, 2351-2364 (2005).

73. M. Guven, B. Yazici, X. Intes, and B. Chance, "Diffuse optical tomography with a priory anatomical information," Phys. Med. Biol. 50, 2837-2858 (2005).

74. J. Bai, T. Gao, K. Ying, and N. Chen, "Locating inhomogeneities in tissue by using the most probable diffuse path of light," J. Biomed. Opt. 10, 024024 (2005).

75. A. G. Kalintsev, N. A. Kalintseva, O. V. Kravtsenyuk, and V. V. Lyubimov, "Superresolution in diffuse optical tomography," Optics and Spectroscopy 99(1), 152-157 (2005).

76. S. R. Arridge, J. P. Kaipio, V. Kolehmainen, M. Schweiger, E. Somersalo, T. Tarvainen, and M. Vauhkonen, "Approximation errors and model reduction with an application in optical diffusion tomography," Inverse Problems 22(1), 175-195 (2006).

77. B. W. Pogue, S. C. Davis, X. Song, B. A. Brooksby, H. Dehghani, and K. D. Paulsen, "Image analysis methods for diffuse optical tomography," J. Biomed. Opt. 11(3), 033001 (2006).

78. B. W. Pogue, and M. S. Patterson, "Review of tissue simulating phantoms for optical spectroscopy, imaging and dosimetry," J. Biomed. Opt. 11, 044102 (2006).

79. O. V. Kravtsenyuk, V. L. Kuz'min, V. V. Lyubimov, and I. V. Meglinskii, "Diffuse optical tomography of dynamic inhomogeneities in randomly inhomogeneous media," Optics and Spectroscopy 100(6), 950-957 (2006).

80. A. B. Konovalov, V. V. Vlasov, A. G. Kalintsev, O. V. Kravtsenyuk, and V. V. Lyubimov, "Time-domain diffuse optical tomography using analytic statistical characteristics of photon trajectories," Quantum Electronics 36(11), 1048-1055 (2006).

81. G. Boverman, Q. Fang, S. A. Carp, E. L. Miller, D. H. Brooks, J. Selb, R. H. Moore, D. B. Kopans, and D. A. Boas, "Spatio-temporal imaging of the hemoglobin in the compressed breast with diffuse optical tomography," Phys. Med. Biol. 52, 3619-3641 (2007).

82. J. C. Schotland, and V. A. Markel, "Fourier-Laplace structure of the inverse scattering problem for the radiative transport equation," Inverse Problems and Imaging 1, 181-188 (2007).

83. A. B. Konovalov, D. V. Mogilenskikh, V. V. Vlasov, and A. N. Kiselev, "Algebraic reconstruction and postprocessing in incomplete data computed tomography: from X-rays to laser beams," Chap. 26 in Vision Systems: Applications, G. Obinata, and A. Dutta (eds.), I-Tech Education and Publishing, Vienna, 487-518 (2007).

84. A. B. Konovalov, V. V. Vlasov, D. V. Mogilenskikh, O. V. Kravtsenyuk, and V. V. Lyubimov, "Algebraic reconstruction and postprocessing in one-step diffuse optical tomography," Quantum Electronics 38(6), 588-596 (2008).

85. B. J. Tromberg, B. W. Pogue, K. D. Paulsen, A. G. Yodh, D. A. Boas, and A. E. Cerussi, “Assessing the future of diffuse optical imaging technologies for breast cancer management," Med. Phys. 35(6), 2443-2451 (2008).

86. R. Ziegler, B. Brendel, A. Schipper, R. Harbers, M. Beek, H. Rinneberg, and T. Nielsen, "Investigation of detection limits for diffuse optical tomography systems: I. Theory and experiment," Phys. Med. Biol. 54(2), 399-412 (2009).

87. R. Ziegler, B. Brendel, H. Rinneberg, and T. Nielsen, "Investigation of detection limits for diffuse optical tomography systems: II. Analysis of slab and cup geometry for breast imaging," Phys. Med. Biol. 54, 413-431 (2009).

88. J. Prakash, V. Chandrasekharan, V. Upendra, and P. K. Yalavarthy, "Accelerating frequency-domain diffuse optical tomographic image reconstruction using graphics processing units," J. Biomed. Opt. 15, 066009 (2010).

89. M. Patachia, D. C. A. Dutu, and D. C. Dumitras, "Blood oxygenation monitoring by diffuse optical tomography," Quantum Electronics 40(12), 1062-1066 (2010).

90. F. Larusson, S. Fantini, and E. L. Miller, "Hyperspectral image reconstruction for diffuse optical tomography," Biomed. Opt. Express 2(4), 946-965 (2011). 
91. M. Schweiger, "GPU-accelerated finite element method for modelling light transport in diffuse optical tomography,” Int. J. Biomed. Imaging 2011, 403892 (2011).

92. S. G. Proskurin, "Using late arriving photons for diffuse optical tomography of biological objects," Quantum Electronics 41(5), 402-406 (2011).

93. A. B. Konovalov, V. V. Vlasov, A. S. Uglov, and V. V. Lyubimov, “A semi-analytical perturbation model for diffusion tomogram reconstruction from time-resolved optical projections," Proc. SPIE 8088, 80880T (2011).

94. L. Y. Chen, M. C. Pan, and M. C. Pan, "Implementation of edge-preserving regularization for frequencydomain diffuse optical tomography," Appl. Opt. 51(1), 43-54 (2012).

95. O. Balima, Y. Favennec, J. Boulanger, and A. Charette, "Optical tomography with discontinuous Galerkin formulation of the radiative transfer equation in frequency domain,” J. Quant. Spectrosc. Radiat. Transfer 113, 805-814 (2012).

96. F. Larusson, S. Fantini, and E. L. Miller, "Parametric level set reconstruction methods for hyperspectral diffuse optical tomography,” Biomed. Opt. Express 3(5), 1006-1024 (2012).

97. F. Larusson, P. G. Anderson, E. Rosenberg, M. E. Kilmer, A. Sassaroli, S. Fantini, and E. L. Miller, "Parametric estimation of 3D tubular structures for diffuse optical tomography," Biomed. Opt. Express 4(2), 271-286 (2013).

98. A. B. Konovalov, V. V. Vlasov, and V. V. Lyubimov, "Statistical characteristics of photon distributions in a semi-infinite turbid medium: analytical expressions and their application to optical tomography," Optik 124, 6000-6008 (2013).

99. S. G. Proskurin, and A. Y. Potlov, "Early- and late-arriving photons in diffuse optical tomography," Photon. Las. Med. 2, 139-145 (2013).

100.B. Tavakoli, and Q. Zhu, "Two-step reconstruction method using global optimization and conjugate gradient for ultrasound-guided diffuse optical tomography," J. Biomed. Opt. 18(1), 016006 (2013).

101.J.M. Kainerstorfer, Y. Yu, G. Weliwitigoda, P.G. Anderson, A. Sassaroli, and S. Fantini, "Depth discrimination in diffuse optical transmission imaging by planar scanning off-axis fibers: initial applications to optical mammography," Plos One 8(3), e58510 (2013).

102.A. Y. Potlov, S. G. Proskurin, and S. V. Frolov, "Three-dimensional representation of late-arriving photons for detecting inhomogeneities in diffuse optical tomography," Quantum Electronics 44(2), 174-181 (2014).

103.M. J. Saikia, R. Kanhirodan, and R. M. Vasu, "High-speed GPU-based fully three-dimensional diffuse optical tomographic system,” Int. J. Biomed. Imaging 2014, 376456 (2014).

104.A. B. Konovalov, and V. V. Vlasov, "Theoretical limit of spatial resolution in diffuse optical tomography using a perturbation model," Quantum Electronics 44(3), 239-246 (2014).

105.A. S. Kuratov, K. V. Rudenko, and V. V. Shuvalov, "Differential visualisation of a spectrally selective structure of strongly scattering objects," Quantum Electronics 44(7), 652-656 (2014).

106.A. B. Konovalov, and V. V. Vlasov, "Calculation of the weighting functions for the reconstruction of absorbing inhomogeneities in tissue by time-resolved optical projections," Quantum Electronics 44(8), 719725 (2014).

107.T. Zhang, J. Zhou, P. R. Carney, and H. Jiang, "Towards real-time detection of seizures in awake rats with GPU accelerated diffuse optical tomography," J. Neurosci. Methods 240, 28-36 (2015).

108.L. Y. Chen, M. C. Pan, J. M. Yu, and M. C. Pan, "Diffuse optical imaging through incorporating structural information into edge-preserving regularization," Optical and Quantum Electronics 48(2), 130 (2016).

109.A. Y. Potlov, S. V. Frolov, and S. G. Proskurin, "Localization of inhomogeneities in diffuse optical tomography based on late arriving photons," Optics and Spectroscopy 120(1), 9-19 (2016).

110.A. B. Konovalov, and V. V. Vlasov, "Total variation based reconstruction of scattering inhomogeneities in tissue from time-resolved optical projections," Proc. SPIE 9917, 99170S (2016).

111.M. A. Franceschini, K. T. Moesta, S. Fantini, G. Gaida, E. Gratton, H. Jess, W. W. Mantulin, M. Seeber, P. M. Schlag, and M. Kaschke, "Frequency-domain techniques enhance optical mammography: initial clinical results," Proc. Natl. Acad. Sci. USA 94, 6468-6473 (1997).

112.J. H. Hoogenraad, M. B. van der Mark, S. B. Colak, G. W. t’Hooft, and E. S. van der Linden, "First results from the Philips optical mammoscope," Proc. SPIE 3194, 184-190 (1999).

113.S. Fantini, S. A. Walker, M. A. Franceschini, M. Kaschke, P. M. Schlag, and K. T. Moesta, “Assessment of the size, position, and optical properties of breast tumors in vivo by noninvasive optical methods," Appl. Opt. 37(10), 1982-1989 (1998).

114.S. B. Colak, M. B. van der Mark, G. W. 't Hooft, J. H. Hoogenraad, E. S. van der Linden, and F. A. Kuijpers, "Clinical optical tomography and NIR spectroscopy for breast cancer detection," IEEE J. Sel. Top. Quantum. Electron. 5(4), 1143-1158 (1999).

115.D. Grosenick, H. Wabnitz, H. H. Rinneberg, K. T. Moesta, and P. M. Schlag, "Development of a time-domain optical mammograph and first in vivo applications," Appl. Opt. 38(13), 2927-2943 (1999). 
116.T. O. McBride, B. W. Pogue, E. D. Gerety, S. B. Poplack, U. L. Osterberg, and K. D. Paulsen, “Spectroscopic diffuse optical tomography for the quantitative assessment of hemoglobin concentration and oxygen saturation in breast tissue," Appl. Opt. 38(25), 5480-5490 (1999).

117. V. Ntziachristos, A. G. Yodh, M. Schnall, and B. Chance, "Concurrent MRI and diffuse optical tomography of breast after indocyanine green enhancement,” Proc. Natl. Acad. Sci. USA 97, 2767-2772 (2000).

118.B. W. Pogue, S. P. Poplack, T. O. McBride, W. A. Wells, K. S. Osterman, U. L. Osterberg, and K. D. Paulsen, "Quantitative hemoglobin tomography with diffuse near-infrared spectroscopy: pilot results in the breast," Radiology 218, 261-266 (2001).

119.H. Jiang, Y. Xu, N. Iftimia, J. Eggert, K. Klove, L. Baron, and L. Fajardo, "Three-dimensional optical tomographic imaging of breast in a human subject," IEEE Trans. Med. Imag. 20(12), 1334-1340 (2001).

120.T. O. McBride, B. W. Pogue, S. Jiang, U. L. Osterberg, K. D. Paulsen, and S. P. Poplack, "Initial studies of in vivo absorbing and scattering heterogeneity in near-infrared tomographic breast imaging," Opt. Lett. 26(11), $822-824$ (2001).

121.V. Chernomordik, D. W. Hattery, D. Grosenick, H. Wabnitz, H. Rinneberg, K. T. Moesta, P. M. Schlag, and A. Gandjbakhche, "Quantification of optical properties of a breast tumor using random walk theory," J. Biomed. Opt. 7(1), 80-87 (2002).

122.T. O. McBride, B. W. Pogue, S. Poplack, S. Soho, W. A. Wells, S. Jiang, U. L. Osterberg, and K. D. Paulsen, "Multispectral near-infrared tomography: a case study in compensating for water and lipid content in hemoglobin imaging of the breast," J. Biomed. Opt. 7(1), 72-79 (2002).

123.J. P. Culver, R. Choe, M. J. Holboke, L. Zubkov, T. Durduran, A. Slemp, V. Ntziachristos, B. Chance, and A. G. Yodh, "Three-dimensional diffuse optical tomography in the parallel plane transmission geometry: evaluation of a hybrid frequency domain continuous wave clinical system for breast imaging," Med. Phys. 30(2), 235-247 (2003).

124.A. Li, E. L. Miller, M. E. Kilmer, T. J. Brukilacchio, T. Chaves, J. Scott, Q. Zhang, T. Wu, M. Chorlton, R. H. Moore, D. B. Kopans, and D. A. Boas, "Tomographic optical breast imaging guided by three-dimensional mammography," Appl. Opt. 42(25), 5181-5190 (2003).

125.H. Dehghani, B. W. Pogue, S. P. Poplack, and K. D. Paulsen, "Multiwavelength three-dimensional nearinfrared tomography of the breast: initial simulation, phantom, and clinical results," Appl. Opt. 42, 135-145 (2003).

126.S. Srinivasan, B. W. Pogue, S. Jiang, H. Dehghani, C. Kogel, S. Soho, J. J. Gibson, T. D. Tosteson, S. P. Poplack, and K. D. Paulsen, "Interpreting hemoglobin and water concentration, oxygen saturation, and scattering measured in vivo by near-infrared breast tomography," Proc. Natl. Acad. Sci. USA 100(21), 1234912354 (2003).

127.X. Intes, J. Ripoll, Y. Chen, S. Nioka, A. G. Yodh, and B. Chance, "In vivo continuous-wave optical breast imaging enhanced with indocyanine green," Med. Phys. 30(6), 1039-1047 (2003).

128. Y. Xu, X. Gu, L. L. Fajardo, and H. Jiang, "In vivo breast imaging with diffuse optical tomography based on higher-order diffusion equations," Appl. Opt. 42, 3163-3169 (2003).

129.D. Grosenick, K. T. Moesta, H. Wabnitz, J. Mucke, C. Stroszczynski, R. Macdonald, P. M. Schlag, and H. Rinneberg, "Time-domain optical mammography: initial clinical results on detection and characterization of breast tumors," Appl. Opt. 42(16), 3170-3186 (2003).

130.D. Grosenick, H. Wabnitz, K. T. Moesta, J. Mucke, M. Moller, C. Stroszczynski, J. Stobel, B. Wassermann, P. M. Schlag, and H. Rinneberg, "Concentration and oxygen saturation of haemoglobin of 50 breast tumours determined by time-domain optical mammography,” Phys. Med. Biol. 49, 1165-1181 (2004).

131.P. Taroni, A. Pifferi, A. Torricelli, L. Spinelli, G. M. Danesini, and R. Cubeddu, "Do shorter wavelengths improve contrast in optical mammography?," Phys. Med. Biol. 49, 1203-1215 (2004).

132.P. Taroni, G. Danesini, A. Torricelli, A. Pifferi, L. Spinelli, and R. Cubeddu, "Clinical trial of time-resolved scanning optical mammography at 4 wavelengths between 683 and 975 nm," J. Biomed. Opt. 9(3), 464-473 (2004).

133.S. Srinivasan, B. W. Pogue, H. Dehghani, S. Jiang, X. Song, and K. D. Paulsen, "Improved quantification of small objects in near-infrared diffuse optical tomography," J. Biomed. Opt. 9(6), 1161-1171 (2004).

134.B. W. Pogue, S. Jiang, H. Dehghani, C. Kogel, S. Soho, S. Srinivasan, X. Song, T. D. Tosteson, S. P. Poplack, and K. D. Paulsen, "Characterization of hemoglobin, water and NIR scattering in breast tissue: analysis of intersubject variability and menstrual cycle changes," J. Biomed. Opt. 9(3), 541-552 (2004).

135.J. C. Hebden, and H. Rinneberg (eds.), Special section on time-domain optical mammography, Phys. Med. Biol. 50(11), 2429-2596 (2005).

136.Q. Zhang, T. J. Brukilacchio, A. Li, J. J. Stott, T. Chaves, E. Hillman, T. Wu, M. Chorlton, E. Rafferty, R. H. Moore, D. B. Kopans, and D. A. Boas, "Coregistered tomographic X-ray and optical breast imaging: initial results," J. Biomed. Opt. 10(2), 024033 (2005).

137.T. D. Yates, J. C. Hebden, A. P. Gibson, L. Enfield, N. L. Everdell, S. R. Arridge, and D. T. Delpy, “Timeresolved optical mammography using a liquid coupled interface,” J. Biomed. Opt. 10(5), 054011 (2005). 
138.R. Choe, A. Corlu, K. Lee, T. Durduran, S. D. Konecky, M. Grosicka-Koptyra, S. R. Arridge, B. J. Czerniecki, D. L. Fraker, A. DeMichele, B. Chance, M. A. Rosen, and A. G. Yodh, "Diffuse optical tomography of breast cancer during neoadjuvant chemotherapy: A case study with comparison to MRI," Med. Phys. 32(4), 11281139 (2005).

139.B. Brooksby, B. W. Pogue, S. Jiang, H. Dehghani, S. Srinivasan, C. Kogel, T. D. Tosteson, J. Weaver, S. P. Poplack, and K. D. Paulsen, "Imaging breast adipose and fibroglandular tissue molecular signatures by using hybrid MRI-guided near-infrared spectral tomography," Proc. Natl. Acad. Sci. USA 103(23), 8828-8833 (2006).

140.L. C. Enfield, A. P. Gibson, N. L. Everdell, D. T. Delpy, M. Schweiger, S. R. Arridge, C. Richardson, M. Keshtgar, M. Douek, and J. C. Hebden, "Three-dimensional time-resolved optical mammography of the uncompressed breast," Appl. Opt. 46(17), 3628-3638 (2007).

141.S. Srinivasan, B. W. Pogue, C. Carpenter, P. K. Yalavarthy, and K. Paulsen, “A boundary element approach for image-guided near-infrared absorption and scatter estimation," Med. Phys. 34(11), 4545-4557 (2007).

142.J. Z. Wang, X. Liang, Q. Zhang, L. L. Fajardo, and H. Jiang, “Automated breast cancer classification using near-infrared optical tomographic images,” J. Biomed. Opt. 13(4), 044001 (2008).

143.A. G. Orlova, I. V. Turchin, V. I. Plehanov, N.M. Shakhova, I. I. Fiks, M. I. Kleshnin, N. Y. Konuchenko, and V. A. Kamensky, "Frequency-domain diffuse optical tomography with single source-detector pair for breast cancer detection," Laser Phys. Lett. 5(4), 321-327 (2008).

144.R. Choe, S. D. Konecky, A. Corlu, K. Lee, T. Durduran, D. R. Busch, S. Pathak, B. J. Czerniecki, J. Tchou, D. L. Fraker, A. Demichele, B. Chance, S. R. Arridge, M. Schweiger, J. P. Culver, M. D. Schnall, M. E. Putt, M. A. Rosen, and A. G. Yodh, "Differentiation of benign and malignant breast tumors by in-vivo threedimensional parallel-plate diffuse optical tomography," J. Biomed. Opt. 14(2), 024020 (2009).

145.J. Wang, B. W. Pogue, S. Jiang, and K. D. Paulsen, "Near-infrared tomography of breast cancer hemoglobin, water, lipid, and scattering using combined frequency domain and CW measurements," Opt. Lett. 35, 82-84 (2010).

146.Q. Zhu, P. U. Hegde, A. Ricci, M. Kane, E. B. Cronin, Y. Ardeshirpour, C. Xu, A. Aguirre, S. H. Kurtzman, P. J. Deckers, and S. H. Tannenbaum, "The potential role of optical tomography with ultrasound localization in assisting ultrasound diagnosis of early-stage invasive breast cancers," Radiology 256(2), 367-378 (2010).

147.Y. Yu, A. Sassaroli, D. K. Chen, M. J. Homer, R. A. Graham, and S. Fantini, "Near-infrared, broad-band spectral imaging of the human breast for quantitative oximetry: applications to healthy and cancerous breasts," J. Innov. Opt. Health Sci. 3(4), 267-277 (2010).

148.D. R. Busch, W. Guo, R. Choe, T. Durduran, M. D. Feldman, C. Mies, M. A. Rosen, M. D. Schnall, B. J. Czerniecki, J. Tchou, A. DeMichele, M. E. Putt, and A. G. Yodh, "Computer aided automatic detection of malignant lesions in diffuse optical mammography,” Med. Phys. 37(4), 1840-1849 (2010).

149.S. Srinivasan, C. M. Carpenter, H. R. Ghadyani, S. J. Taka, P. A. Kaufman, R. M. DiFlorio-Alexander, W. A. Wells, B. W. Pogue, and K. D. Paulsen, "Image guided near-infrared spectroscopy of breast tissue in vivo using boundary element method,” J. Biomed. Opt. 15(6), 061703 (2010).

150.Q. Q. Fang, J. Selb, S. A. Carp, G. Boverman, E. L. Miller, D. H. Brooks, R. H. Moore, D. B. Kopans, and D. A. Boas, "Combined optical and X-ray tomosynthesis breast imaging," Radiology 258, 89-97 (2011).

151. Y. Ueda, K. Yoshimoto, E. Ohmae, T. Suzuki, T. Yamanaka, D. Yamashita, H. Ogura, C. Teruya, H. Nasu, E. Imi, H. Sakahara, M. Oda, and Y. Yamashita, "Time-resolved optical mammography and its preliminary clinical results,” Technol. Cancer Res. Treat. 10(5), 393-401 (2011).

152.S. Fantini, and A. Sassaroli, "Near-infrared optical mammography for breast cancer detection with intrinsic contrast," Ann. Biomed. Eng. 40(2), 398-407 (2012).

153.Q. Zhu, P. A. DeFusco, A. Ricci, E. B. Cronin, P. U. Hegde, M. Kane, B. Tavakoli, Y. Xu, J. Hart, and S. H. Tannenbaum, "Breast cancer: assessing response to neoadjuvant chemotherapy by using US-guided nearinfrared tomography," Radiology 266(2), 433-442 (2013).

154.L. Enfield, G. Cantanhede, M. Douek, V. Ramalingam, A. Purushotham, J. Hebden, and A. Gibson, "Monitoring the response to neoadjuvant hormone therapy for locally advanced breast cancer using threedimensional time-resolved optical mammography," J. Biomed. Opt. 18(5), 056012 (2013).

155.P. G. Anderson, J. M. Kainerstorfer, A. Sassaroli, N. Krishnamurthy, M. J. Homer, R.A. Graham, and S. Fantini, "Broadband optical mammography: chromophore concentration and hemoglobin saturation contrast in breast cancer," Plos One 10(3), e0117322 (2015).

156.H. Ogura, N. Yoshizawa, S. Ueda, Y. Hosokawa, R. Matsunuma, J. Tochikubo, H. Nasu, T. Shigekawa, H. Takeuchi, A. Osaki, T. Saeki, K. Yoshimoto, E. Ohmae, T. Suzuki, Y. Ueda, Y. Yamashita, and H. Sakahara, "Near-infrared diffuse optical imaging for early prediction to neoadjuvant chemotherapy in patients with primary breast cancer," Cancer Res. 76, P4-03-06 (2016).

157.M. J. Kim, M.-Y. Su, H. J. Yu, J.-H. Chen, E.-K. Kim, H. J. Moon, and J. S. Choi, "US-localized diffuse optical tomography in breast cancer: comparison with pharmacokinetic parameters of DCE-MRI and with pathologic biomarkers," BMC Cancer 16, 50 (2016). 
158.R. Gordon, "Stop breast cancer now! Imagining imaging pathways toward search, destroy, cure, and watchful waiting of premetastasis breast cancer," Chap. 10 in Breast Cancer: A Lobar Disease, T. Tot. (ed.), SpringerVerlag, London, 167-205 (2011).

159.I. V. Turchin, V. A. Kamensky, V. I. Plehanov, A. G. Orlova, M. S. Kleshnin, I. I. Fiks, M. V. Shirmanova, I. G. Meerovich, L. R. Arslanbaeva, V. V. Jerdeva, and A. P. Savitsky, "Fluorescence diffuse tomography for detection of red fluorescent protein expressed tumors in small animals," J. Biomed. Opt. 13(4), 041310 (2008).

160.M. S. Kleshnin, and I. V. Turchin, "Spectrally resolved fluorescence diffuse tomography of biological tissues," Quantum Electronics 40(6), 531-537 (2010).

161.M. S. Kleshnin, and I. V. Turchin, "Fluorescence diffuse tomography technique with autofluorescence removal based on dispersion of biotissue optical properties," Laser Phys. Lett. 10, 075601 (2013).

162.V. V. Tuchin, S. S. Utz, and I. V. Yaroslavsky, “Tissue optics, light distribution, and spectroscopy,” Opt. Eng. 33(10), 3178-3188 (1994).

163. V. V. Tuchin, "Light-tissue interactions," Chap. 3 in Biomedical Photonics Handbook, T. Vo-Dinh (ed.), CRC Press, Boca Ration (2003).

164.A. N. Bashkatov, E. A. Genina, and V. V. Tuchin, "Tissue Optical Properties," Chap. 5 in Handbook of Biomedical Optics, D. A. Boas, C. Pitris, and N. Ramanujam (eds.), CRC Press, Taylor \& Francis Group, London, 67-100 (2011).

165.A. N. Bashkatov, E. A. Genina, V. I. Kochubey, V. V. Tuchin, E. E. Chikina, A. B. Knyazev, and O. V. Mareev, "Optical properties of mucous membrane in the spectral range 350-2000 nm," Optics and Spectroscopy 97(6), 978-983 (2004).

166.A. N. Bashkatov, E. A. Genina, V. I. Kochubey, and V. V. Tuchin, "Optical properties of human skin, subcutaneous and mucous tissues in the wavelength range from 400 to $2000 \mathrm{~nm}$," Journal of Physics D: Applied Physics 38(15), 2543-2555 (2005).

167.A. N. Bashkatov, E. A. Genina, V. I. Kochubey, and V. V. Tuchin, "Optical properties of the subcutaneous adipose tissue in the spectral range 400-2500 nm," Optics and Spectroscopy 99(5), 836-842 (2005).

168.A. N. Bashkatov, E. A. Genina, V. I. Kochubey, A. A. Gavrilova, S. V. Kapralov, V. A. Grishaev, and V. V. Tuchin, "Optical properties of human stomach mucosa in the spectral range from 400 to 2000 nm: prognosis for gastroenterology," Medical Laser Application 22, 95-104 (2007).

169.A. N. Bashkatov, E. A. Genina, V. I. Kochubey, and V. V. Tuchin, "Optical properties of human sclera in spectral range 370-2500 nm," Optics and Spectroscopy 109(2), 197-204 (2010).

170.A. N. Bashkatov, E. A. Genina, and V. V. Tuchin, "Optical properties of skin, subcutaneous, and muscle tissues: a review," Journal of Innovative Optical Health Sciences 4(1), 9-38 (2011).

171.A. N. Bashkatov, E. A. Genina, V. I. Kochubey, V. S. Rubtsov, E. A. Kolesnikova, and V. V. Tuchin, “Optical properties of human colon tissues in the 350-2500 nm spectral range," Quantum Electronics 44(8), 779-784 (2014).

172. The Free Dictionary by Farlex.

173. Y. L. Zolotko, Atlas of Topographic Anatomy of Human. Part 2. Breast, Abdomen, Pelvis, Meditsina, Moscow (1967) [in Russian].

174. Topographic anatomy and operative surgery. Breast topography [in Russian].

175.V. P. Kharchenko, and N. I. Rozhkova (eds.) Mammology. National Handbook, GEOTAR-Media, Moscow (2009) [in Russian].

176.J. N. Wolfe, “Xerography of the breast,” Radiology 91, 231-240 (1968).

177.D. B. Kopans, Breast Imaging, 3rd ed., Lippincott Williams \& Wilkins, Philadelphia (2007).

178.P. Taroni, A. Pifferi, G. Quarto, L. Spinelli, A. Torricelli, F. Abbate, A. Villa, N. Balestreri, S. Menna, E. Cassano, and R. Cubeddu, "Noninvasive assessment of breast cancer risk using time-resolved diffuse optical spectroscopy," J. Biomed. Opt. 15(6), 060501 (2010).

179.A. Cerussi, N. Shah, D. Hsiang, A. Durkin, J. Butler, and B. J. Tromberg, "In vivo absorption, scattering, and physiologic properties of 58 malignant breast tumors determined by broadband diffuse optical spectroscopy," J. Biomed. Opt. 11(4), 044005 (2006).

180.T. L. Troy, D. L. Page, and E. M. Sevick-Muraca, "Optical properties of normal and diseased breast tissue: prognosis for optical mammography,” J. Biomed. Opt. 1(3), 342-355 (1996).

181.Histological Grading of Breast Cancer, $2^{\text {nd }}$ ed., Meditsina, Moscow (1984) [in Russian].

182.L. W. Dalton, D. L. Page, and W. D. Dupont, "Histologic grading of breast carcinoma: a reproducibility study," Cancer 73, 2765-2770 (1994).

183.M. Culter, "Transillumination as an aid in the diagnosis of breast lesions," Surg. Gynecol. Obstet. 48, 721-727 (1929).

184.C. M. Gros, Y. Quenneville, and Y. J. Hummel, “Diaphanologie mammaire,” J. Radiol. Electrol. Med. Nucl. 53, 297-306 (1972).

185.H. Wallberg, "Diaphanography in various breast disorders: clinical and experimental observations," Acta Radiol. Diagn. 26, 271-276 (1985). 
186.B. Drexler, J. L. Davis, and G. Schofield, “Diaphanography in the diagnosis of breast cancer,” Radiology 157 , 41-44 (1985).

187.A. Alveryd, I. Andersson, K. Aspegren, G. Balldin, N. Bjurstam, G. Edström, G. Fagerberg, U. Glas, O. Jarlman, S. A. Larsson, E. Lidbrink, H. Lingaas, M. Löfgren, C.-M. Rudenstam, L. Strender, L. Samuelsson, L. Tabàr, A. Taube, H. Wallberg, P. Åkesson, and D. Hallberg, "Light scanning versus mammography for detection of breast cancer in screening and clinical practice," Cancer 65(8), 1671-1677 (1990).

188.W.-F. Cheong, S. A. Prahl, and A. J. Welch, “A review of the optical properties of biological tissue,” IEEE J. Quantum Electronics 26(12), 2166-2185 (1990).

189.J. Mobley, and T. Vo-Dinh, "Optical properties of tissue," Chap. 2 in Biomedical Photonics Handbook, T. VoDinh (ed.), CRC Press, Boca Ration (2003).

190.V. G. Peters, D. R. Wyman, M. S. Patterson, and G. L. Frank, "Optical properties of normal and diseased human breast tissues in the visible and near infrared," Phys. Med. Biol. 35(9), 1317-1334 (1990).

191.S. P. Treweek, and J. C. Barbenel, "Direct measurement of the optical properties of human breast skin," Medical \& Biological Engineering \& Computing 34, 285-289 (1996).

192.Y. Zhang, Y. Chen, Y. Yu, X. Xue, V. V. Tuchin, and D. Zhu, "Visible and near-infrared spectroscopy for distinguishing malignant tumor tissue from benign tumor and normal breast tissues in vitro," J. Biomed. Opt. 18(7), 077003 (2013).

193.S. A. Prahl, M. J. C. van Gemert, and A. J. Welch, "Determining the optical properties of turbid media by using the adding-doubling method," Appl. Opt., 32(4), 559-568 (1993).

194.M. R. Neuman, "Pulse oximetry: physical principles, technical realization, and present limitations," Adv. Exp. Med. Biol. 220, 135-144 (1987).

195.J. W. Severinghaus, "History and recent developments in pulse oximetry," Scand. J. Clin. Lab. Invest. 53, 105-111 (1993).

196.G. Maret, and P. Wolf, "Multiple light scattering from disordered media. The effect of Brownian motion of scatterers," Zeitschrift fur Phisik B Condensed Matter 65, 409-413 (1987).

197.D. J. Pine, D. A. Weitz, P. M. Chaikin, and E. Herbolzheimer, "Diffusing-wave spectroscopy," Phys. Rev. Lett. 60, 1134-1137 (1988).

198.V. Ntziachristos, A. G. Yodh, M. Schnall, and B. Chance, "MRI-guided diffuse optical spectroscopy of malignant and benign breast lesions," Neoplasia 4(4), 347-354 (2002).

199.F. Bevilacqua, A. J. Berger, A. E. Cerussi, D. Jakubowski, and B. J. Tromberg, "Broadband absorption spectroscopy in turbid media by combined frequency-domain and steady-state methods," Appl. Opt. 39(34), 6498-6507 (2000).

200.S. H. Chung, A. E. Cerussi, C. Klifa, H. M. Baek, O. Birgul, G. Gulsen, S. I. Merritt, D. Hsiang, and B. J. Tromberg, "In vivo water state measurements in breast cancer using broadband diffuse optical spectroscopy," Phys. Med. Biol. 53, 6713-6727 (2008).

201.R. Cubeddu, C. D'Andrea, A. Pifferi, P. Taroni, A. Torricelli, and G. Valentini, "Effects of the menstrual cycle on the red and near-infrared optical properties of the human breast," Photochemistry and Photobiology 72(3), 383-391 (2000).

202.T. Durduran, R. Choe, J. P. Culver, L. Zubkov, M. J. Holboke, J. Giammarco, B. Chance, and A. G. Yodh, "Bulk optical properties of healthy female breast tissue," Phys. Med. Biol. 47, 2847-2861 (2002).

203.C. Zhu, G. M. Palmer, T. M. Breslin, J. Harter, and N. Ramanujam, "Diagnosis of breast cancer using fluorescence and diffuse reflectance spectroscopy: a Monte-Carlo-model-based approach," J. Biomed. Opt. 13(3), 034015 (2008).

204.R. L. P. van Veen, W. Verkruysse, and H. J. C. M. Sterenborg, "Diffuse-reflectance spectroscopy from 500 to $1060 \mathrm{~nm}$ by correction for inhomogeneously distributed absorbers," Opt. Lett. 27(4), 246-248 (2002).

205.B. J. Tromberg, N. Shah, R. Lanning, A. Cerussi, J. Espinoza, T. Pham, L. Svaasand, and J. Butler, "Noninvasive in vivo characterization of breast tumors using photon migration spectroscopy," Neoplasia 2(1-2), 2640 (2000).

206.P. Taroni, A. Pifferi, G. Quarto, L. Spinelli, A. Torricelli, F. Abbate, N. Balestreri, S. Ganino, S. Menna, E. Cassano, and R. Cubeddu, "Effects of tissue heterogeneity on the optical estimate of breast density," Biomed. Opt. Express 3(10), 2411-2418 (2012).

207.P. Taroni, A. Bassi, D. Comelli, A. Farina, R. Cubeddu, and A. Pifferi, "Diffuse optical spectroscopy of breast tissue extended to 1100 nm," J. Biomed. Opt. 14(5), 054030 (2009).

208.L. Spinelli, A. Torricelli, A. Pifferi, P. Taroni, G. M. Danesini, and R. Cubeddu, "Bulk optical properties and tissue components in the female breast from multiwavelength time-resolved optical mammography," J. Biomed. Opt. 9(6), 1137-1142 (2004).

209.N. Shah, A. E. Cerussi, D. Jakubowski, D. Hsiang, J. Butler, and B. J. Tromberg, "Spatial variations in optical and physiological properties of healthy breast tissue," J. Biomed. Opt. 9(3), 534-540 (2004).

210.J. L. Sandell, and T. C. Zhu, "A review of in-vivo optical properties oh human tissues and its impact on PDT," J. Biophotonics 4(11-12), 773-787 (2011). 
211.G. M. Palmer, and N. Ramanujam, "Monte Carlo-based inverse model for calculating tissue optical properties. Part II: Application to breast cancer diagnosis,” Appl. Opt. 45(5), 1072-1078 (2006).

212.D. Grosenick, H. Wabnitz, K. T. Moesta, J. Mucke, P. M. Schlag, and H. Rinneberg, "Time-domain scanning optical mammography: II. Optical properties and tissue parameters of 87 carcinomas," Phys. Med. Biol. 50, 2451-2468 (2005).

213.M. S. Nair, N. Ghosh, N. S. Raju, and A. Pradhan, "Determination of optical parameters of human breast tissue from spatially resolved fluorescence: a diffusion theory model," Appl. Opt. 41(19), 4024-4035 (2002).

214.W. Mo, T. S. S. Chan, L. Chen, and N. Chen, "Quantitative characterization of optical and physiological parameters in normal breasts using time-resolved spectroscopy: in vivo results of 19 Singapore women," J. Biomed. Opt. 14(6), 064004 (2009).

215.S. E. Skipetrov, and I. V. Meglinskii, "Diffusing-wave spectroscopy in randomly inhomogeneous media with spatially localized scattered flows," J. Exp. and Theor. Phys. 86(4), 661-665 (1998).

216.M. Heckmeier, S. E. Skipetrov, G. Maret, and R. Maynard, "Imaging of dynamic heterogeneities in multiplescattering media," J. Opt. Soc. Am. A 14, 185-191 (1997).

217.D. A. Boas, L. E. Campbell, and A. G. Yodh, "Scattering and imaging with diffusing temporal field correlations," Phys. Rev. Lett. 75, 1855-1858 (1995).

218.D. A. Boas, and A. G. Yodh, "Spatially varying dynamic properties of turbid media probed with diffusing temporal light correlation,” J. Opt. Soc. Am. A. 14, 192-215 (1997).

219.C. Zhou, G. Yu, D. Furuya, J. Greenberg, A. Yodh, and T. Durduran, "Diffuse optical correlation tomography of cerebral blood flow during cortical spreading depression in rat brain,” Opt. Express 14(3), 1125-1144 (2006).

220.H. M. Varma, B. Banerjee, D. Roy, A. K. Nandakumaran, and R.M. Vasu, "Convergence analysis of the Newton algorithm and a pseudo-time marching scheme for diffuse correlation tomography," J. Opt. Soc. Am. A 27(2), 259-267 (2010).

221. Y. Lin, C. Huang, D. Irwin, L. He, Y. Shang, and G. Yu, "Three-dimensional flow contrast imaging of deep tissue using noncontact diffuse correlation tomography," Appl. Phys. Lett. 104, 121103 (2014).

222.H. M. Varma, C. P. Valdes, A. K. Kristoffersen, J. P. Culver, and T. Durduran, "Speckle contrast optical tomography: A new method for deep tissue three-dimensional tomography of blood flow," Biomed. Opt. Express 5(4), 1275-1289 (2014).

223.L. He, Y. Lin, C. Huang, D. Irwin, M. M. Szabunio, and G. Yu, "Noncontact diffuse correlation tomography of human breast tumor," J. Biomed. Opt. 20(8), 086003 (2015).

224.E. M. C. Hillman, Experimental and theoretical investigations of near infrared tomographic imaging methods and clinical applications: Ph.D. thesis, University College London, London (2002).

225.B. J. Tromberg, A. Cerussi, N. Shah, M. Compton, A. Durkin, D. Hsiang, J. Butler, and R. Mehta, "Diffuse optics in breast cancer: detecting tumors in pre-menopausal women and monitoring neoadjuvant chemotherapy," Breast Cancer Res. 7(6), 279-285 (2005).

226.A. Torricelli, L. Spinelli, A. Pifferi, P. Taroni, R. Cubeddu, and G.M. Danesini, "Use of a nonlinear perturbation approach for in vivo breast lesion characterization by multiwavelength time-resolved optical mammography," Opt. Express 11, 853-867 (2003).

227.I. Fiks, M. Kleshnin, and I. Turchin, "Reconstruction in fluorescence diffuse tomography based on nonnegativity condition," Proc. SPIE 8799, 87990V (2013).

228.I. Fiks, "A novel method based on the Tikhonov functional for non-negative solution of a system of linear equations with non-negative coefficients,” Int. J. Comput. Methods 11, 1350071 (2014).

229.D. T. Delpy, M. Cope, P. van der Zee, S. Arridge, S. Wray, and J. Wyatt, "Estimation of optical pathlength through tissue from direct time of flight measurement," Phys. Med. Biol. 33(12), 1433-1442 (1988).

230.F. E. W. Schmidt, M. E. Fry, E. M. C. Hillman, J. C. Hebden, and D. T. Delpy, "A 32-channel time-resolved instrument for medical optical tomography," Rev. Sci. Instrum. 71(1), 256-265 (2000).

231.V. Ntziachristos, X. Ma, and B. Chance, "Time-correlated single photon counting imager for simultaneous magnetic resonance and near-infrared mammography," Rev. Sci. Instrum. 69, 4221-4233 (1998).

232.C. V. Zint, F. Gao, M. Torregrossa, and P. Poulet, "Near-infrared optical tomography of scattering cylindrical phantoms using time-resolved detection," Proc. SPIE 4250, 109-119 (2001).

233.S. Antonioli, M. Crotti, A. Cuccato, I. Rech, and M. Ghioni, “Time-correlated single-photon counting system based on a monolithic time-to-amplitude converter," J. Modern Opt. 59(17), 1512-1524 (2012).

234.S. Antonioli, L. Miari, A. Cuccato, M. Crotti, I. Rech, and M. Ghioni, "8-channel acquisition system for timecorrelated single-photon counting," Rev. Sci. Instrum., 84(6), 064705 (2013).

235. Y. Bérubé-Lauzière, M. Crotti, S. Boucher, S. Ettehadi, J. Pichette, and I. Rech, "Prospects on time-domain diffuse optical tomography based on time-correlated single photon counting for small animal imaging," J. Spectrosc. 2016, 1947613 (2016).

236.C. H. Schmitz, D. P. Klemer, R. Hardin, M. S. Katz, Y. Pei, H. L. Graber, M. B. Levin, R. D. Levina, N. A. Franco, W. B. Solomon, and R. L. Barbour, "Design and implementation of dynamic near-infrared optical 
tomographic imaging instrumentation for simultaneous dual-breast measurements," Appl. Opt. 44(11), 2140-2153 (2005).

237.A. A. Kokhanovsky, Light Scattering Media Optics: Problems and Solutions, Springer, Berlin (2004).

238. A. N. Tikhonov, and V. A. Arsenin, Solution of Ill-Posed Problems, Winston \& Sons, Washington (1977).

239.C. L. Lawson, and R. J. Hanson, Solving Least Squares Problems, Prentice-Hall, Englewood Cliffs (1974).

240.C. C. Paige, and M. A. Saunders, "LSQR: An algorithm for sparse linear equations and sparse least squares," ACM Trans. Math. Softw. 8, 43-71 (1982).

241.M. R. Hestenes, and E. Stiefel, "Methods of conjugate gradients for solving linear systems," J. Res. NBS. 49, 409-436 (1952).

242.L. Kaufman, "Maximum likelihood, least squares, and penalized least squares for PET," IEEE Trans. Med. Imag. 12, 200-214 (1993).

243.R. Gordon, R. Bender, and G. T. Herman, “Algebraic reconstruction techniques (ART) for three-dimensional electron microscopy and X-ray photography,” J. Theor. Biol. 29, 471-481 (1970).

244.C. L. Byrne, "Iterative image reconstruction algorithms based on cross-entropy minimization," IEEE Trans. Image Process. 2, 96-103 (1993).

245.D. W. Marquardt, “An algorithm for least-squares estimation of nonlinear parameters,” J. SIAM 11, 431-441 (1963).

246.M. Born, and E. Wolf, Principles of Optics, 7th ed., Cambridge University Press, Cambridge (1999).

247.S. M. Rytov, “Light diffraction on ultrasonic waves," Izv. Akad. Nauk SSSR, Ser. Fiz., No 2, $223-259$ (1937) [in Russian].

248. M. Suzen, A. Giannoula, and T. Durduran, "Compressed sensing in diffuse optical tomography," Opt. Express 18(23), 23676-23690 (2010).

249. O. Lee, J. M. Kim, Y. Bresler, and J. C. Ye, "Compressive diffuse optical tomography: noniterative exact reconstruction using joint sparsity," IEEE Trans. Med. Imag. 30(5), 1129-1142 (2011).

250.S. Okawa, Y. Hoshi, and Y. Yamada, "Improvement of image quality of time-domain diffuse optical tomography with $l_{\mathrm{p}}$ sparsity regularization," Biomed. Opt. Express 2(12), 3334-3348 (2011).

251. V. C. Kavuri, Z.-J. Lin, F. Tian, and H. Liu, "Sparsity enhanced spatial resolution and depth localization in diffuse optical tomography,” Biomed. Opt. Express 3(5), 943-957 (2012).

252.J. Prakash, C. Shaw, R. Manjappa, R. Kanhirodan, and P. K. Yalavarthy, "Sparse recovery methods hold promise for diffuse optical tomographic image reconstruction,” IEEE J. Sel. Top. Quantum Electron. 20(2), 6800609 (2014).

253.C. B. Shaw, and P. K. Yalavarthy, "Performance evaluation of typical approximation algorithms for nonconvex $l_{\mathrm{p}}$-minimization in diffuse optical tomography," J. Opt. Soc. Am. A 31(4), 852-862 (2014).

254.C. Chen, F. Tian, H. Liu, and J. Huang, "Diffuse optical tomography enhanced by clustered sparsity for functional brain imaging,” IEEE Trans. Med. Imag. 33(12), 2323-2331 (2014).

255.D. L. Donoho, “Compressed sensing,” IEEE Trans. Inf. Theory 52, 1289-1306 (2006).

256.E. J. Candes, J. K. Romberg, and T. Tao, "Stable signal recovery from incomplete and inaccurate measurements," Commun. Pure Appl. Math. 59, 1207-1223 (2006).

257.E. J. Candes, and J. K. Romberg, "Sparsity and incoherence in compressive sampling," Inverse Problems 23, 969-985 (2007).

258.J. Dutta, S. Ahn, C. Li, S. R. Cherry, and R. M. Leahy, "Joint $L_{1}$ and total variation regularization for fluorescence molecular tomography," Phys. Med. Biol. 57(6), 1459-1476 (2012).

259.Z. Xue, X. Ma, Q. Zhang, P. Wu, X. Yang, and J. Tian, “Adaptive regularized method based on homotopy for sparse fluorescence tomography,” Appl. Opt. 52(11), 2374-2384 (2013).

260.H. Yi, D. Chen, W. Li, S. Zhu, X. Wang, J. Liang, and J. Tian, "Reconstruction algorithms based on $l_{1}$-norm and $l_{2}$-norm for two imaging models of fluorescence molecular tomography: a comparative study," J. Biomed. Opt. 18(5), 056013 (2013).

261.J. Shi, B. Zhang, F. Liu, J. Luo, and J. Bai, "Efficient $L_{1}$ regularization-based reconstruction for fluorescent molecular tomography using restarted nonlinear conjugate gradient," Opt. Lett. 38(18), 3696-3699 (2013).

262.J. Ye, C. Chi, Z. Xue, P. Wu, Y. An, H. Xu, S. Zhang, and J. Tian, "Fast and robust reconstruction for fluorescence molecular tomography via a sparsity adaptive subspace pursuit method," Biomed. Opt. Express 5(2), 387-406 (2014).

263.D. Zhu, Y. Zhao, R. Baikejiang, Z. Yuan, and C. Li, “Comparison of regularization methods in fluorescence molecular tomography," Photonics 1, 95-109 (2014).

264.D. Zhu, and C. Li, "Nonconvex regularizations in fluorescence molecular tomography for sparsity enhancement,” Phys. Med. Biol. 59, 2901-2912 (2014).

265.A. Jin, B. Yazici, and V. Ntziachristos, "Light illumination and detection patterns for fluorescence diffuse optical tomography based on compressive sensing," IEEE Trans. Image Process. 23(6), 2609-2624 (2014).

266.W. Xie, Y. Deng, K. Wang, X. Yang, and Q. Luo, "Reweighted $L_{1}$ regularization for restraining artifacts in FMT reconstruction images with limited measurements," Opt. Lett. 39(14), 4148-4151 (2014). 
267.L. Zhao, H. Yang, W. Cong, G. Wang, and X. Intes, “ $L_{\mathrm{p}}$ regularization for early gate fluorescence molecular tomography," Opt. Lett. 39(14), 4156-4159 (2014).

268.F. Yang, M.S. Ozturk, L. Zhao, W. Cong, G. Wang, and X. Intes, "High-resolution mesoscopic fluorescence molecular tomography based on compressive sensing," IEEE Trans Biomed. Eng. 62, 248-255 (2015).

269.H. Guo, J. Yu, X. He, Y. Hou, F. Dong, and S. Zhang, "Improved sparse reconstruction for fluorescence molecular tomography with $L_{1 / 2}$ regularization," Biomed. Opt. Express 6(5), 1648-1664 (2015).

270.J. Shi, F. Liu, J. Zhang, J. Luo, and J. Bai, "Fluorescence molecular tomography reconstruction via discrete cosine transform-based regularization,” J. Biomed. Opt. 20(5), 055004 (2015).

271. Y. An, J. Liu, G. Zhang, J. Ye, Y. Du, Y. Mao, C. Chi, and J. Tian, “A novel region reconstruction method for fluorescence molecular tomography," IEEE Trans Biomed. Eng. 62, 1818-1826 (2015).

272.X. He, F. Dong, J. Yu, H. Guo, and Y. Hou, "Reconstruction algorithm for fluorescence molecular tomography using sorted $L$-one penalized estimation,” J. Opt. Soc. Am. A 32(11), 1928-1935 (2015).

273.S. Chen, D. Donoho, and M. Saunders, "Atomic decomposition by basis pursuit," SIAM J. Sci. Comput., 20(1), 33-61 (1999).

274.M. Schweiger, and S. R. Arridge, "The Toast++ software suite for forward and inverse modeling in optical tomography,” J. Biomed. Opt. 19(4), 040801 (2014).

275.H. Dehghani, M. E. Eames, P. K. Yalavarthy, S. C. Davis, S. Srinivasan, C. M. Carpenter, B. W. Pogue, and K. D. Paulsen, "Near infrared optical tomography using NIRFAST: Algorithm for numerical model and image reconstruction," Commun. Numer. Meth. Eng. 25(6), 711-732 (2009).

276. M. Jermyn, H. Ghadyani, M. A. Mastanduno, W. Turner, S. C. Davis, H. Dehghani, and B. W. Pogue, "Fast segmentation and high-quality three-dimensional volume mesh creation from medical images for diffuse optical tomography," J. Biomed. Opt. 18(8), 086007 (2013).

277.V. Y. Soloviev, K. B. Tahir, J. McGinty, D. S. Elson, M. A. Neil, P. M. French, and S. R. Arridge, "Fluorescence lifetime imaging by using time-gated data acquisition," Appl. Opt. 46, 7384-7391 (2007).

278.A. T. Kumar, S. B. Raymond, A. K. Dunn, B. J. Bacskai, and D. A. Boas, "A time domain fluorescence tomography system for small animal imaging," IEEE Trans. Med. Imag. 27, 1152-1163 (2008).

279.F. Gao, J. Li, L. Zhang, P. Poulet, H. Zhao, and Y. Yamada, "Simultaneous fluorescence yield and lifetime tomography from time-revolved transmittances of small-animal-sized phantom," Appl. Opt. 49, 3163-3172 (2010).

280.V. Venugopal, J. Chen, F. Lesage, and X. Intes, "Full-field time-resolved fluorescence tomography of small animals," Opt. Lett. 35, 3189-3191 (2010).

281.C. Darne, Y. Yujie, and E. M. Sevick-Muraca, "Small animal fluorescence and bioluminescence tomography: a review of approaches, algorithms and technology update," Phys. Med. Biol. 59, R1-R64 (2014).

282.V. V. Lyubimov, "Principles of fluorescence laser tomography of strongly scattering media," Optics and Spectroscopy 88(2), 282-285 (2000).

283.J. Hsieh, Computed Tomography: Principles, Design, Artifacts, and Recent Advances, SPIE Press, Bellingham, PM114 (2003).

284.S. P. Kotova, I. V. Mayorov, and A. M. Mayorova, "Application of neutral networks for determining optical parameters of strongly scattering media from the intensity profile of backscattered radiation," Quantum Electronics 37(1), 22-26 (2007).

285.B. A. Veksler, and I. V. Meglinski, “Application of the artificial neural network for reconstructing the internalstructure image of a random medium by spatial characteristics of backscattered optical radiation," Quantum Electronics 38(6), 576-579 (2008).

286.D. A. Boas, M. A. O’Leary, B. Chance, and A. G. Yodh, "Scattering of diffuse photon density waves by spherical inhomogeneities within turbid media: analytic solution and applications," Proc. Natl. Acad. Sci. USA. 91, 4887-4891 (1994).

287.M. S. Patterson, B. Chance, and B. C. Wilson, "Time resolved reflectance and transmittance for the noninvasive measurement of tissue optical properties," Appl. Opt. 28, 2331-2336 (1989).

\section{Contents}

1 Introduction

2 Structure and physiological properties of mammary gland in the norm and with neoplasms

3 Development of methods of measurement and visualisation of optical parameters

3.1 Diaphanography

3.2 Method of integrating spheres and breast optical parameters, determined using this method
3.3 Methods based on detection of diffusely scattered light

3.4 Diffuse optical tomography

4 Design problems and equipment of the diffuse optical mammotomograph

4.1 Design problems and operation principle of the diffuse optical mammotomograph

4.2 Techniques of tissue probing and optical signal detection 
4.3 Instrumentation for diffuse optical tomography 5 Methods of diffusion tomogram reconstruction

5.1 Methods based on inverting the operator equation

5.2 Perturbation semianalytic methods

5.3 Projection methods

5.4 Methods approximately formulating the inverse problem of diffuse optical tomography

6 Conclusion

\section{Introduction}

In the present-day practice of noninvasive and lowinvasive diagnostics of breast cancer, alongside with the traditional X-ray mammography, the new methods of medical imaging, such as X-ray computed tomography (X-ray CT), magnetic resonance tomography (MRT), methods of ultrasonic imaging (B-scan imaging, ultrasonic reflectivity tomography), methods of nuclear medicine (scintigraphy, positron emission tomography, and single-photon emission computed tomography) are used. However, the clinical efficiency of these methods is still not very high. One of the reasons is the absence of a relatively cheap method that could reliably differentiate malignant and benign tissues at different stages of the disease. The theoretical and experimental studies (see books [1-6], reviews [7-28], and papers [29-110]), as well as successful clinical tests $[1-4,6$, $13,17,18,20,22,23,25-28,59,61,63,77,81,100$, 101, 111-157] of recent years show that with high probability this gap can be filled by the rapidly progressing diffuse optical tomography (DOT) of strongly scattering (optically turbid) media. The diffusion tomography, like any other tomography, is aimed to answer the questions "What is there inside? What is the inner tissue structure? Are there any features that can be identified as pathological ones?" To answer these questions, the DOT technique reconstructs the spatial distributions of the tissue optical and functional parameters, basing on the results of spatially dependent measurements of the optical signal.

Similar to other kinds of tomography, DOT first solves the forward problem, i.e., seeks the characteristics of the radiation passed through the medium. Then the inverse problem is formulated and solved, i.e., the problem of determining and visualising the spatial distribution of the physical parameters of the medium itself. However, in contrast to the X-ray CT and the nuclear medicine methods, DOT makes use of the visible and near infrared (IR) radiation, harmless for humans in the so called therapeutic transparency window $(650-1200 \mathrm{~nm})$, where tissues possess the minimal level of absorption. DOT allows imaging with essentially higher contrast resolution than ultrasonic reflectivity tomography. As compared to MRT, DOT makes use of very simple and compact equipment and is a significantly cheaper method of medical imaging. In contrast to optical coherence tomography, successfully used in recent years for the diagnostics of optically transparent tissues of eye and surface layers of turbid tissues, DOT allows for the contribution to the recorded signal not only from ballistic photons that pass through the tissue without interaction, but also from the diffusion photons, experiencing multiple scattering. This property allows the examination of rather thick tissue samples (to 10-12 cm thick) if they do not contain bone structures. The mammary gland is just the suitable tissue. Therefore, the diagnostics of breast oncological diseases is the main potential clinical application of DOT. In this review, we present the state-of-the-art and prospects of breast DOT. In the literature, including the Russian one, for this method of medical imaging they also use the terms "diffuse optical reconstructive mammography" and "diffuse optical mammotomography". We will mainly use the latter of these terms.

The unique capability of DOT is that of separate reconstruction of the spatial distributions of different optical parameters of a tissue (first of all, the absorption coefficient and the reduced scattering coefficient) at different wavelengths. This feature makes it possible to visualise the concentrations of haemoglobin in oxygenated and deoxygenated forms and, therefore, to obtain the spatial pattern of the tissue oxygen saturation, as well as to visualise the fractional blood volume, the concentrations of cytochromes (bilirubin, melanin, and cytochrome oxidase), lipids and water. The visualisation of functional parameters, first of all, the haemoglobin concentration, the tissue oxygen saturation, and the fractional blood volume, is the main feature that determines the potential perspectives of DOT as a mammography method, since it offers a possibility of spatial localisation of such phenomenon as vascularisation of cancer tissues and, hence, of breast oncologic disease diagnostics.

At present many universities, research centres and large commercial companies perform intense studies in the field of DOT aimed at the entry to the consumer market. The main obstacle for wider use of DOT in everyday practice of medical diagnostics is the lack of fast reconstruction algorithms that could provide diffusion optical tomograms in real time with acceptable accuracy. The problem is that due to multiple scattering, the photons have no regular trajectories and are distributed over the entire studied volume. As a result, every point of the volume makes an essential contribution to the recorded signal, which causes strong nonlinearity of both the forward and the inverse DOT problems. From the mathematical point of view, the solution of the inverse problem implies the inversion of a nonlinear operator equation. Therefore, for reconstructing the spatial distributions of optical parameters they commonly use multistep algorithms [3, $7,12,21,24,29,33,37,40-42,45,48,50,55,59-62$, $68,70,73,76,77,88,91,103,116,119,123-125]$, based on step-by-step linearization of the reconstruction problem and multiple inversion of the system of linear algebraic equations that describe the discrete model. Although these algorithms are considered as the most precise DOT algorithms, they do not allow reliable resolution of objects smaller than $4 \mathrm{~mm}$. Just this fact 
causes the doubt in the potential possibility of using DOT for cancer diagnostics at the very early stages of the tumour development, since only the cancer tumours smaller than 2-4 $\mathrm{mm}$ have real possibility to be noninvasive [158]. Moreover, the multistep algorithms take tens of minutes or even hours to get a 3D image, which is also not quite satisfactory for modern requirements of medical diagnostics. Thus, the increase of the reconstruction accuracy and the reduction of the processing time are two goals pursued now by the researchers, developing the methods for diffusion tomogram reconstruction.

The simplest way to reduce the time of data processing is to use the perturbation model of reconstruction that neglects the nonlinearity of the inverse problem, reduces its solution to the inversion of a linear integral Fredholm equation of the first kind, and allows one to replace the multistep reconstruction procedure with a single-step one. The perturbation model is considered as particularly applicable in the optical mammotomography, when the objects to be reconstructed and spatially localised are small optical inhomogeneities (cancer tumours) against the background of a "quasihomogeneous" scattering medium (healthy fibrous-glandular tissue). In recent years the collaboration of researchers from the Vavilov State Optical Institute Corporation (SOI, SaintPetersburg) and the Russian Federal Nuclear Center Zababakhin Institute of Applied Physics (RFNCVNIITF, Snezhinsk) [5, 35, 36, 43, 46, 47, 57, 59, 66, $75,79,80,83,84,93,98,104,106,110]$ developed theoretically an original perturbation approach to the solution of the inverse DOT problem, based on the probabilistic interpretation of the transfer of light energy by photons from the source to the detector. The proposed approach not only reduces the processing time to seconds and parts of a second [5, 57], but also is able to compete successfully with the multistep algorithms in the precision of reconstruction. Thus, the recent studies by the RFNC-VNIITF researchers [104, 106] demonstrated, yet in the numerical experiment, the resolution of an inhomogeneity smaller than $3 \mathrm{~mm}$ within the object with the size of $8 \mathrm{~cm}$, which is recordbreaking for diffusion tomography.

High-quality diffusion tomograms using the own original reconstruction methods are obtained also by the Russian researchers from the Biophotonics Laboratory, Institute of Applied Physics of the Russian Academy of Sciences (IAP RAS, Nizhny Novgorod) [143, 159-161] and the International Laser Center, Lomonosov Moscow State University (MSU, Moscow) [44, 53, 54, 58, 105]. The approximate DOT methods, such as the methods of optical inhomogeneity localisation, were developed by the team from the Biomedical Engineering Chair, Tambov State Technical University (TSTU, Tambov) [92, 99, 102, 109]. Each of these Russian teams has its experimental base and tests its original DOT methods using phantoms and laboratory animals.

Acknowledging the achievements of Russian scientists in DOT, we should mention also the development of methods for in vitro and in vivo measurement of tissue optical parameters [1, 162-171]. From the point of view of DOT methodological base, the knowledge of optical parameters is necessary for constructing adequate spatial optical models of tissues and for testing the methods of diffusion tomogram reconstruction at the stages of numerical and physical experiments, as well as in clinical testing.

In the present review, we briefly characterise the achievements of each of the above teams of Russian researchers. The paper is organised as follows. Section 1 is devoted to the structure and physiological properties of the mammary gland in the normal condition and in the presence of neoplasms. Section 2 presents the methods of measurement and visualisation of optical parameters, the development of which was the main premise for the present-day DOT success. In Section 3, the main problems of designing the diffusion mammotomograph are formulated and the time-domain DOT instrumentation is briefly described. Finally, in Section 4 the methods of solving the inverse DOT problem are considered, with the emphasis on the methods developed by Russian research teams. The conclusions about the state-of-the-art and the prospects of diffusion mammotomography development are formulated in the end of the paper.

\section{Structure and physiological properties of mammary gland in the norm and with neoplasms}

The mammary gland is located in the subcutaneous layer of the thoracic cage. It occupies the gap between the sternum edge and the anterior axillary line at the level of III-VI (VII) ribs, in front of the greater pectoral muscle and partially the serratus anterior muscle, covered with fascia pectoralis (Fig. 1) [172]. The shape and size of the gland in women depends upon the sexual development and individual features. The skin over the mammary gland is thin and stiff in relation to the gland, mainly because the subcutaneous fatty tissue of the breast is penetrated by connective tissue intersections connecting the skin with the gland capsule. Near the nipple and areole, there is no subcutaneous fat. The mammary gland is surrounded by the capsule formed by the leaves of the superficial fascia. Between the upper edge of the gland and the collarbone, the fascia is thickened and 1-3 ligaments can be distinguished in it, which suspend the mammary gland [173, 174].

The basic components of a mature mammary gland are the alveoli (hollow cavities, a few millimetres large) lined with milk-secreting cuboidal cells and surrounded by myoepithelial cells. These alveoli join to form groups known as lobules [175]. From the fascial capsule of the mammary gland multiple connective tissue branches penetrate into its depth and surround 15-20 lobules of the mammary gland as such, having lactiferous ducts with the diameter of $2-3 \mathrm{~mm}$. The ducts radially converge towards the nipple, near the base of which they become wider forming the 
lactiferous sinuses. In the nipple, the lactiferous ducts become narrow again and merge by $2-3$ to end via $8-15$ point openings at the top of the nipple. Behind the mammary gland capsule between it and fascia propria, covering the greater pectoral muscle, there is a layer of loose cellular tissue referred to as retromammary tissue. The looseness of this tissue provides the mobility of the mammary gland with respect to the chest wall [173, 174].

Multiple branches of blood vessels implement the blood supply of the mammary gland. Deep veins accompany the arteries of the same name, and the surface ones form a subcutaneous network, connected with the axillary vein and other branches of subcutaneous vein network. The chains of lymph nodes accompany the arteries [174].

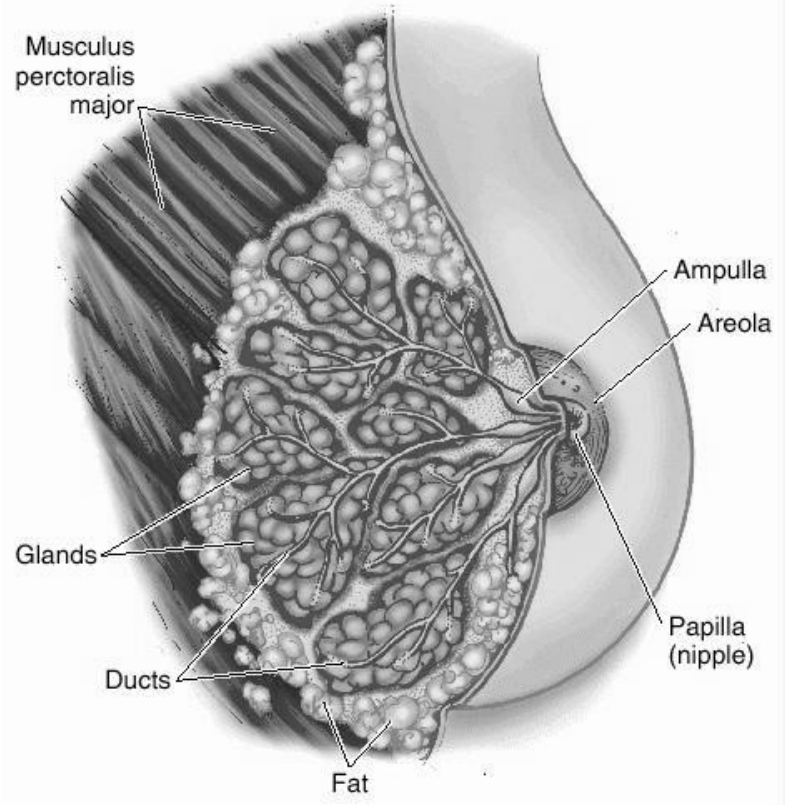

Fig. 1 Schematic of breast [172].

The lymphatic system of the mammary gland is well developed and closely connected with the adjacent lymphatic basins. The surface network of lymphatic capillaries is connected with the skin lymphatic network; the deep network begins from the intralobular and interlobular lymphatic capillaries and anastomoses with the surface skin lymphatic vessels. From the deep network of the mammary gland, the larger deferent lymphatic vessels take origin [173, 174]. The central nodes serve as collectors for all vessels that carry the lymph to the axilla, and are most frequently affected by the breast cancer [174]. The relative frequency of remote metastases of the breast cancer, particularly, in lungs and bones, is explained by early penetration of tumour elements from the lymphatic bed to the blood circulation one, even before the expressed metastases appear in the regional lymphatic nodes [174].
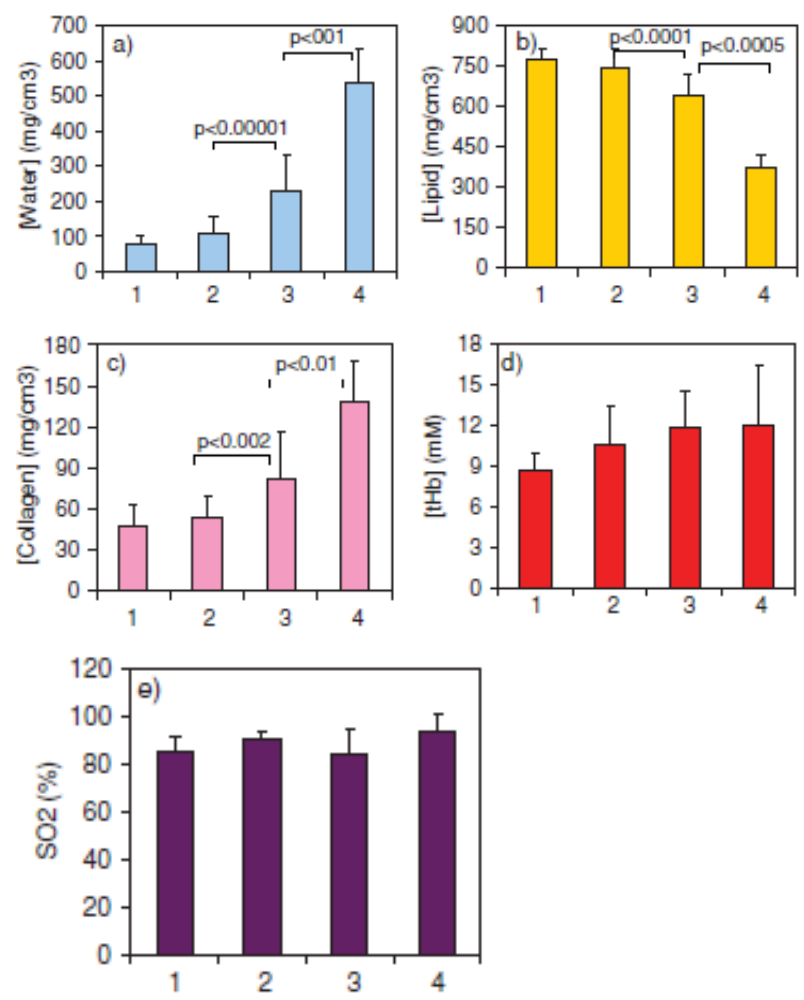

Fig. 2 Content of the main components of breast tissue depending on the type: (a) water, (b) lipids, (c) collagen, (d) haemoglobin $(t H b)$, (e) haemoglobin oxygen saturation $\left(\mathrm{SO}_{2}\right)$. The results are presented in the form "mean value \pm standard deviation". The difference between the groups was considered significant if $\mathrm{p}<0.05$. The abscissa shows the mammographic density of the breast (from 1st to 4th type). The analysis involved 49 volunteers [178].

Four types of breasts are distinguished by their density [176]: 1) the breasts with predominant fat component, the presence of heterogeneous regions of fibrous-glandular tissue occupying less than $25 \%$ of the mammogram area; 2) the regions of fibrous-glandular tissue occupy from 25 to $50 \%$ of the mammogram area; 3 ) breasts with the area of fibrous-glandular tissue from 51 to $75 \%$ of the mammogram area, and 4) very dense breasts with more than $75 \%$ of the mammogram area occupied by the fibrous-glandular tissue [177]. The content of the basic components (water, lipids, collagen, haemoglobin) and the oxygen saturation of haemoglobin vary depending on the tissue density of mammary glands. Thus, the authors of Ref. [178] have shown that the content of water in the tissue considerably grows with increasing density and, upon the average, varies from $\sim 75 \mathrm{mg} / \mathrm{cm}^{3}$ (for the $1^{\text {st }}$ type) to $\sim 540 \mathrm{mg} / \mathrm{cm}^{3}$ (for the $4^{\text {th }}$ type) (Fig. 2 (a)). This is accompanied by the appropriate reduction of lipid content: upon the average, from $\sim 760 \mathrm{mg} / \mathrm{cm}^{3}$ (for the $1^{\text {st }}$ type) to $325 \mathrm{mg} / \mathrm{cm}^{3}$ (for the $4^{\text {th }}$ type) (Fig. 2 (b)). The amount of collagen, associated with the content of the fibrous-glandular tissue, also grows with the increase of the tissue density: from $\sim 45 \mathrm{mg} / \mathrm{cm}^{3}$ (for the $1^{\text {st }}$ type) to $\sim 135 \mathrm{mg} / \mathrm{cm}^{3}$ (for the $4^{\text {th }}$ type) (Fig. 2 (c)). Compared to the tissue with 
predominant fat component, the dense fibrous-glandular one must possess enhanced vascularisation and, therefore, increased concentration of haemoglobin $(t H b)$, which is observed in Fig. 2 (d). However, the difference in haemoglobin concentration in different types of breast tissues is not statistically significant $(\sim 8$ and $\sim 12 \mu \mathrm{M}$ for the $1^{\text {st }}$ and the $4^{\text {th }}$ type, respectively). The oxygen saturation ( $\mathrm{SO}_{2}$ ) manifests no tendency depending on the tissue density (the mean value for all types of tissues amounts to $99 \%$ ) (Fig. 2 (e)).

In Ref. [179], as a result of averaging over 5 patients, the following mean values of physiological parameters of healthy breast were found: the content of water $18.7 \pm 10.3 \%$, the content of lipids $66.1 \pm 10.3 \%$, the concentration of total haemoglobin $17.5 \pm 7.5 \mu \mathrm{M}$, the concentration of haemoglobin in oxygenated and deoxygenated forms $12.4 \pm 6.2 \mu \mathrm{M}$ and $5.10 \pm 1.7 \mu \mathrm{M}$, respectively, the oxygen saturation $67.7 \pm 9.3 \%$.

Fibrous-cystous neoplasms and fibroadenoma are the most common benign tumours in the breast tissues. In the case of fibrous-cystous neoplasms, one observes the proliferation of the connective tissue with predomination of fibrous structure. The fibroadenoma is characterised by the dense well-shaped capsule and dense elastic consistence. The heterologous fibroadenoma possesses soft elastic consistence and tendency to progressive growth. In Ref. [180] it was shown that the epithelium-to-stroma ratio in the fibroadenoma amounts to 0.27 , which means the growth of stroma as a characteristic feature of this neoplasm.

Histologically the following types of breast cancer tumours are distinguished: ductal carcinoma in situ, lobular carcinoma in situ, invasive (infiltrating) ductal carcinoma, invasive (infiltrating) lobular carcinoma, inflammatory breast cancer, tubular carcinoma, medullary carcinoma, colloid (mucin-producing, signet ring cell) carcinoma, papillary carcinoma, metaplastic cancer (squamous-cell carcinoma), cancer with osteoclast-like cells, adenoid-cystous cancer, secretory carcinoma (juvenile cancer), cystous hypersecretory carcinoma, apocrine cancer, cancer with signs of endocrine neoplasm (primary carcinoid tumour, apudoma), cribrous cancer [181].

The aetiology of breast cancer is subject to multiple factors. In particular, the neoplasm development can be provoked by early menarche, late menopause, burdened familiar history, obesity, diabetes mellitus, using exogenous hormones for contraception or treatment, etc. [175]. Almost all cases of breast cancer occur in the lobules or ducts of the mammary glands. The frequent ductal carcinoma in situ and invasive ductal carcinoma are characterised by the increased cellularity and enlarged nuclei depending on the stage [182]. According to Ref. [118] the epithelium-to-stroma ratio for the invasive ductal carcinoma amounts to 1.41, which means the predomination of malignant epithelium. The colloid carcinoma is a relatively rare disease, in which the cancer cells secrete extracellular mucin, thus providing the transparent component of the tissue, in which the cancer cells are merged [180].
A characteristic feature of benign and malignant neoplasms is the enhancement of their blood supply as compared to the healthy tissue. In Ref. [118] it is shown that in the sample of a fibroadenoma the total density of blood vessels per unit area amounts to $2.27 \%$, and in the centre of the mass this value is somewhat lower $(1.92 \%)$, than in the periphery $(2.62 \%)$. The haemoglobin concentration peak in the neoplasm amounts to $55 \mu \mathrm{M}(3.66 \mathrm{~g} / \mathrm{L})$, the mean concentration in the surrounding normal tissue being $33 \pm 5 \mu \mathrm{M}(2.2 \pm 0.3$ $\mathrm{g} / \mathrm{L})$.

For a sample of invasive ductal carcinoma, the characteristic value of the total density of blood vessels per unit area is $5.07 \%$ with the decrease in the centre $(4.22 \%)$, as compared to the periphery of the neoplasm $(5.91 \%)$. The peak of haemoglobin concentration in the neoplasm amounts to $68 \mu \mathrm{M}(4.5 \mathrm{~g} / \mathrm{L})$, the mean concentration in the surrounding healthy tissue being 30 $\pm 6 \mu \mathrm{M}(2.0 \pm 0.4 \mathrm{~g} / \mathrm{L})[118]$.

Ref. [179] presents the results of averaging of the physiological parameters of 58 malignant tumours of breast. In this group the water content was $25.9 \pm 13.5 \%$; the content of lipids was $58.5 \pm 14.3 \%$; the total haemoglobin concentration was $24.7 \pm 9.8 \mu \mathrm{M}$; the concentrations of haemoglobin in oxygenated and deoxygenated forms were $17.2 \pm 7.5 \mu \mathrm{M}$ and $7.53 \pm 2.8$ $\mu \mathrm{M}$, respectively; the oxygen saturation was $67.5 \pm 8.4 \%$.

\section{Development of methods of measurement and visualisation of optical parameters}

\subsection{Diaphanography}

Although the optical mammography is still in the making, this method of medical visualisation has deep historical roots. The first clinical results of optical breast transillumination with $\mathrm{CW}$ radiation were reported by Culter in 1929 [183]. The object of study was placed between the source (a xenon lamp) and the doctor, and the shadow "images", characterising the difference between the healthy and pathologic tissues were observed visually. The "images" were too blurred, and the attempts to improve the contrast by increasing the intensity lead to overheating the skin surface. Gross, who called the method "diaphanography", published the first report in 1972 about the real possibility of differentiating benign and malignant tumours in this way [184]. A certain improvement of contrast was achieved by recording the breast image with a photo camera with colour IR film [185] or a TV camera with video recorder [186]. However, the resolution of the images was limited by $2 \mathrm{~cm}$. In 1990 the group of Swedish researchers published the data [187], according to which the probability of detecting small tumours using the method of diaphanography was estimated as negligibly small, and the probability of erroneous diagnosis as exceeding by three times the error probability of traditional methods, such as X-ray mammography and B-scan imaging. This paper and some other publications made doubtful the prospects of 
diaphanography as a method of early breast cancer diagnostics and decreased the interest to the visualisation of breast using visible and near IR radiation.

\subsection{Method of integrating spheres and breast optical parameters, determined using this method}

Starting from the middle of the 20-th century, the methods of determining the optical parameters of tissue samples in vitro have been developed rapidly. In this field the main stages were associated with the spectrophotometry using integrating spheres [1, 164, $180,188-192]$ and the development of iteration methods, such as inverse adding-doubling method [180, 192, 193], and the inverse Monte Carlo method [190]. Among the methods of determining the optical parameters of tissues, the most widespread one is the method of integrating spheres. According to this method, the setup incorporating, besides the source and detectors, one or two integrating spheres is used to measure such parameters, as the collimated transmission, total transmission, and diffuse reflection. To determine the local optical parameters of the tissues, i.e., the absorption coefficient $\mu_{a}$, the reduced scattering coefficient $\mu_{s}^{\prime}$, and the anisotropy factor $g$, the inverse models and methods are used, based on the radiative transport theory, e.g., the two-flux KubelkaMunk model [1, 162, 164, 188]. Below we present some results of measuring the breast optical parameters by means of the method of integrating spheres.

As shown in Ref. [180], in the norm the absorption coefficient of the breast fibrous tissue equals $0.127 \pm 0.187 \mathrm{~cm}^{-1}(\lambda=749 \mathrm{~nm}), 0.06 \pm 0.12 \mathrm{~cm}^{-1}(\lambda=$ $789 \mathrm{~nm})$, and $0.05 \pm 0.03 \mathrm{~cm}^{-1}(\lambda=836 \mathrm{~nm})$, for the breast adipose tissue the absorption coefficient equals $0.184 \pm 0.159 \mathrm{~cm}^{-1}(\lambda=749 \mathrm{~nm}), 0.08 \pm 0.105 \mathrm{~cm}^{-1}(\lambda=$ $789 \mathrm{~nm})$, and $0.108 \pm 0.097 \mathrm{~cm}^{-1}(\lambda=836 \mathrm{~nm})$. For the infiltrating carcinoma at these wavelengths the values of the absorption coefficient amount to $0.147 \pm 0.144 \mathrm{~cm}^{-1}$, $0.044 \pm 0.083 \mathrm{~cm}^{-1}$, and $0.1 \pm 0.188 \mathrm{~cm}^{-1}$, respectively. For the mucinous carcinoma the values of the absorption coefficient are equal to $0.259 \pm 0.198 \mathrm{~cm}^{-1}, 0.016 \pm 0.072$ $\mathrm{cm}^{-1}$, and $0.024 \pm 0.108 \mathrm{~cm}^{-1}$, respectively, and for the ductal carcinoma the absorption coefficient values amount to $0.076 \pm 0.068 \mathrm{~cm}^{-1}, 0.023 \pm 0.034 \mathrm{~cm}^{-1}$ and $0.039 \pm 0.068 \mathrm{~cm}^{-1}$, respectively.

The reduced scattering coefficient of the breast fibrous tissue equals $9.75 \pm 2.27 \mathrm{~cm}^{-1}(\lambda=749 \mathrm{~nm})$, $8.95 \pm 2.45 \mathrm{~cm}^{-1}(\lambda=789 \mathrm{~nm})$, and $8.1 \pm 2.21 \mathrm{~cm}^{-1}(\lambda=$ $836 \mathrm{~nm}$ ), for the adipose tissue the reduced scattering coefficient equals $8.48 \pm 3.43 \mathrm{~cm}^{-1}(\lambda=749 \mathrm{~nm})$, $7.67 \pm 2.57 \mathrm{~cm}^{-1}(\lambda=789 \mathrm{~nm})$, and $7.27 \pm 2.4 \mathrm{~cm}^{-1}(\lambda=$ $836 \mathrm{~nm})$. For the infiltrating carcinoma at these wavelengths the values of the reduced scattering coefficient amount to $10.91 \pm 5.59 \mathrm{~cm}^{-1}, 10.13 \pm 5.05 \mathrm{~cm}^{-}$ ${ }^{1}$, and $9.1 \pm 4.54 \mathrm{~cm}^{-1}$, respectively. For the mucinous carcinoma the values of the reduced scattering coefficient are equal to $6.15 \pm 2.44 \mathrm{~cm}^{-1}, 5.09 \pm 2.42 \mathrm{~cm}^{-1}$, and $4.78 \pm 3.67 \mathrm{~cm}^{-1}$, respectively, and for the ductal carcinoma the values of $\mu_{s}^{\prime}$ amount to $13.11 \pm 2.85 \mathrm{~cm}^{-1}$, $12.21 \pm 2.45 \mathrm{~cm}^{-1}$, and $10.46 \pm 2.65 \mathrm{~cm}^{-1}$, respectively [180].

As clearly seen from the presented data, neither the absorption coefficient, nor the reduced scattering coefficient measured at the above wavelengths (749, 789 и $836 \mathrm{~nm}$ ) in vitro allow reliable differentiation of healthy and pathologically modified tissues.

The similar conclusion follows from the analysis of the results, presented in Ref. [190], where in the spectral region 500-1100 $\mathrm{nm}$ the homogenised samples of breast tissues were studied, namely, the normal glandular breast tissue, the normal breast adipose tissue, the fibrocystic tissue, the fibroadenoma, and the ductal carcinoma. The authors have shown that at the wavelengths 540, 700, and $900 \mathrm{~nm}$ the values of the absorption coefficient are the following: for the normal glandular breast tissue $3.58 \pm 1.56 \mathrm{~cm}^{-1}(\lambda=540 \mathrm{~nm})$, $0.47 \pm 0.11 \mathrm{~cm}^{-1}(\lambda=700 \mathrm{~nm})$, and $0.62 \pm 0.05 \mathrm{~cm}^{-1}(\lambda=$ $900 \mathrm{~nm})$; for the normal breast adipose tissue $2.27 \pm 0.57$ $\mathrm{cm}^{-1}(\lambda=540 \mathrm{~nm}), 0.7 \pm 0.08 \mathrm{~cm}^{-1}(\lambda=700 \mathrm{~nm})$, and $0.75 \pm 0.08 \mathrm{~cm}^{-1}(\lambda=900 \mathrm{~nm})$; for the fibrocystic tissue $1.64 \pm 0.66 \mathrm{~cm}^{-1}(\lambda=540 \mathrm{~nm}), 0.22 \pm 0.09 \mathrm{~cm}^{-1}(\lambda=700$ $\mathrm{nm})$, and $0.27 \pm 0.11 \mathrm{~cm}^{-1}(\lambda=900 \mathrm{~nm})$; for the fibroadenoma the values of $\mu_{a}$ are $4.38 \pm 3.14 \mathrm{~cm}^{-1}(\lambda=$ $540 \mathrm{~nm}), 0.52 \pm 0.47 \mathrm{~cm}^{-1}(\lambda=700 \mathrm{~nm})$, and $0.72 \pm 0.53$ $\mathrm{cm}^{-1}(\lambda=900 \mathrm{~nm})$, and for the carcinoma $3.07 \pm 0.99 \mathrm{~cm}^{-}$ ${ }^{1}(\lambda=540 \mathrm{~nm}), 0.45 \pm 0.12 \mathrm{~cm}^{-1}(\lambda=700 \mathrm{~nm})$, and $0.5 \pm 0.15 \mathrm{~cm}^{-1}(\lambda=900 \mathrm{~nm})$.

The values of the reduced scattering coefficient are the following: for the normal glandular breast tissue $24.4 \pm 5.8 \mathrm{~cm}^{-1}(\lambda=540 \mathrm{~nm}), 14.2 \pm 3.0 \mathrm{~cm}^{-1}(\lambda=700$ $\mathrm{nm})$, and $9.9 \pm 2.0 \mathrm{~cm}^{-1}(\lambda=900 \mathrm{~nm})$; for the normal breast adipose tissue $10.3 \pm 1.9 \mathrm{~cm}^{-1}(\lambda=540 \mathrm{~nm})$, $8.6 \pm 1.3 \mathrm{~cm}^{-1}(\lambda=700 \mathrm{~nm})$, and $7.9 \pm 1.1 \mathrm{~cm}^{-1}(\lambda=900$ $\mathrm{nm})$; for the fibrocystic tissue, $21.7 \pm 3.3 \mathrm{~cm}^{-1}(\lambda=540$ $\mathrm{nm}), 13.4 \pm 1.9 \mathrm{~cm}^{-1}(\lambda=700 \mathrm{~nm})$, and $9.5 \pm 1.7 \mathrm{~cm}^{-1}(\lambda=$ $900 \mathrm{~nm})$; for the fibroadenoma $11.1 \pm 3.0 \mathrm{~cm}^{-1}(\lambda=540$ $\mathrm{nm}), 7.2 \pm 1.7 \mathrm{~cm}^{-1}(\lambda=700 \mathrm{~nm})$, and $5.3 \pm 1.4 \mathrm{~cm}^{-1}(\lambda=$ $900 \mathrm{~nm})$, and for the carcinoma $19.0 \pm 5.1 \mathrm{~cm}^{-1}(\lambda=540$ $\mathrm{nm}), 11.8 \pm 3.1 \mathrm{~cm}^{-1}(\lambda=700 \mathrm{~nm})$, and $8.9 \pm 2.6 \mathrm{~cm}^{-1}(\lambda=$ $900 \mathrm{~nm})$.

Thus, we can conclude that the use of the integrating sphere technique in the visible range of wavelengths (up to $1 \mu \mathrm{m}$ ) does not allow reliable differentiation of healthy and pathologically modified tissues. However, as shown in Ref. [192], the measurements carried out in the near IR spectral range, in the region of absorption bands of water $\left(\lambda_{\max } \sim 1450 \mathrm{~nm}\right)$ and lipids $\left(\lambda_{\max } \sim 1720\right.$ $\mathrm{nm})$ allow reliable differentiation of normal tissue, benign and malignant neoplasms. Thus, at the wavelength $1450 \mathrm{~nm}$ the absorption coefficient of the normal tissue amounts to $0.6686 \pm 0.0418 \mathrm{~mm}^{-1}$, for benign neoplasms $\mu_{a}=1.3402 \pm 0.0912 \mathrm{~mm}^{-1}$, while for the malignant neoplasms $\mu_{a}=1.8864 \pm 0.1272 \mathrm{~mm}^{-1}$, 
which is a sign of increasing content of water in the tumour in the course of its development. At the same time, with the development of the tumours the reduction of the content of lipids is observed, which is seen from the decrease of the absorption coefficient at the wavelength $1720 \mathrm{~nm}$ from $0.8766 \pm 0.0308 \mathrm{~mm}^{-1}$ to $0.6208 \pm 0.0336 \mathrm{~mm}^{-1}$. It is interesting to note, that, when passing from the normal tissue to the pathologically modified one, the reduced scattering coefficient in the wavelength range from 400 to 1000 $\mathrm{nm}$ also essentially (nearly by $2-2.5$ times) decreases. In this case, one can clearly see the increasing contribution of sufficiently small scatterers to the formation of the scattering spectrum, which is a manifestation of destruction of structure elements of the breast tissue with the development of the neoplasm. In the spectral range from 1000 to $2200 \mathrm{~nm}$, no essential change in the light scattering is observed [192].

\subsection{Methods based on the detection of diffusely scattered light}

The theoretical achievements of 70-90s of the last century (the models of photon migration, the discovery of diffuse photon density waves, the development of the dynamic light scattering theory, etc.), the progress of laser facilities and fibre optics, as well as the development of time-resolved techniques of tissue irradiation and signal detection, facilitated the intense development of optical methods for measurements in vivo, based on detecting the diffusely scattered light. These methods include, first, the pulse-oximetry [194, 195], the diffusing wave spectroscopy $[1,15,20,22,27$, $79,196,197]$, and the near IR spectroscopy [1-4, 10, 19, 20, 22, 25, 31, 85, 113, 116, 121, 132, 179, 198-214].

The pulse oximetry is grounded on measuring the intensity change of light transmitted through the tissue, synchronous in time with the heartbeat. After each contraction the arteries become wider, increasing the relative volume of blood in the tissue, and therefore, increasing the value of the absorption coefficient. By recording the difference between the maximal and minimal absorption, one can indirectly determine the degree of arterial blood oxygenation.

The diffusing wave spectroscopy is based on the measurements of temporal correlations of spatiotemporal fluctuations of light fields in a strongly scattering medium. The method allows quantitative characterisation of the motion of scattering particles in tissues and, therefore, can be used to measure the blood flow velocity [215, 216]. In Refs. [216-218] it was shown that the diffusion propagation of temporal correlations in a randomly inhomogeneous medium, consisting of different spatially separated scattering regions, is sensitive to the dynamics of the scatterer motion in these regions. In principle, this fact allows one to use position-dependent measurements of the temporal autocorrelation function of the field for tomographic reconstruction of the images of the dynamical inhomogeneities in the medium. This approach, referred to as diffuse optical correlation tomography in the literature $[15,20,22,27,79,219$ 223], has undoubtful prospects and is extensively developed now.

In contrast to the pulse oximetry, the near IR spectroscopy (or diffuse optical spectroscopy) allows the determination of the haemoglobin oxygen saturation not only in the arteries, but also in the tissue as a whole, including veins and capillaries. The near IR spectroscopy is based on irradiating the tissue with laser radiation at different wavelengths within the therapeutic transparency window and recording the optical signal using the frequency-domain, time-domain or continuous-wave spectroscopy technique (see Section 3.2). Both the transmitted light (transmission or transillumination spectroscopy) and the diffusely reflected radiation (reflectance or backscattering spectroscopy) are detected. The optical parameters $\mu_{a}$ and $\mu_{s}^{\prime}$ are determined indirectly from the results of the performed measurements. As clearly seen from the presented list of references $[1-4,10,19,20,22,25,31$, $85,113,116,121,132,179,198-214]$, the frequencydomain technique $[85,113,202]$ and the time-domain technique $[85,121,119,198,201,206-209,212]$ of transmission spectroscopy are most frequently used. The reflectance spectroscopy is also often used for in vivo measurements of the breast optical parameters, both in the frequency-domain $[25,179,199,200,205$, 209] and in the time-domain [25, 201, 214] and continuous-wave $[179,199,200,203,204,211]$ techniques. The near IR spectroscopy makes use of the same principles and the same methods of data recording, as the modern optical imaging methods, based on the detection of diffusely scattered light, and in this sense is their direct predecessor.

Table 1 summarises the optical parameters of different breast tissues measured in vivo, both in the normal condition and in the presence of benign and malignant neoplasms. From the Table it is clearly seen that most of the optical characteristics are studied in the first transparency window. At the same time, essential progress of laser technology and detection facilities allows one to broaden the used range of wavelengths and proceed to the second $(1600-1800 \mathrm{~nm})$ and the third (2100-2300 nm) transparency windows. This will surely yield new (may be crucial) information that can allow further advance of the methods of optical diagnostics towards the next principally new level and extend the capabilities of modern medicine in fighting the breast cancer.

The method of indirect determination of the functional parameters, such as the tissue oxygen saturation $\mathrm{SO}_{2}$ and the fractional blood volume $\delta \mathrm{V}$, is based on the difference in the absorption spectra of haemoglobin in the oxygenated $\mathrm{HbO}_{2}$ and deoxygenated $H b$ forms, as well as the known relation for the total absorption coefficient of a tissue at the wavelength $\lambda$ : 


$$
\mu_{a}(\lambda)=\sum_{n} \varepsilon_{n}(\lambda) C_{n}
$$

where $\varepsilon_{n}(\lambda)$ is the specific absorption coefficient, and $C_{n}$ is the concentration of the $n$-th chromophore in the tissue. Assume that we managed to determine the absorption coefficients $\mu_{a}\left(\lambda_{1}\right)=\mu_{a}^{1}$ and $\mu_{a}\left(\lambda_{2}\right)=\mu_{a}^{2}$ of the breast tissue at two different wavelengths $\lambda_{1}$ and $\lambda_{2}$ . In order to find $\mathrm{SO}_{2}$ and $\delta \mathrm{V}$, it is necessary to assess the absorption spectrum of the completely bloodless tissue $\mu_{a}^{b g}(\lambda)$ and also to know the volume fraction of erythrocytes (haematocrit) $C_{g}$ in the blood. For example, assuming that $\mu_{a}^{b g}\left(\lambda_{1}\right) \approx \mu_{a}^{b g}\left(\lambda_{2}\right) \approx \mu_{a}^{b g}$ the following relations can be derived [224]

$$
\begin{aligned}
& S O_{2}=\frac{C_{H b O_{2}}}{C_{H b O_{2}}+C_{H b}}= \\
& =\frac{\left(\mu_{a}^{1}-\mu_{a}^{2}+\mathrm{E}_{H b}^{2}-\mathrm{E}_{H b}^{1}\right) \mu_{a}^{b g}+\mathrm{E}_{H b}^{1} \mu_{a}^{2}-\mathrm{E}_{H b}^{2} \mu_{a}^{1}}{\left(\mathrm{E}_{H b O_{2}}^{2}-\mathrm{E}_{H b}^{2}\right)\left(\mu_{a}^{1}-\mu_{a}^{b g}\right)+\left(\mathrm{E}_{H b O_{2}}^{1}-\mathrm{E}_{H b}^{1}\right)\left(\mu_{a}^{2}-\mu_{a}^{b g}\right)},
\end{aligned}
$$

$$
\begin{aligned}
& \delta V=\frac{\text { blood volume in tissue }}{\text { tissue volume }}=1+ \\
& +\frac{\mathrm{E}_{H b}^{2} \mathrm{E}_{H b O_{2}}^{1}-\mathrm{E}_{H b}^{1} \mathrm{E}_{H b O_{2}}^{2}+\mu_{a}^{1}\left(\mathrm{E}_{H b O_{2}}^{2}-\mathrm{E}_{H b}^{2}\right)-\mu_{a}^{2}\left(\mathrm{E}_{H b O_{2}}^{1}-\mathrm{E}_{H b}^{1}\right)}{\mathrm{E}_{H b}^{1} \mathrm{E}_{H b O_{2}}^{2}-\mathrm{E}_{H b}^{2} \mathrm{E}_{H b O_{2}}^{1}+\mu_{a}^{b g}\left(\mathrm{E}_{H b}^{2}-\mathrm{E}_{H b}^{1}+\mathrm{E}_{H b O_{2}}^{1}-\mathrm{E}_{H b O_{2}}^{2}\right)} .
\end{aligned}
$$

Here $\mathrm{E}_{\mathrm{Hb} \mathrm{O}_{2}}^{1}, \mathrm{E}_{\mathrm{Hb}}^{1}, \mathrm{E}_{\mathrm{HbO}}^{2}, \mathrm{E}_{\mathrm{Hb}}^{2}$ are the reduced specific absorption coefficients of the chromophores $\mathrm{HbO}_{2}$ and $H b$, found by multiplying the specific coefficients $\varepsilon_{H b O_{2}}\left(\lambda_{1}\right), \varepsilon_{H b}\left(\lambda_{1}\right), \varepsilon_{H b O_{2}}\left(\lambda_{2}\right), \varepsilon_{H b}\left(\lambda_{2}\right)$ by $C_{g}$. In the clinical practice, it is reasonable to use the number of wavelengths greater than two, in order to be able to determine also the concentrations of other chromophores, significant for the optics of breast tissues. For example, if the concentrations of lipids $C_{\text {lipid }}$ and water $C_{\mathrm{H}_{2} \mathrm{O}}$ are known, then it is possible to calculate the tissue optical index using the formula [25, 28, 179, 225]

$$
T O I=C_{\mathrm{H}_{2} \mathrm{O}} C_{\mathrm{Hb}} / C_{\text {lipid }} .
$$

Getting ahead of ourselves, we note that the tissue optical index is of great importance in the breast DOT, since the visualisation of this parameter allows the enhancement of contrast between the cancer tumour and healthy breast tissue by nearly two times [25]. Not less important functional parameter is the haemoglobin concentration, $C_{t \mathrm{Hb}}=C_{\mathrm{HbO}_{2}}+C_{\mathrm{Hb}}$, which, as mentioned in Section 1, characterises the level of filling the tissue with blood. Table 2 presents some results of in vivo measurements of the functional parameters of breast tissues both in the normal condition and with pathology.

As a method of getting space-dependent data for further reconstruction and visualisation of topographic or tomographic images of the breast inner structure, the near IR spectroscopy formed the base of both the diffuse optical scanning mammography and the breast DOT. Thus, in the middle of $90 \mathrm{~s}$ the crucial breakthrough in the optical mammography occurred, when the achievements in the technology of generating and detecting optical signals combined with the progress in computer facilities and the development of algorithmic base for the reconstruction and visualisation of 2D and 3D images. This combination led to the rapid progress of the diffuse optical mammotomography.

\subsection{Diffuse optical tomography}

At resent a few international conferences and meetings held annually are devoted to the problems of DOT, among which the mammotomography topics occupy one of the priority positions. The most significant meetings are the International Congress "Photonics West" (San Francisco, USA), the International Congress on Photonics in Europe (Munich, Germany), and the International Congress "Photonics Asia" (Beijing, China). In Russia, at least two international symposia regularly take place, including the thorough discussion of DOT problems: the International Symposium "Topical Problems of Biophotonics" (Nizhny Novgorod, Russia) and the International Symposium on Optics and Biophotonics (Saratov, Russia).

Many universities and research centres are involved in the activity related to DOT studies. Among them the leaders are:

- Biomedical Optics Research Laboratory, University College London, Great Britain;

- Optical Imaging and Spectroscopy Group, University of Pennsylvania, USA;

- Photon Migration Imaging Laboratory, MGH/MIT/HMS Athinoula A. Martinos Center of Biomedical Imaging at Harvard University, USA;

- Near Infrared Imaging Group, Thayer School of Engineering at Dartmouth College, USA;

- Optical Tomography Group, SUNY Downstate Medical Center at Brooklyn, USA;

- Photon Migration Laboratory, Texas A\&M University, USA;

- Diffuse Optical Imaging of Tissue Laboratory, Science and Technology Center at Tufts University, USA;

- Functional \& Molecular Optical Imaging Laboratory, Department of Biomedical Engineering at Rensselaer Polytechnic Institute, Troy, USA. 
Table 1 Optical parameters of breast tissues in vivo.

\begin{tabular}{|c|c|c|c|c|}
\hline Tissue type & Wavelength, $\mathrm{nm}$ & $\mu_{a}, \mathrm{~cm}^{-1}$ & $\mu_{s}^{\prime}, \mathrm{cm}^{-1}$ & References \\
\hline \multicolumn{5}{|l|}{ Healthy tissue } \\
\hline \multirow{16}{*}{ bulk tissue } & 750 & $0.046 \pm 0.024$ & $8.7 \pm 2.2$ & \multirow{3}{*}{ Durduran et al. 2002 [202]* } \\
\hline & 786 & $0.041 \pm 0.025$ & $8.5 \pm 2.1$ & \\
\hline & 830 & $0.046 \pm 0.027$ & $8.3 \pm 2.0$ & \\
\hline & $674-965$ & $0.01-0.17$ & $6-11$ & Tromberg et al. 2000 [205] \\
\hline & 637 & $0.055 \pm 0.007$ & $13.4 \pm 2.6$ & \multirow{6}{*}{ Spinelli et al. 2004 [208] } \\
\hline & 656 & $0.041 \pm 0.005$ & $13.5 \pm 2.1$ & \\
\hline & 683 & $0.042 \pm 0.013$ & $12.9 \pm 2.3$ & \\
\hline & 785 & $0.037 \pm 0.013$ & $11.3 \pm 2.1$ & \\
\hline & 912 & $0.110 \pm 0.021$ & $11.4 \pm 2.6$ & \\
\hline & 980 & $0.099 \pm 0.028$ & $11.7 \pm 2.6$ & \\
\hline & 670 & $0.036 \pm 0.008$ & $10.5 \pm 1.3$ & \multirow{4}{*}{ Grosenick et al. 2005 [212] } \\
\hline & 785 & $0.039 \pm 0.011$ & $9.5 \pm 1.4$ & \\
\hline & 843 & $0.036 \pm 0.005$ & $8.4 \pm 0.4$ & \\
\hline & 884 & $0.059 \pm 0.016$ & $8.0 \pm 1.0$ & \\
\hline & 785 & $0.0503 \pm 0.0151$ & $10.53 \pm 1.20$ & \\
\hline & 808 & $0.0518 \pm 0.0153$ & $10.49 \pm 1.19$ & Mo et al. 2009 [214] \\
\hline \multirow{3}{*}{ fatty tissue } & 530 & $0.64 \pm 0.2$ & $24.1 \pm 7.8$ & \multirow{6}{*}{ Nair et al. 2002 [213] } \\
\hline & 550 & $0.65 \pm 0.1$ & $19.2 \pm 3.4$ & \\
\hline & 590 & $0.63 \pm 0.1$ & $23.2 \pm 7.2$ & \\
\hline \multirow{3}{*}{ fibrous tissue } & 530 & $0.65 \pm 0.1$ & $47.6 \pm 9.4$ & \\
\hline & 550 & $0.55 \pm 0.05$ & $46.8 \pm 7.7$ & \\
\hline & 590 & $0.56 \pm 0.8$ & $38 \pm 10.3$ & \\
\hline \multicolumn{5}{|l|}{ Benign neoplasms } \\
\hline \multirow{3}{*}{ intracanalicular } & 530 & $0.6 \pm 0.2$ & $19.3 \pm 1.4$ & \multirow{9}{*}{ Nair et al. 2002 [213] } \\
\hline & 550 & $0.7 \pm 0.02$ & $21.7 \pm 7.1$ & \\
\hline & 590 & $0.6 \pm 0.2$ & $22.3 \pm 9.9$ & \\
\hline & 530 & $0.6 \pm 0.04$ & $27.6 \pm 0.3$ & \\
\hline pericanalicular & 550 & $0.65 \pm 0.15$ & $23.4 \pm 0.2$ & \\
\hline & 590 & $1.15 \pm 0.2$ & $22.6 \pm 0.1$ & \\
\hline & 530 & $0.76 \pm 0.2$ & $20.4 \pm 3.9$ & \\
\hline mixed & 550 & $0.7 \pm 0.2$ & $19.1 \pm 3.4$ & \\
\hline & 590 & $0.7 \pm 0.2$ & $19.4 \pm 8.4$ & \\
\hline \multicolumn{5}{|c|}{ Malignant neoplasms } \\
\hline \multirow{2}{*}{ papillary cancer } & 690 & $0.084 \pm 0.014$ & $15.0 \pm 0.3$ & \multirow{2}{*}{ Fantini et al. 1998 [113] } \\
\hline & 825 & $0.085 \pm 0.017$ & $12.7 \pm 0.3$ & \\
\hline invasive ductal & 670 & 0.071 & 17.6 & Chernomordik et al. 2002 \\
\hline carcinoma & 785 & 0.042 & 16 & {$[121]$} \\
\hline \multirow{4}{*}{ carcinoma } & 670 & $0.110 \pm 0.066$ & $13.5 \pm 4.7$ & \multirow{4}{*}{ Grosenick et al. 2005 [212] } \\
\hline & 785 & $0.100 \pm 0.060$ & $11.6 \pm 3.9$ & \\
\hline & 843 & $0.118 \pm 0.096$ & $12.2 \pm 1.7$ & \\
\hline & 884 & $0.124 \pm 0.089$ & $9.1 \pm 1.9$ & \\
\hline \multirow{3}{*}{$\begin{array}{l}\text { infiltrating ductal } \\
\text { carcinoma }\end{array}$} & 530 & $0.86 \pm 0.1$ & $29.7 \pm 13$ & \multirow{3}{*}{ Nair et al. 2002 [213] } \\
\hline & 550 & $0.7 \pm 0.2$ & $28.7 \pm 13$ & \\
\hline & 590 & $0.6 \pm 0.2$ & $28 \pm 11$ & \\
\hline
\end{tabular}

*For convenience here and below in tables the references are given with the indication of the first author and publication year

Multiple large commercial companies (e.g., Hamamatsu Photonics KK, Shimadzu, Hitachi, Fuji Electric, Japan; Phillips, Netherlands; Carl Zeiss, Germany; Mallinckrodt Inc., USA) at present also carry out the research in this field. A few venture companies are especially organised for design and production of diffuse optical tomographs (e.g., Imaging Diagnostic Systems Inc.; Non-Invasive Medical Technology Inc.; ISS Inc., USA). The total world expenses for research and development in the field of DOT amount to a few hundred million dollars. Thus, e.g., the investments into only one company Imaging Diagnostic Systems Inc. exceed \$30,000,000. Actually, the leading designers of medical instrumentation permanently compete for entering the market with the tomographs of this class. The recent achievements of the Russian researchers in the field of DOT methodology allow one to expect that under favourable conditions the domestic design engineers will be able to take part in this competition as well. 
Table 2 Functional parameters of breast tissues in vivo.

\begin{tabular}{|c|c|c|c|c|}
\hline Tissue type & $\mathrm{SO}_{2}, \%$ & $C_{t H b}, \mu \mathrm{M}$ & $T O I, \mu \mathrm{M}$ & References \\
\hline \multicolumn{5}{|l|}{ Healthy tissue } \\
\hline bulk tissue & $\begin{array}{l}69 \pm 6 \\
58 \pm 9 \\
74 \pm 3 \\
74-90 \\
66.4 \pm 9.2 \\
74 \pm 7 \\
70.7 \pm 7.0 \\
67.7 \pm 9.3 \\
68 \pm 4 \\
- \\
85 \pm 7 \\
73 \pm 6 \\
68-72\end{array}$ & $\begin{array}{l}18 \pm 5 \\
22 \pm 8 \\
17 \pm 8 \\
15-49 \\
15.7 \pm 5.1 \\
17.3 \pm 6.2 \\
25.3 \pm 8.3 \\
17.5 \pm 7.5 \\
17 \pm 2 \\
36.7 \pm 8.0 \\
- \\
19 \pm 6 \\
19-22\end{array}$ & $\begin{array}{l}- \\
- \\
- \\
- \\
1.4 \pm 1.1 \\
- \\
3.3 \pm 1.9 \\
1.5 \pm 0.9 \\
1.6 \pm 0.7 \\
- \\
- \\
- \\
-\end{array}$ & $\begin{array}{l}\text { Ntziachristos et al. } 2002 \text { [198] } \\
\text { Srinivasan et al. } 2003 \text { [126] } \\
\text { Grosenick et al.2003 [129] } \\
\text { Torricelli et al. } 2003 \text { [226] } \\
\text { Spinelli et al. } 2004 \text { [208] } \\
\text { Grosenick et al. } 2005 \text { [212] } \\
\text { Tromberg et al. } 2005 \text { [225] } \\
\text { Cerussi et al. 2006 [179] } \\
\text { Wang et al. } 2010[145] \\
\text { Zhu et al. 2010 [146] } \\
\text { Yu et al. 2010 [147] } \\
\text { Fang et al. 2011 [150] } \\
\text { Enfield et al. } 2013[154]\end{array}$ \\
\hline fatty tissue & $\begin{array}{l}70.7 \pm 8.6 \\
66 \pm 12\end{array}$ & $\begin{array}{l}17.1 \pm 3.2 \\
11.3 \pm 8.3\end{array}$ & $\begin{array}{ll}- \\
-\end{array}$ & $\begin{array}{l}\text { Brooksby et al. } 2006[139] \\
\text { Srinivasan et al. } 2010[133]\end{array}$ \\
\hline fibrous tissue & $\begin{array}{l}69.7 \pm 10.4 \\
72 \pm 17\end{array}$ & $\begin{array}{l}22.4 \pm 7.3 \\
23.6 \pm 7.1 \\
\end{array}$ & $\begin{array}{l}- \\
- \\
-\end{array}$ & $\begin{array}{l}\text { Brooksby et al. } 2006 \text { [139] } \\
\text { Srinivasan et al. } 2010 \text { [133] }\end{array}$ \\
\hline \multicolumn{5}{|l|}{ Benign neoplasms } \\
\hline fibroadenoma & - & $37.6 \pm 16.0$ & - & \multirow{3}{*}{ Zhu et al. 2010[146] } \\
\hline cyst & - & $37.8 \pm 12.7$ & - & \\
\hline fat necrosis & - & $29.4 \pm 15.5$ & - & \\
\hline \multicolumn{5}{|l|}{ Malignant neoplasms } \\
\hline carcinoma & $\begin{array}{l}60 \pm 9 \\
75-77 \\
72 \pm 14 \\
68.4 \pm 8.5 \\
67.5 \pm 8.4 \\
64 \pm 5 \\
- \\
75 \pm 6 \\
62-74\end{array}$ & $\begin{array}{l}130 \pm 100 \\
33-90 \\
53 \pm 32 \\
48.5 \pm 18.2 \\
24.7 \pm 9.8 \\
28 \pm 4 \\
27.8-36.4 \\
27 \pm 14 \\
30-38\end{array}$ & $\begin{array}{l}- \\
- \\
- \\
24.9 \pm 21.5 \\
3.3 \pm 2.2 \\
6.1 \pm 1.9 \\
- \\
- \\
-\end{array}$ & $\begin{array}{l}\text { Ntziachristos et al. } 2002 \text { [198] } \\
\text { Torricelli et al. } 2003 \text { [226] } \\
\text { Grosenick et al. } 2005 \text { [212] } \\
\text { Tromberg et al. } 2005 \text { [225] } \\
\text { Cerussi et al. } 2006 \text { [179] } \\
\text { Wang et al. } 2010 \text { [145] } \\
\text { Srinivasan et al. } 2010 \text { [149] } \\
\text { Fang et al. 2011 [150] } \\
\text { Enfield et al. } 2013 \text { [154] }\end{array}$ \\
\hline $\begin{array}{l}\text { invasive ductal } \\
\text { carcinoma }\end{array}$ & $\begin{array}{l}71 \pm 4 \\
- \\
49 \pm 11 \\
- \\
\end{array}$ & $\begin{array}{l}23 \pm 5 \\
67.0 \pm 18.3 \\
51.0 \pm 13.9 \\
\end{array}$ & $\begin{array}{l}- \\
- \\
- \\
- \\
\end{array}$ & $\begin{array}{l}\text { Grosenick et al. 2003 [129] } \\
\text { Zhu et al. } 2010 \text { [146] } \\
\text { Yu et al. } 2010 \text { [147] } \\
\text { Zhu et al. } 2013 \text { [153] }\end{array}$ \\
\hline $\begin{array}{l}\text { invasive lobular } \\
\text { carcinoma }\end{array}$ & $66 \pm 7$ & $25 \pm 6$ & - & Grosenick et al. 2003 [129] \\
\hline $\begin{array}{l}\text { ductal carcinoma in } \\
\text { situ }\end{array}$ & $\begin{array}{l}- \\
58 \pm 8 \\
- \\
\end{array}$ & $\begin{array}{l}71.9 \pm 18.8 \\
- \\
72.2 \pm 24.5\end{array}$ & $\begin{array}{l}- \\
- \\
- \\
-\end{array}$ & $\begin{array}{l}\text { Zhu et al. } 2010 \text { [146] } \\
\text { Yu et al. } 2010 \text { [147] } \\
\text { Zhu et al. } 2013 \text { [153] }\end{array}$ \\
\hline
\end{tabular}

As mentioned above, in Russia the following research teams are directly involved in the DOT issues: SOI, RFNC-VNIITF, IAP RAS, MSU, and TSTU. The researchers from SOI and RFNC-VNIITF developed an original perturbation approach to solving the inverse problem of time-domain DOT. At the present stage of the study, this approach is implemented using two different methods (Sections 4.2 and 4.3). The first method, which uses for reconstruction the total bananashaped distributions of photon trajectories, according to preliminary estimations allowed the spatial resolution, record-breaking for DOT (less than $3 \mathrm{~mm}$ inside the 8$\mathrm{cm}$ object) [104, 106], while the other method, the method of photon average trajectories (PATs), demonstrated the record-breaking reconstruction time $(\sim 0.5$ s) $[5,57]$. At present, both methods are in the stage of testing in numerical phantoms. The interest of the researchers from IAP RAS is mainly concentrated on diffuse fluorescence molecular tomography (DFMT) in small laboratory animals, using the frequency-domain technique of tissue probing and signal registration [159-161]. However, they have also the experience in the field of experimental mammography [143]. To visualise the distributions of exogenous fluorophores the IAP RAS team successfully applies the original inversion methods (Section 4.2), such as the algebraic method for the minimisation of Holder norm [159] and the method of Tikhonov functional with nonnegative components [227, 228]. The researchers from MSU have assembled the prototype of a diffusion tomograph with continuous-wave radiation sources and carry out studies in phantoms. The tomograms of the phantoms visualising the probability distribution function for the detection of optical inhomogeneities are reconstructed 
using original nonlinear statistical methods ([44, 53, 54, 58, 105], Section 4.4). The researchers from TSTU mainly deal with the time-domain experiments using pulsed radiation sources. They solve the inverse DOT problem, using the so-called methods of optical inhomogeneity localisation. Recently they proposed an original localisation method based on detection of latearriving photons that form the trailing edge of the tissue response to the input pulsed signal ([92, 99, 102, 109], Section 4.4).

\section{Design problems and equipment of the diffuse optical mammotomograph}

\subsection{Design problems and operation principle of the diffuse optical mammotomograph}

The DOT technique is aimed at reconstructing and imaging the spatial distributions of optical parameters (absorption coefficient $\mu_{a}$, reduced scattering coefficient $\mu_{s}^{\prime}$, coefficient of optical diffusion $D$, anisotropy factor $g$, refractive index $n$ ) and functional parameters (tissue oxygen saturation $\mathrm{SO}_{2}$, fractional blood volume $\delta V$, concentration of haemoglobin $C_{t H b}$ and other chromophores, tissue optical index TOI) of the tissue. The parameters are extracted from the data of space-dependent measurements using the method of near IR spectroscopy. For this aim, one has to solve two main problems:

1) to design and manufacture the hardware providing generation of probing radiation, detection and primary processing of the measurement data array;

2) to develop the software capable of fast highquality reconstruction of diffusion tomograms and their presentation to a medical doctor in the form, convenient for analysing and diagnosing.

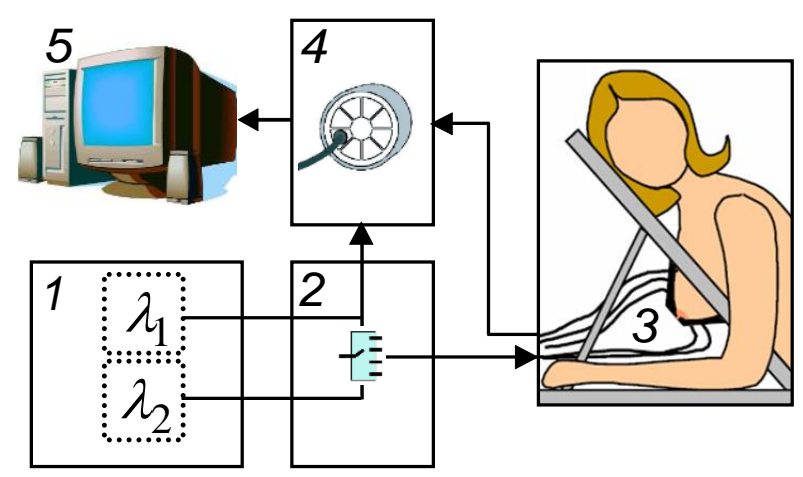

Fig. 3 Block diagram of a diffuse optical mammotomograph [5]: 1 - laser unit, 2 - channel commutator, 3 - applicator, 4 - multichannel receiver, 5 - computer.

At present, the first problem is successfully solved in the research laboratories of the world. A variety of light sources with detailed description of experimental setups, using different methods of laser radiation generation and detection of diffusely scattered light, offers a wide range of choice in technical implementations and a possibility to optimisation with respect to consumer and cost characteristics. The diffuse optical tomograph hardware includes the system of irradiating the tissue with laser light with a multichannel device for the distribution of radiation over the tissue surface, the multichannel system collecting and detecting the radiation diffusely scattered by the tissue, and the computer for the measurement data processing. To provide the possibility of functional diagnostics, it is necessary to have several sources of probing radiation with different wavelengths, or one source with the wavelength tuneable within the transparency window. A typical scheme of diffuse optical tomograph for breast examination is presented in Fig. 3 .

The setup operates as follows [5]. The breast is either placed in the ring-shaped or cup-shaped applicator (3) (Fig. 3), or softly squeezed between two parallel glass plates, like in X-ray mammography. On the surface of the applicator (plates) the source and detector optical fibres are mounted, by means of which the laser radiation enters the tissue and the diffusely scattered light is collected. The radiation from the laser unit (1) is transmitted to the applicator (plates) via the channel commutator (2) that provides sequential breast irradiation through one of the source fibres, so that the points where the radiation enters the tissue are sequentially changed. The scattered light is simultaneously collected by the receiving optical fibres and directed to the multichannel detector (4). In the detector, the signal undergoes calibrated attenuation in each channel, in order to match the level of the received signals to the dynamic range of the receiver. Then the measurement data are recorded according to the chosen technique of tissue irradiation and signal detection, and the measurement data array is input into the computer for processing and visualisation of diffusion mammotomograms.

\subsection{Techniques of tissue probing and optical signal detection}

Figure 4 schematically shows the diagrams of light intensity for three main techniques of tissue irradiation and optical signal detection used in DOT [1-6, 8, 13-18, 20, 22, 25]: the continuous-wave, the frequencydomain, and the time-domain one. In the continuouswave technique, the tissue is probed with the laser radiation of constant intensity and the transmission or diffuse reflection is measured. For quantitative interpretation of the results of measurement and for the extraction of absorption coefficient, the BouguerLambert-Beer law is used [229]. A drawback of the continuous-wave technique is the impossibility of reliable differentiation between the optical parameters $\mu_{a}$ and $\mu_{s}^{\prime}$, which, in fact, excludes further visualisation of functional parameters. Besides there are limitations of applying the method to bulk tissues, since the signal-to-noise ratio strongly depends upon the 
tissue thickness [224]. The frequency-domain technique implies the irradiation of the tissue with intensitymodulated light having the frequencies from $100 \mathrm{MHz}$ to $10 \mathrm{GHz}$, and the recording of modulation depth and phase shift of the scattered radiation at the modulation frequencies. Measuring the modulation depth and the phase shift, one can indirectly find the optical parameters $\mu_{a}$ and $\mu_{s}^{\prime}$. The time-domain technique implies the irradiation of the tissue with ultrashort pulses having the duration $10^{-9}-10^{-11} \mathrm{~s}$ and the detection of broadened pulses of scattered radiation, the so-called temporal point spread functions (TPSFs). In the time-domain near IR spectroscopy the optical parameters are assessed by comparison of the measured TPSF with the pulse shape, calculated using the diffusion approximation to the radiative transport theory (the fitting method [10]). The frequency-domain and the time-domain techniques implement time-resolved irradiation mode, the fundamental base of which is the excitation of so-called diffuse waves of photon density in the strongly scattering medium $[1-3,15,22,25]$. In comparison with the time-domain method, the frequency-domain one is simpler in implementation, since it uses the equipment already familiar in the optical communication and fibre optical dispersion studies. Thus, the required modulation frequency can be obtained using the laser diodes, and the needed dynamic range of recording is provided by using the photomultiplier tubes (PMTs) or photodiodes. To implement the time-domain technique one has to use more expensive equipment, e.g., electro-optical converter with the brightness amplifier (streak-camera) or PMT in the mode of time-correlated single photon counting (Section 3.3). However, the most important advantage of the time-domain technique is that from the TPSF one can extract different types of data, such as the integrated and logarithmic intensity, the mean time of flight for the photons, the results of Laplace and MellinLaplace transforms of the TPSF, etc. [5, 12, 224]. Just the flexibility in the interpretation of measurement results offers additional possibilities for more precise determination of optical parameters, which is particularly valuable in DOT. Thus, the researchers from SOI and RFNC-VNIITF proposed a new type of the measurement data, the time-resolved optical projections $[5,93,98,104,106,110]$. These projections are determined for a single count of the TPSF and have significant potential from the point of view of improving the DOT spatial resolution (Section 4.2).

\subsection{Instrumentation for diffuse optical tomography}

Consider in more detail the instrumentation of timedomain DOT, i.e., the instrumentation for generating the laser pulsed radiation and for the TPSF recording. The main requirement to the lasers for DOT is the maximal value of the mean power of radiation under the relatively high pulse repetition rate (more than 5-10 $\mathrm{MHz}$ ) and moderate requirements to the pulse duration (shorter than 50-100 ps). These requirements are fulfilled, e.g., in the titanium-sapphire lasers (Tsunami and Mai Tai, Spectra-Physics Lasers, USA), the picosecond semiconductor lasers (Hamamatsu Photonics KK, Japan; PicoQuant GmbH, Germany, etc.), and the fibre lasers (IMRA America Inc., USA). In Russia within the framework of the project "Development of methods of three-dimensional optical intrascopy - medical optical tomography" supported by the International Science and Technology Center (ISTC, Moscow) (grant No. 280, 2000-2002) the researchers from the Ioffe Physical and Technical Institute, Russian Academy of Sciences (PTI RAS, Saint-Petersburg) also developed high-efficiency picosecond semiconductor lasers with the characteristics optimised for DOT. Comparative characteristics of some laser sources are presented in Table 3.

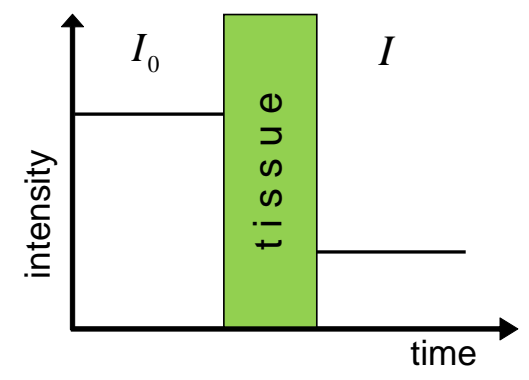

(a)

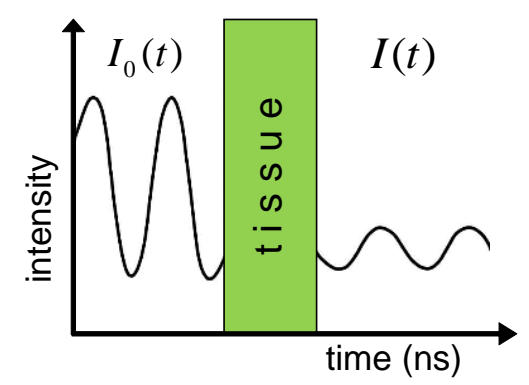

(b)

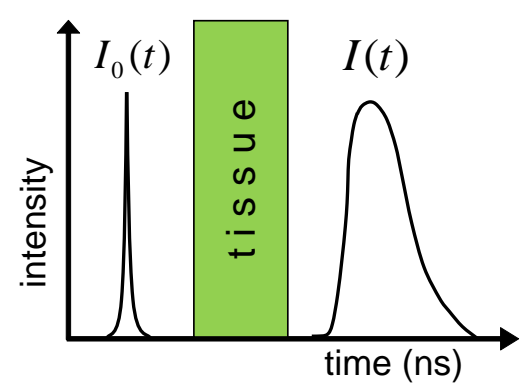

(c)

Fig. 4 The main techniques of tissue irradiation and optical signal detection: (a) - continuous-wave; (b) frequency-domain; (c) - time-domain. $I_{0}(t)$ and $I(t)$ is the light intensity before and after passing through the medium.

The titanium-sapphire lasers possess highly efficient (25-40\%) conversion of pumping radiation into the output radiation and allow much higher mean radiation power values and, therefore, peak pulse power, than the semiconductor and fibre lasers (see Table 3 ). The solidstate lasers based on crystals with metal ions possess the wavelength, tuneable within a wide spectral range 
Table 3 Comparative characteristics of lasers for pulsed DOT.

\begin{tabular}{|l|l|l|l|l|l|}
\hline \multirow{2}{*}{ Parameters } & \multirow{2}{*}{$\begin{array}{l}\mathrm{Al}_{2} \mathrm{O}_{3}: \text { Ti laser } \\
\text { Spectra-Physics* }\end{array}$} & \multicolumn{2}{|l|}{ Semiconductor laser diodes } & \multirow{2}{*}{$\begin{array}{l}\text { Fibre laser, } \\
\text { IMRA, Inc. }\end{array}$} \\
\cline { 3 - 6 } & $\begin{array}{l}\text { Hamamatsu } \\
\text { Photonics }\end{array}$ & $\begin{array}{l}\text { PicoQuant } \\
\text { GmbH }\end{array}$ & PTI RAS & 25 \\
\hline Mean radiation power, $\mathrm{mW}$ & 1000 & 0.25 & $1-4$ & 1.2 & 2 \\
\hline Pulse duration, ps & 0.1 & 100 & $180-400$ & 40 & 40 \\
\hline Pulse repetition rate, $\mathrm{MHz}$ & 80 & 5 & 40 & 10 & 2 \\
\hline
\end{tabular}

*The data are presented for the $\mathrm{Al}_{2} \mathrm{O}_{3}$ :Ti laser Mai Tai

(750-850 nm for $\mathrm{Al}_{2} \mathrm{O}_{3}$ : Ti laser Mai Tai [230]). However, such lasers are sensitive to the conditions of exploitation and have rather large overall dimensions. Therefore in time-domain DOT they more frequently use portable semiconductor laser diodes $[115,121,129$ 132, 231]. The required pulse duration of such laser is achieved using the implantation of ultrafast saturable absorbers, and the increase of the pulse power in the Qmodulation mode is provided by the increase of the total number of nonequilibrium carriers in the laser cavity and the depth of modulation of the concentration of these carriers. Undisputable prospects for using in DOT are inherent also in the fibre lasers thanks to their compactness, combining the laser and waveguide functions, and the possibility of their use as a base for designing multichannel laser systems. The active medium of fibre lasers is created either due to using the nonlinear properties of the fibre material, or by using the appropriate laser materials for the core fabrication. Using different active fibres one can conveniently vary the wavelength within a wide range. Multichannel systems are implemented using the bundles of multiple active fibres. At present the fibre lasers IMRA America Inc. are, e.g., used in the well-known setup Multichannel Opto-electronic Near-infrared System for Timeresolved Image Reconstruction (MONSTIR, University College London, Great Britain) [137, 140, 154, 224, 230].

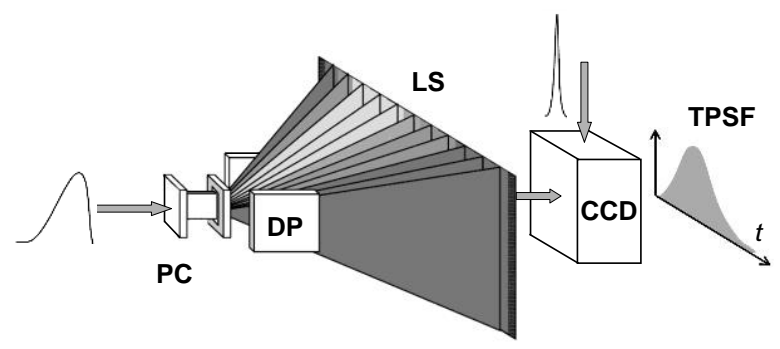

Fig. 5 Schematic of a single-channel streak camera for DOT [5]

To detect the scattered light in time-domain DOT, as well as in time-domain near IR spectroscopy, they use streak-cameras or PMTs for time-correlated single photon counting. The detailed description of a singlechannel streak-camera intended for DOT is presented, e.g., in Refs. [5, 232]. Figure 5 schematically illustrates the principle of its operation. The photons of the broadened response to the picosecond pulse from the input pulse train, generated by the laser, bombard the photocathode (PC) and are converted into electrons. Two deflection plates (DP), to which the sweep variable voltage is applied, accelerate the electrons that arrive at the luminescent screen (LS). The CCD matrix implements data readout. The inclination of electrons depends upon the sweep voltage. The electrons that reach a definite position along the screen horizontal always spend the same time with respect to the input pulse. Thus, if the frequency of the sweep voltage coincides with the repetition rate of the input pulses, then the CCD camera will record the profile, coinciding with the TPSF. The streak-camera is a large-size device with a limited field of view, nonlinear temporal characteristic and relatively narrow dynamic range, not adapted for use in multichannel detection systems. Therefore, in time-domain DOT the digital registration is preferred with the use of multi-cathode micro-channel PMTs operating in the mode of time-correlated single photon counting [224, 231, 233-235].

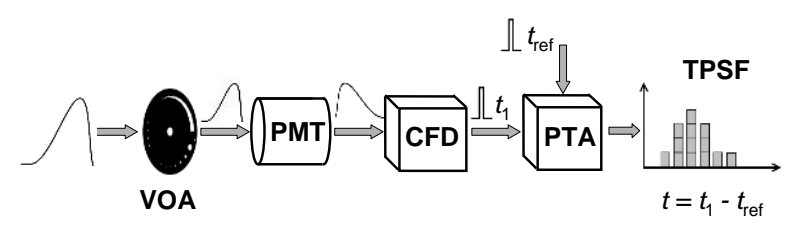

Fig. 6 Schematic of the recording channel with PMT in the mode of time-correlated single photon counting [5].

Figure 6 schematically illustrates the operation principle of a single channel of such recorder. Via the receiving fibre, the broadened response to the input pulse arrives at the variable optical attenuator (VOA), where its amplitude is reduced to the required magnitude. The PMT detects only one photon and forms the electron pulse corresponding to it. This pulse is transformed into the digital one by means of the constant fraction discriminator (CFD). The CFD also executes the exact load-time binding of the pulse. The picosecond time analyser (PTA) measures and stores its time delay with respect to the reference pulse, formed in the reference registration channel. After processing a sequence of broadened responses, the histogram of the TPSF is recorded. A certain disadvantage of this approach is that the recorded TPSF is amplitudenormalised. Therefore, if in the time-domain 
experiments one has to differentiate spatio-temporal signals by intensity (see, e.g., [92, 99, 102, 109]), the registration should be executed with a streak-camera.

At present the setups for experimental diffuse optical mammotomography most known in the world are:

- MONSTIR [137, 140, 154, 224, 230];

- Dynamic Near-infrared Optical Tomographic Imaging System (DYNOT, SUNY Downstate Medical Center at Brooklyn, USA) [236];

- Near Infrared Tomography Breast Imaging System (Thayer School of Engineering at Dartmouth College, USA) [116, 118, 120, 122, 125, 126, 133, 134, 139];

- Diffuse Optical Tomography System for Breast Cancer Imaging (University of Pennsylvania, USA) [123, 138, 144];

- Mammograph for Optical Tomography (MAMMOT, Politecnico di Milano, Italy) [131, 132, 208, 226];

- Scanning Laser Pulse Mammograph (Physikalische-Technische Bundesanstalt, Germany) [111, 113, 115, 121, 129, 130];

- System for Near-infrared Spectral Imaging of the Breast (Technology Center at Tufts University, USA) [101, 147, 155].

These setups and many other ones use different techniques of tissue irradiation and optical signal registration, different combinations of sources and receivers, different constructions of gantry for breast positioning. Thus, e.g., the European groups from Great Britain, Italy and Germany prefer the pulsed irradiation, while the Americans tend to frequency-domain technique. The researchers from the Pennsylvania University use the original combination of continuouswave and frequency-domain techniques of probing. The number of sources in the setups with fixed geometry varies from 24 to 128 [236]. The corresponding figures for the number of receivers have even greater spread from 8 [117] to 993 [123, 128]. In the scanning systems developed in Italy and Germany at each operating wavelength one source and one receiver are used, the number of their positions varying within $1000-2000$. As to the gantry constructions, the breast, as mentioned above, can be positioned inside the applicator (Fig. 3), or softly compressed between two parallel glass plates. In the first case, the geometry is cylindrical, conical, or spherical, and in the second case, the geometry has the symmetry of a planar layer. Note, that using the applicator they fill the free space between the breast and the applicator with contact gel with optical propertiessimilar to those of the tissue in order to match the refractive indices. The base of such gel is usually water, gelatine or hydrogel, and the role of scattering and absorbing agents is played by the fat-containing emulsions and different dyes (ink, Indian ink, etc.), respectively [78]. In the case of parallel plates, no gel is needed, since due to the breast compression the air gap between the tissue and the plates is absent.

The experimental setup for frequency-domain DFMT in laboratory animals developed by the IAP RAS team is described, e.g., in Ref. [159]. As a source of radiation modulated with the frequency $1 \mathrm{kHz}$, the $\mathrm{Nd}$ :
YAG laser is used that generates the second harmonic at the wavelength $532 \mathrm{~nm}$. To detect the signal of the fluorescence response they use the Hamamatsu PMT (PMT Hamamatsu H7422-20). The space-dependent data are recorded in the transmission geometry of a planar layer, the fibre-optical output of the source and the fibre-optical input of the receiver synchronously moving with the specified scanning step. In the setup adapted to mammography studies [143] the fibre lasers with the wavelengths $684 \mathrm{~nm}, 794 \mathrm{~nm}$, and $850 \mathrm{~nm}$ are used, and the modulation frequency is equal to 140 $\mathrm{MHz}$. The use of the same principle of synchronous scanning makes the data recording time undesirably long, 10-30 minutes, depending on the breast size.

The experimental prototype of a diffusion tomograph developed in MSU is equipped with the continuous-wave sources, i.e., the semiconductor laser diodes with fibre-optical radiation output. In the differential version of the tomograph [105], two such diodes are used operating at the wavelengths $790 \mathrm{~nm}$ and $815 \mathrm{~nm}$. The setup is intended for "transillumination" of cylindrical scattering objects in the form of vessels with fat emulsion, along the perimeter of which 32 input and 32 output optical fibre waveguides are glued in. Two Hamamatsu PMTs (PMT Hamamatsu H6240-02) operating in the mode of timecorrelated single photon counting are used as detectors. The complete data set is obtained by sequential switching of one input channel and two output channels using the commutator.

The TSTU team constructed a setup for experimenting with cylindrical phantoms [92, 99, 102, 109]. As a source of radiation, they use the mode-locked titanium-sapphire laser MIRA 900-B. The laser pulse duration is $100 \mathrm{fs}$ and the wavelength is $730 \mathrm{~nm}$. The nonnormalised TPSFs are recorded by means of the Hamamatsu streak-camera (Streak Camera Hamamatsu C4334) with the time resolution $10 \mathrm{ps}$. The studied scattering phantom is a cylinder made of epoxy resin with the admixture of $\mathrm{TiO}_{2}$. Cylindrical inserts of the same material stained with the Indian ink simulate the absorbing inhomogeneities. The method used to solve the problem of the inhomogeneity localisation (Section 4.4) implies the data pickup for a single position of the source and 10 positions of the detector, the optical inputs of which are arranged with equal angular step along the phantom perimeter.

\section{Methods of diffusion tomogram reconstruction}

In contrast to the problem of hardware design for diffusion tomography, the problem of developing software for DOT image reconstruction still has no completed commercial solution. In spite of the rapid progress of computer technologies, there are still no precise and fast algorithms for high-quality reconstruction of diffusion tomograms in the time scale, acceptable for clinical studies. This fact is the main reason why diffuse optical tomographs are still absent at the world market of instrumentation for medical imaging. Indeed, the problem of image reconstruction in 
DOT is much more complex than, e.g., in X-ray or ultrasonic tomography. The reason is that due to multiple scattering of light the dependence of the measured data upon the reconstructed optical parameters is nonlinear in principle. At present a variety of methods for solving the inverse DOT problem are developed. The conventional classification of methods is presented in Fig. 7.

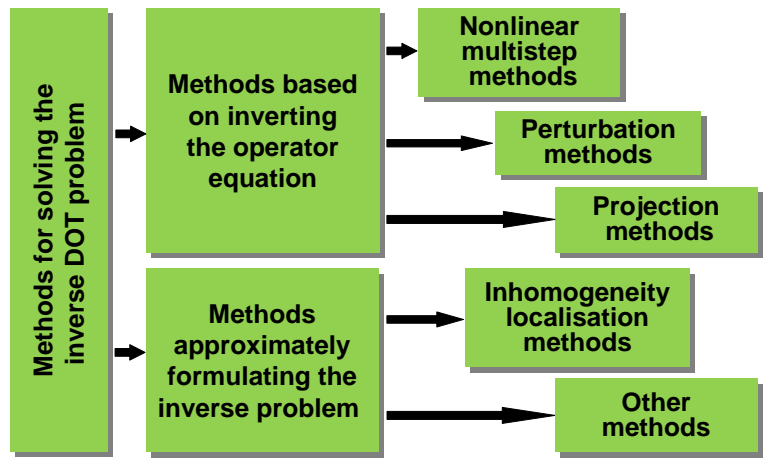

Fig. 7 Classification of methods for solving the inverse DOT problem.

\subsection{Methods based on inverting the operator equation}

The most general mathematical formulation of the inverse DOT problem is the problem of inverting the nonlinear operator equation

$$
g\left(\mathbf{r}_{s}, \mathbf{r}_{d}\right)=\Phi[f(\mathbf{r})]
$$

where $g\left(\mathbf{r}_{s}, \mathbf{r}_{d}\right)$ is the result of measuring the optical signal for the positions $\mathbf{r}_{s}$ of the source and $\mathbf{r}_{d}$ of the detector at the boundary of the studied scattering object, $f(\mathbf{r})$ is the desired function of optical parameter distribution (object function). The particular form of the functions $g\left(\mathbf{r}_{s}, \mathbf{r}_{d}\right)$ and $f(\mathbf{r})$ depends on the technique and geometry of tissue irradiation and data registration, as well as on the chosen reconstruction method.

In contrast to the Radon equation underlying the projection tomography, the operator equation (5) has no general analytical solution. Therefore, it is commonly inverted using the discrete model, according to which the $3 \mathrm{D}$ reconstruction domain is divided into a set of volume elements. Each volume element $(i, j, k)$ is characterised by its discrete value of the object function $f_{i, j, k}$. The reconstruction problem is reduced to the search for the best estimate of the vector $\mathbf{f}=\operatorname{vec}\left\{f_{i, j, k}\right\}$, satisfying the operator equation

$$
\mathbf{g}=\Phi[\mathbf{f}]
$$

where $\mathbf{g}$ is the vector of measurement data, and the operation "vec" denotes the rearrangement of a 3D array into a vector. The traditional approach to the estimation of $\mathbf{f}$ consists in the linearization of Eq. (6) by expanding it into the Taylor series $[3,5,9,10,12,17]$
$\mathbf{g}=\Phi\left[\mathbf{f}_{0}\right]+\Phi^{\prime}\left[\mathbf{f}_{0}\right]\left(\mathbf{f}-\mathbf{f}_{0}\right)+\frac{1}{2}\left(\mathbf{f}-\mathbf{f}_{0}\right)^{T} \Phi^{\prime \prime}\left[\mathbf{f}_{0}\right]\left(\mathbf{f}-\mathbf{f}_{0}\right)+\ldots$,

where $\mathbf{f}_{0}$ is the initial approximation for $\mathbf{f}$, a $\Phi^{\prime}\left[\mathbf{f}_{0}\right]=\mathbf{J}$ and $\Phi^{\prime \prime}\left[\mathbf{f}_{0}\right]=\mathbf{H}$ are the Jacobian and Hessian matrices, the analogues of Fréchet derivatives of the first and the second order in the continuous case. If, e.g., one neglects the third and the higher terms of the expansion, the system of linear algebraic equations (SLAE) follows

$$
\Delta \mathbf{g}=\mathbf{J} \Delta \mathbf{f},
$$

where $\Delta \mathbf{g}=\mathbf{g}-\Phi\left[\mathbf{f}_{0}\right], \Delta \mathbf{f}=\mathbf{f}-\mathbf{f}_{0}$.

The reconstruction methods based on the singe inversion of the system (8) and using only single-step linearization of the operator equation (6) are known as linear or perturbation methods $[3,5,9,12,14,15,17$, 19-22, 32, 34-36, 47, 49, 52, 56, 57, 59, 64, 66, 79, 80, 84, 93, 98, 104, 106, 110, 117, 159, 224, 227, 228]. Before the procedure of Eq. (8) inversion, the vector $\Phi\left[\mathbf{f}_{0}\right]$ and the matrix $\mathbf{J}$ should be determined basing on a certain exact model of radiation, propagating through the matter, e.g., the radiative transport theory $[1-6,10$, $12,14,15,21,41,59,62,82,95]$, its diffusion approximation $[1-17,18-25,29-40,43,45-49,52,56$, 57, 59-61, 63-73, 75-77, 79-81, 83-91, 93, 94, 96-98, $100,103,104,106-108,110,114,116-120,122-128$, $133-145,159-161,224,227]$, or the Monte Carlo method [10, 51]. These calculations do not require considerable time expenditures only in particular cases of homogeneous scattering objects with simple geometries, when the known analytical solutions of the radiative transport equation can be applied $[5,6,10,22$, $32,43,46,52,69,80,83,93,98,104,106,110,115$, $224,237]$. In the general case one has to address the labour-consuming numerical methods, such as the finite element method $[3,5-7,12,19,21,29,37,45,59,76$, $91,125,224]$, finite difference method $[12,19,21,40$, $41,55,62,123]$, and boundary element method [19, 21, $141,149]$.

The inversion of the system (8) itself is nontrivial, since the system, overdetermined or underdetermined in the case of strong data deficiency, is inconsistent and the matrix $\mathbf{J}$ is ill conditioned. Therefore, there is no solution of Eq. (8) in the common sense, $\Delta \mathbf{f}=\mathbf{J}^{-1} \Delta \mathbf{g}$. That is why in order to invert Eq. (8) they use the methods with regularisation. Tikhonov regularisation is most frequently used, which implies the minimisation of the functional $[5,13,21,61,77,90,97,124,139,238]$

$$
\psi(\Delta \mathbf{f})=\|\Delta \mathbf{g}-\mathbf{J} \Delta \mathbf{f}\|_{2}^{2}+\lambda\|\Delta \mathbf{f}\|_{2}^{2}
$$

where $\|\cdot\|_{2}$ denotes the $L_{2}$ (Euclidean) norm, and $\lambda$ is the regularisation parameter. When using the direct Tikhonov inversion, the solution of Eq. (8) is written in the form (see, e.g., [77]) 


$$
\Delta \mathbf{f}=\left[\mathbf{J}^{T} \mathbf{J}+\lambda \mathbf{I}\right]^{-1} \mathbf{J}^{T} \Delta \mathbf{g},
$$

in the case of overdetermined Jacobian matrix, and in the form

$$
\Delta \mathbf{f}=\mathbf{J}^{T}\left[\mathbf{J} \mathbf{J}^{T}+\lambda \mathbf{I}\right]^{-1} \Delta \mathbf{g},
$$

in the case of underdetermined one. In Eqs. (10) and (11) I is the identity matrix. Practically to solve the problem of minimising the functional (9) they more frequently use the iteration algorithms of least squares [5, 57, 239, 240], conjugate gradients [5, 12, 51, 241], steepest descent $[5,21,242]$, algebraic techniques [5, $10,12,21,57,80,83,84,93,98,104,106,110,117$, 159, 227, 228, 243, 244], Newton-type algorithms [12, 21, 23, 77, 224, 245], etc.

As a rule, the linear reconstruction methods are efficient only when the small perturbation theory is valid [246, 247], i.e., when the optical inhomogeneities are small-scale and possess small amplitude. Otherwise, one step of linearization is not enough and the system (8) has to be inversed multiply, solving the forward problem at each step and repeatedly calculating the matrix J . Such multistep procedure implying the optimisation of the functional of the general form

$$
\psi(\mathbf{f})=\|\mathbf{g}-\Phi[\mathbf{f}]\|_{2}^{2}+\lambda \Omega[\mathbf{f}]
$$

to get the regularised solution $\mathbf{f}^{(s+1)}=\mathbf{f}^{(s)}+\Delta \mathbf{f}^{(s)}$ at a certain $(s+1)$-th step of approximation, underlies the nonlinear reconstruction methods $[3,5,7,9,12,17,19$, 21-23, 29, 31, 33, 37, 40, 41, 45, 55, 62, 70, 77, 89, 91, $95,103,123-125,224]$. In Eq. (12) $\Omega[\mathbf{f}]$ is the stabilising functional. If the Tikhonov regularisation is used, then, obviously $\Omega[\mathbf{f}]=\|\mathbf{f}\|_{2}^{2}$. In recent years multiple publications appeared where the efficiency of minimisation of the Holder norm $L_{p}$ at $0 \leq p<2$ (see, e.g., $[159,248-254])$, or the total variation norm ( $T V$ norm) $[3,33,110]$ is justified. In these cases the following relations are valid for the stabilising functional

$$
\Omega[\mathbf{f}]=\|\mathbf{f}\|_{p}^{p},
$$

where $\|\mathbf{f}\|_{p}=\left(\sum_{i, j, k}\left|f_{i, j, k}\right|^{p}\right)^{1 / p}$ is the Holder norm, or

$$
\Omega[\mathbf{f}]=\|\mathbf{f}\|_{T V},
$$

where

$\|\mathbf{f}\|_{T V}=$

$=\sum_{i, j, k} \sqrt{\left(f_{i, j, k}-f_{i-1, j, k}\right)^{2}+\left(f_{i, j, k}-f_{i, j-1, k}\right)^{2}+\left(f_{i, j, k}-f_{i, j, k-1}\right)^{2}}$

is the total variation norm.
Sometimes one can achieve higher accuracy of reconstruction, if a certain orthonormal transform $\hat{\mathbf{f}}=\mathrm{T}[\mathbf{f}]$ is found, in the space of which the reconstructed signal (the image f ) satisfies the principle of transform sparsity. The sparsity criterion is the condition $\|\hat{\mathbf{f}}\|_{p} \leq R$, where $R$ is a certain positive number [255]. In this case the functional (12) is written and minimised already for the signal in the space of the transform (for the image $\hat{\mathbf{f}}$ ). Such approach, called compressive sensing or compressive sampling [255257], is successfully applied in DOT [248, 249, 252, 254], but is even more used in DFMT [258-272]. As seen from the presented references, the publication boom falls on the recent 3-4 years. The explanation is that due to small dimensions of the laboratory animals for DFMT a real possibility exists to approach the millimetre and submillimetre spatial resolution, which is of extreme importance for experimental oncology.

To solve the problem of minimising (12) a variety of multistep reconstruction methods are developed with the iterative correction of the Jacobian (and sometimes Hessian) matrix. These methods are mainly based on the Bayesian algorithms $[42,50,68,73,76]$, the Newtontype algorithms $[3,5,7,12,21,29,33,37,59-62,70$, $77,91,116,119,123-125,224]$, and the gradient-type algorithms [12, 21, 40, 41, 45, 55, 59]. In the case of minimising the norm $L_{1}$ (Holder norm for $p=1$ or Manhattan norm) the linear programming algorithms are exploited, too [273]. As an example, Figure 8 presents the key diagram of a multistep algorithm, based on multiple linearization of the reconstruction problem.

Having reconstructed the spatial distribution of the absorption coefficient at different wavelengths and using, e.g., Eqs. (2) - (4), one can visualise the distributions of different functional parameters, playing important role in the diagnostics of oncologic diseases.

At present in the world, a few software complexes exist in which the multistep reconstruction algorithms are successfully implemented for $2 \mathrm{D}$ and $3 \mathrm{D}$ cases. The most well-known are the package TOAST (Temporal Optical Absorption and Scattering Tomography, University College London, Great Britain) [29, 45, 224, 274] and NIRFAST (Near Infrared Fluorescence and Spectral Tomography, Thayer School of Engineering at Dartmouth College, USA) [275, 276]. Note, that the field of application of these packages is not restricted to DOT as such, but also includes the DFMT and the diffuse optical correlation tomography.

Due to multiple light scattering, the spatial resolution of diffusion tomograms is essentially worse than the resolution provided by other methods of medical imaging, such as X-ray CT, MRT, and ultrasonic reflectivity tomography. According to Refs. $[5,35,43,57,75,83,84,104]$ it can be characterised by the value of root-mean-square (RMS) deviation of photons, migrating from the point of emission to the point of detection, from their average statistical trajectory. This value mainly depends upon the object dimensions and the depth of the visualised structure location. Thus, the resolution in DOT is a spatially varying quantity. Multistep algorithms implementing 
the nonlinear reconstruction methods, are the most precise ones and allow the resolution about 4-6 $\mathrm{mm}$ in the depth of the scattering object with the dimension 10$12 \mathrm{~cm}$, and 1-3 mm near the boundary of the object [77]. Although for the reason mentioned in the Introduction the oncologists are not fully satisfied with the above value of in-depth resolution, they partially are ready to agree with it, since the contrast of tumourvisualising images appears quite satisfactory for setting a correct diagnosis. This is explained by the fact that the quantitative values of optical and functional parameters can strongly differ in healthy and cancer tissues (see, in particular, Tables 1 and 2). Thus, e.g., according to the analysis of Refs. [113, 121, 202, 205, 208, 212-214] the absorption coefficient in the therapeutic transparency window varies within the limits from 0.01 [205] to 0.65 [213] $\mathrm{cm}^{-1}$ in healthy breast tissue and from 0.042 [121] to $0.86[213] \mathrm{cm}^{-1}$ in carcinoma. It is interesting to note that the absorption coefficient of benign neoplasms varies from $0.6 \pm 0.2$ to $1.15 \pm 0.2 \mathrm{~cm}^{-1}$ [213]. According to Ref. [212], the most probable ratio of absorption coefficients of cancer tumour and healthy breast tissue lies within the range $1.5-2$, and the ratio of reduced scattering coefficients in the range $1-1.25$. It is also necessary to allow for the difference in refractive index between the normal tissues and malignant neoplasms, which should be taken into account in the tomographic reconstruction algorithms. Thus, e.g., the refractive index of normal breast tissue at the wavelength $800 \mathrm{~nm}$ amounts to 1.403 , while in the case of malignant neoplasm it is 1.431 [10]. As to the functional parameters, from Table 2 it is seen that the ranges of characteristic values of haemoglobin oxygen saturation essentially overlap in healthy breast tissue and malignant tissue, namely, $49-92 \%$ and $38-81 \%$. However, the ratio of haemoglobin concentrations of a cancer tumour and healthy tissue lies within $1.4-1.9$ $[129,145,146,150,154,179,225,226]$, and the ratio of tissue optical indices within $2.2-7.5$ [145, 179, 225]. These facts confirm the possibility of getting good contrast in visualising the corresponding parameters and, therefore, successful recognition of malignant neoplasms against the background of the healthy breast tissues.

The analysis of reconstruction time using multistep algorithms is presented in Refs. [41, 70, 88, 91, 103, 107, 125, 140, 224, 275]. According to Refs. [125, 224, 275 ], for getting a regularized solution it is enough to perform nearly 10 iterations. If a computer (or workstation) with central processing units is applied, one iteration takes a few minutes to reconstruct a 2D image, and a few tens of minutes for a 3D one. Some data on the computation time with the indication of number of iterations, number of finite elements of the used mesh, and the computer parameters are summarised in Table 4. Apparently, the values shown in Table 4 cannot satisfy current requirements of medical diagnostics. However, one can trace positive dynamics of reducing the reconstruction time in the last years. The major success is, apparently, due to the use of graphics processing units (GPUs) for parallel computations [88, $91,103,107,275]$. We can expect that the next step in the development of processor technique will allow the ultimate solution of the computing rate problem in DOT.

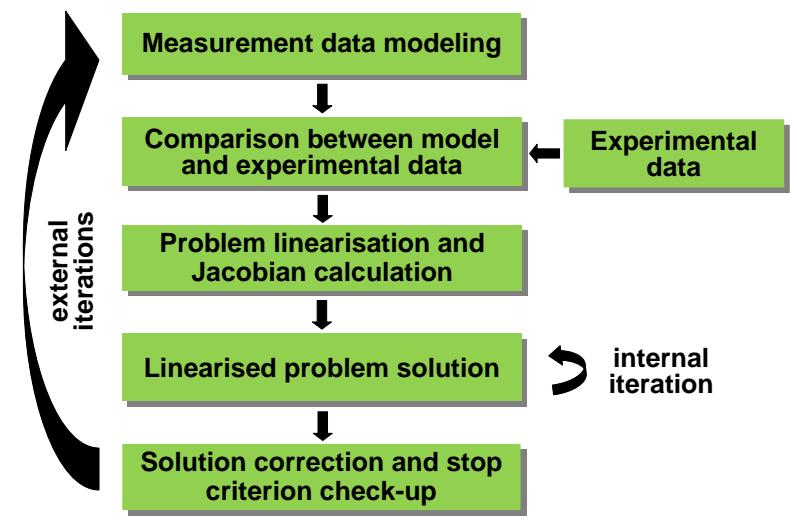

Fig. 8 Block diagram of the multistep reconstruction algorithm.

In spite of this undoubtful prospect, today the researchers continue to seek a compromise between the reconstruction accuracy of multistep algorithms and the high speed of algorithms implementing approximate methods of solving the inverse DOT problem. In the review of approximate DOT methods presented below from the point of view of accuracy and speed of reconstruction, it is reasonable to select three large groups: the perturbation semianalytic methods, the methods of projection tomography (or projection methods), based on the fast inversion of Radon equation, and the methods approximately formulating the inverse DOT problem.

\subsection{Perturbation semianalytic methods}

The most obvious way to simplify and accelerate the reconstruction procedure is to use the perturbation methods, in the first approximation neglecting the nonlinearity of the problem. However, as mentioned above, the implementation of a single step of linearization (i.e., a single step of the multistep reconstruction) using the numerical methods already requires significant time (see Table 4). Hence, they widely use various versions of the perturbation method based on the known analytic solutions of the optical diffusion and radiative transport equations to calculate the matrix of the SLAE (i.e., the Jacobian matrix), describing the linearized reconstruction problem. Such methods, S.R. Arridge called the semianalytic ones [12], have been studied by many authors $[5,12,22,31,34$, $36,41,47,52,56,57,59,64,66,69,80,82-84,93,98$, $104,106,110,123,127,227]$. All these methods reduce the inverse DOT problem to the solution of the linear Fredholm integral equation of the first kind

$$
g\left(\mathbf{r}_{s}, \mathbf{r}_{d}\right)=\int_{V} W\left(\mathbf{r}_{s}, \mathbf{r}_{d}, \mathbf{r}\right) f(\mathbf{r}) d^{3} r,
$$

where $V$ is the volume of the scattering object, $W\left(\mathbf{r}_{s}, \mathbf{r}_{d}, \mathbf{r}\right)$ is the weight function, accounting for the 
Table 4 Reconstruction time for multistep algorithms.

\begin{tabular}{|c|c|c|c|c|c|}
\hline $\begin{array}{l}\text { Number of } \\
\text { iterations }\end{array}$ & $2 \mathrm{D}$ or $3 \mathrm{D}$ & $\begin{array}{l}\text { Number of mesh } \\
\text { finite elements }\end{array}$ & $\begin{array}{l}\text { Reconstruction } \\
\text { time }\end{array}$ & Computer & References \\
\hline 20 & $2 \mathrm{D}$ & 6400 & 12 hours & $\begin{array}{l}350 \mathrm{MHz} \\
\text { PentiumII LINUX } \\
\text { Workstation }\end{array}$ & Klose et al. 1999 [41] \\
\hline 10 & $2 \mathrm{D}$ & 6615 & 36 minutes & $\begin{array}{l}400 \mathrm{MHz} \\
\text { Sun Ultra-10 } \\
\text { Workstation }\end{array}$ & \multirow{2}{*}{$\begin{array}{l}\text { Hillman et al. } 2002 \\
\text { [224] }\end{array}$} \\
\hline 6 & $3 \mathrm{D}$ & 36857 & 38 hours & $\begin{array}{l}450 \mathrm{MHz} \\
\text { Pentium PC } \\
\text { 1GB RAM }\end{array}$ & \\
\hline \multirow{2}{*}{10} & \multirow{2}{*}{$3 \mathrm{D}$} & $\begin{array}{l}107918 * \\
4151 * *\end{array}$ & 3.3 hours & \multirow{2}{*}{$\begin{array}{l}1,7 \mathrm{GHz} \\
\text { Pentium PC } \\
\text { 2GB RAM }\end{array}$} & \multirow{2}{*}{$\begin{array}{l}\text { Dehghani et al. } 2003 \\
\text { [125] }\end{array}$} \\
\hline & & $\begin{array}{l}38725^{*} \\
16556^{* *}\end{array}$ & 1.6 hours & & \\
\hline 4 & $3 \mathrm{D}$ & 336030 & 1 hour & $\begin{array}{l}3,2 \mathrm{GHz} \\
\text { Pentium PC } \\
\text { 2GB RAM }\end{array}$ & $\begin{array}{l}\text { Schweiger et al. } 2005 \\
\text { [70] }\end{array}$ \\
\hline 10 & $3 \mathrm{D}$ & 46415 & 8 hours & $\begin{array}{l}2,2 \mathrm{GHz} \\
\text { Xeon PC } \\
\text { 800MB RAM }\end{array}$ & $\begin{array}{l}\text { Enfield et al. } 2007 \\
\text { [140] }\end{array}$ \\
\hline 9 & $2 \mathrm{D}$ & $\begin{array}{l}9570 * \\
3418 * *\end{array}$ & 10 minutes & \multirow{2}{*}{$\begin{array}{l}\text { 3,7GHz } \\
\text { LINUX Redhat } \\
\text { Workstation } \\
\text { 4GB RAM }\end{array}$} & \multirow{2}{*}{$\begin{array}{l}\text { Dehghani et al. } 2009 \\
\text { [275] }\end{array}$} \\
\hline 11 & $3 \mathrm{D}$ & $\begin{array}{l}215208^{*} \\
75215^{* *}\end{array}$ & 5 hours & & \\
\hline 1 & $2 \mathrm{D}$ & $\sim 20000$ & 2 minutes & \multirow{2}{*}{$\begin{array}{l}1,33 \mathrm{GHz} \\
\text { GPU NVIDIA } \\
\text { Tesla C1060 } \\
\text { 4GB RAM }\end{array}$} & \multirow[b]{2}{*}{$\begin{array}{l}\text { Prakash et al. } 2010 \\
\text { [88] }\end{array}$} \\
\hline 1 & $3 \mathrm{D}$ & $\sim 36000$ & 7 minutes & & \\
\hline 1 & $3 \mathrm{D}$ & 444278 & $18-25$ minutes & $\begin{array}{l}1,47 \mathrm{GHz} \\
\text { GPU NVIDIA } \\
\text { GTX } 285 \\
\text { 1GB RAM } \\
\end{array}$ & $\begin{array}{l}\text { Schweiger et al. } 2011 \\
\text { [91] }\end{array}$ \\
\hline 1 & $3 \mathrm{D}$ & 66314 & $20-30$ seconds & $\begin{array}{l}0,7 \mathrm{GHz} \\
\text { GPU NVIDIA } \\
\text { Tesla K20C } \\
\text { 5GB RAM }\end{array}$ & $\begin{array}{l}\text { Saikia et al. } 2014 \\
\text { [103] }\end{array}$ \\
\hline 6 & $3 \mathrm{D}$ & $\begin{array}{l}9972 \\
\sim 14000 \\
\sim 20000\end{array}$ & $\begin{array}{l}2.8 \text { seconds } \\
5.0 \text { seconds } \\
7.6 \text { seconds }\end{array}$ & $\begin{array}{l}3,4 \mathrm{GHz} \\
\text { GPU NVIDIA } \\
\text { GTX 590 } \\
\text { 16GB RAM }\end{array}$ & $\begin{array}{l}\text { Zhang et al. } 2015 \\
\text { [107] }\end{array}$ \\
\hline
\end{tabular}

* solution of forward problem, ** image reconstruction

contribution of each point of the object to the formation of the signal between the points $\mathbf{r}_{s}$ and $\mathbf{r}_{d}$. In fact, the semianalytic methods, using the Born [246] or Rytov [247] perturbation model, determine a certain analytic (or semianalytic) representation of the function $W\left(\mathbf{r}_{s}, \mathbf{r}_{d}, \mathbf{r}\right)$. The matrix of SLAE is obtained as a result of the discretisation and joining the weight functions, calculated for different source-detector links. Thus, V.A. Markel, who studied the frequency-domain technique of data recording, proposed the following simple and elegant formulae [52]:

$$
W_{\mu_{a}}\left(\mathbf{r}_{s}, \mathbf{r}_{d}, \mathbf{r}\right)=G\left(\mathbf{r}_{s}, \mathbf{r}\right) G\left(\mathbf{r}, \mathbf{r}_{d}\right)
$$

for visualising the distribution of absorbing inhomogeneities and

$$
W_{D}\left(\mathbf{r}_{s}, \mathbf{r}_{d}, \mathbf{r}\right)=\nabla G\left(\mathbf{r}_{s}, \mathbf{r}\right) \nabla G\left(\mathbf{r}, \mathbf{r}_{d}\right)
$$

for the scattering ones. In Eqs. (16) and (17) $G\left(\mathbf{r}^{\prime}, \mathbf{r}\right)$ is the Green function of the Helmholtz equation for the amplitude of the frequency dependent component of the photon density.

From the point of view of a compromise between the accuracy and speed of reconstruction, the semianalytic methods occupy the intermediate position between the nonlinear methods and the methods of projection tomography. The neglect of nonlinearity and the inevitable limitations in choosing the geometry and the initial approximation, as a rule, negatively affect the reconstruction accuracy. On the other hand, the inversion (even single) of the large-dimension matrix with nonzero elements requires iteration algebraic techniques with regularization, which are not the fastest 
ones $[5,10,12,21,57,80,83,84,93,98,104,106,110$, $117,159,227,228,243,244]$. However, to the opinion of the authors, the semianalytic methods of time-domain DOT have a perspective from the point of view of both improving the reconstruction accuracy and minimisation of processing time. To achieve higher spatial resolution one has to use for reconstruction the new types of data extracted from the TPSF. The reduction of the processing time is possible by paralleling the iterative reconstruction procedure.

In late 90-s - early 2000-s V.V. Lyubimov from SOI proposed an original perturbation model for timedomain DOT [35, 36, 43, 46, 47]. Then this model, below referred to as Lyubimov model, was developed by joint efforts of the researchers from SOI and RFNCVNIITF [5, 57, 59, 66, 75, 79, 80, 83, 84, 93, 98, 104, $106,110]$. The model is based on the probabilistic interpretation of the laser pulse energy transfer by photons from the source to the detector and introduces the density of conditional probability $P\left[\mathbf{r}, t \mid\left(\mathbf{r}_{s}, t_{s}\right) \rightarrow\left(\mathbf{r}_{d}, t_{d}\right)\right]$ that the photon, migrating from the source spatio-temporal point $\left(\mathbf{r}_{s}, t_{s}\right)$ to the detector spatio-temporal point $\left(\mathbf{r}_{d}, t_{d}\right)$, at some intermediate time $t$ appears at the spatial point $\mathbf{r} \in V$. According to this model, the equation to be solved has the following integral form $[5,93,104,110]$ :

$$
\begin{aligned}
& g\left(\mathbf{r}_{s}, t_{s}, \mathbf{r}_{d}, t_{d}\right)= \\
& =\int_{V}\left[\begin{array}{l}
W_{\mu_{a}}\left(\mathbf{r}_{s}, t_{s}, \mathbf{r}_{d}, t_{d}, \mathbf{r}\right) \delta \mu_{a}(\mathbf{r})+ \\
+W_{D}\left(\mathbf{r}_{s}, t_{s}, \mathbf{r}_{d}, t_{d}, \mathbf{r}\right) \delta D(\mathbf{r})
\end{array}\right] d^{3} r,
\end{aligned}
$$

where

$$
W_{\mu_{a}}\left(\mathbf{r}_{s}, t_{s}, \mathbf{r}_{d}, t_{d}, \mathbf{r}\right)=c \int_{t_{s}}^{t_{d}} P\left[\mathbf{r}, t \mid\left(\mathbf{r}_{s}, t_{s}\right) \rightarrow\left(\mathbf{r}_{d}, t_{d}\right)\right] d t,
$$

$$
\begin{aligned}
& W_{D}\left(\mathbf{r}_{s}, t_{s}, \mathbf{r}_{d}, t_{d}, \mathbf{r}\right)=-\frac{1}{D} \int_{t_{s}}^{t_{d}} P\left[\mathbf{r}, t \mid\left(\mathbf{r}_{s}, t_{s}\right) \rightarrow\left(\mathbf{r}_{d}, t_{d}\right)\right] \times \\
& \times\left[c \mu_{a}+\frac{\partial}{\partial t} \ln G\left(\mathbf{r}-\mathbf{r}_{s}, t-t_{s}\right)\right] d t .
\end{aligned}
$$

Here $g\left(\mathbf{r}_{s}, t_{s}, \mathbf{r}_{d}, t_{d}\right)$ is the result of optical signal measurement, directly used for reconstruction; $c$ is the velocity of light in the object; $\delta \mu_{a}(\mathbf{r})$ and $\delta D(\mathbf{r})$ are the local spatial perturbations of the optical parameters $\mu_{a}$ and $D ; G\left(\mathbf{r}-\mathbf{r}^{\prime}, t-t^{\prime}\right)$ is the Green function of the nonstationary diffusion equation. The probability density $P\left[\mathbf{r}, t \mid\left(\mathbf{r}_{s}, t_{s}\right) \rightarrow\left(\mathbf{r}_{d}, t_{d}\right)\right]$ is also expressed in terms of the Green function. Thus, in the case of Robin boundary condition the following relation is valid [5, $57,93]$

$$
P\left[\mathbf{r}, t \mid\left(\mathbf{r}_{s}, t_{s}\right) \rightarrow\left(\mathbf{r}_{d}, t_{d}\right)\right]=\frac{G\left(\mathbf{r}-\mathbf{r}_{s}, t-t_{s}\right) G\left(\mathbf{r}_{d}-\mathbf{r}, t_{d}-t\right)}{G\left(\mathbf{r}_{d}-\mathbf{r}_{s}, t_{d}-t_{s}\right)} .
$$

For relatively small times of signal registration $\left(t_{d}-t_{s}<3000 \mathrm{ps}\right)$, according to Ref. [5, 98], one can use even simpler Dirichlet boundary condition, for which the probability density $P\left[\mathbf{r}, t \mid\left(\mathbf{r}_{s}, t_{s}\right) \rightarrow\left(\mathbf{r}_{d}, t_{d}\right)\right]$ can be presented as $[5,36,43,47,98,106,110]$

$$
\begin{aligned}
& P\left[\mathbf{r}, t \mid\left(\mathbf{r}_{s}, t_{s}\right) \rightarrow\left(\mathbf{r}_{d}, t_{d}\right)\right]= \\
& =\frac{G\left(\mathbf{r}-\mathbf{r}_{s}, t-t_{s}\right) \frac{\partial}{\partial q} G\left(\mathbf{r}_{d}-\mathbf{r}, t_{d}-t\right)}{\frac{\partial}{\partial q} G\left(\mathbf{r}_{d}-\mathbf{r}_{s}, t_{d}-t_{s}\right)},
\end{aligned}
$$

where $\partial / \partial q$ is the derivative in the direction normal to the medium boundary. Thus, for simple geometries, used in optical mammography and mammotomography, the weight functions $W_{\mu_{a}}\left(\mathbf{r}_{s}, t_{s}, \mathbf{r}_{d}, t_{d}, \mathbf{r}\right)$ and $W_{D}\left(\mathbf{r}_{s}, t_{s}, \mathbf{r}_{d}, t_{d}, \mathbf{r}\right)$ can be calculated analytically or semianalytically $[5,35,36,43,46,47,80,83,93,98$, 104, 106, 110]. Thus, e.g., in Ref. [106, 110] we proposed an approach in which the banana-shaped distributions of photon trajectories $W_{\mu_{a}}\left(\mathbf{r}_{s}, t_{s}, \mathbf{r}_{d}, t_{d}, \mathbf{r}\right)$ and $W_{D}\left(\mathbf{r}_{s}, t_{s}, \mathbf{r}_{d}, t_{d}, \mathbf{r}\right)$ are calculated analytically for the geometry of semi-infinite space. The corresponding distributions for transmission geometry of a plane layer are found using the original method of equivalent inverse source that makes use of the results, obtained for the semispace.

The measurement result, $g\left(\mathbf{r}_{s}, t_{s}, \mathbf{r}_{d}, t_{d}\right)$, referred by us to as the time-resolved optical projection $[5,93,98$, $104,106,110]$, is defined by the relation

$$
g\left(\mathbf{r}_{s}, t_{s}, \mathbf{r}_{d}, t_{d}\right)=-\ln \frac{\left.\Gamma\left(\mathbf{r}_{s}, t_{s}, \mathbf{r}_{d}, t\right)\right|_{t=t_{d}}}{\left.\Gamma_{0}\left(\mathbf{r}_{s}, t_{s}, \mathbf{r}_{d}, t\right)\right|_{t=t_{d}}}
$$

where $\Gamma\left(\mathbf{r}_{s}, t_{s}, \mathbf{r}_{d}, t\right)$ is the TPSF recorded by the detector $\mathbf{r}_{d}$ from the source $\mathbf{r}_{s}$ in the time-domain technique of data collection; the subscript " 0 " refers to the homogeneous scattering object unperturbed by the presence of optical inhomogeneities.

The crucial feature of Lyubimov model is that the time-resolved optical projection (23) is determined for a single time $t_{d}-t_{s}$ of signal registration. Hence, in principle, to reconstruct the image one has to know not the entire TPSF, but only one count of it. The timeresolved optical projections differ from the integral measurement data [5, 12, 224], used by the Western researchers, by the possibility to improve the spatial resolution via the choice of $t_{d}-t_{s}$ at the leading edge of the TPSF pulse. Thus, in Refs. [104, 106] it is shown that if for the reconstruction one uses the total bananashaped distributions of photon trajectories, defined by the relations (19) and (20), then it is possible to resolve features as small as $3 \mathrm{~mm}$ inside the object of size $8 \mathrm{~cm}$. This is confirmed by Fig. 9, presenting the result of reconstructing the model scattering object having the 
rectangular shape with the dimensions $10 \times 8 \mathrm{~cm}$ with two round absorbing inhomogeneities $3 \mathrm{~mm}$ in diameter. The optical parameters of the object have the following values: $\mu_{a}=0.05 \mathrm{~cm}^{-1}, D=0.034 \mathrm{~cm}$, and $n=1.4$. The inhomogeneities with the absorption coefficient $\mu_{a}=0.075 \mathrm{~cm}^{-1} a$ are located near the centre of the object and the separation between them is equal to the diameter. Figure 9 visualises the distribution $\delta \mu_{a}(\mathbf{r})$. Only the central part of the tomogram with the dimensions $5 \times 4 \mathrm{~cm}$ is shown. The relative drop between two peaks of the image intensity in Fig. 9 is nearly 65\%, which considerably exceeds the conditional level of $20 \%$ Rayleigh-Foucault visual resolution criterion. The obtained estimate of spatial resolution (better than 3 $\mathrm{mm})$ is even higher than the resolution of exact nonlinear multistep DOT algorithms. To solve the inverse problem we used the original modification [5, $80,83,84]$ of the known multiplicative algebraic reconstruction technique MART [243], allowing for the nonuniformity of distribution of the weight coefficients over the image domain.
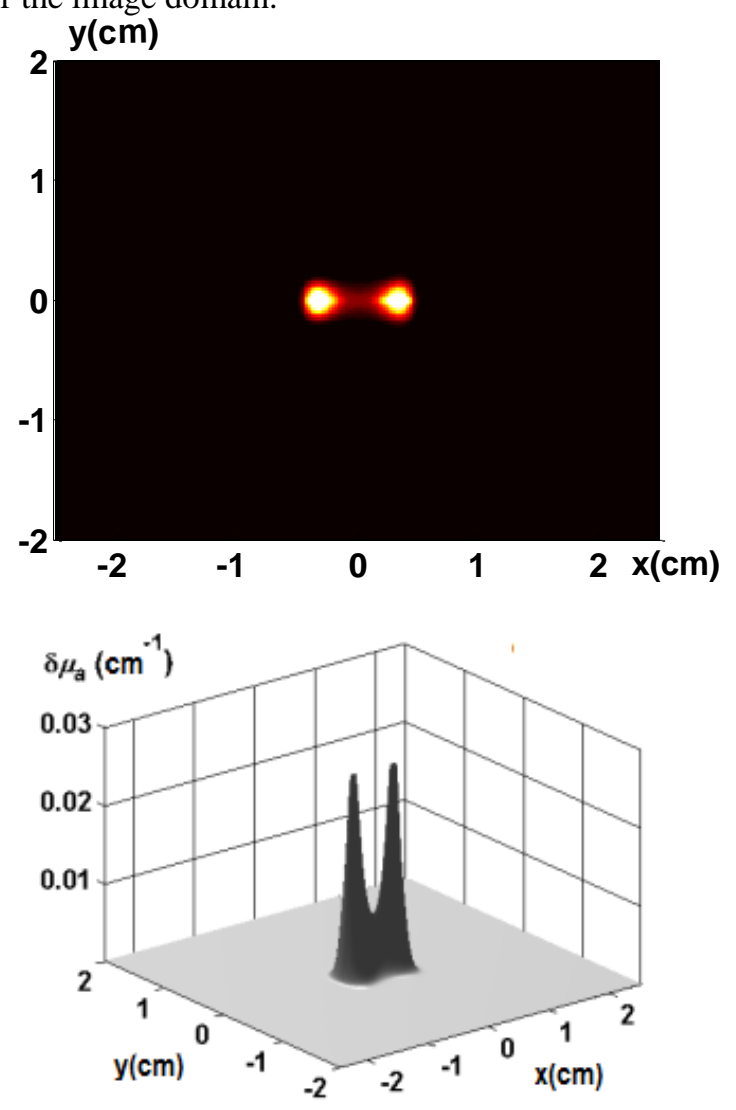

Fig. 9 Example of reconstruction of a numerical model with two absorbing inhomogeneities $3 \mathrm{~mm}$ in diameter.

As to the processing time, in Refs. [104, 106] it was not optimised. In the case of using the banana-shaped distributions the 2D reconstruction takes 30-50 minutes using the modified MART in the MATLAB environment at the personal computer with the processor 2.6-GHz Intel Core 2 Due having 4 GB RAM. According to our estimates, by optimising the code and using the software environment faster than MATLAB, the processing time can be reduced by several times. Of particular interest is also the application of objectoriented approach for the implementation of Lyubimov model using the GPUs. In this case, as follows from the experience of our colleagues [88, 91, 103, 107], the speed gain can be as large as 2 and more orders of magnitude.

Note, that the approach, analogous to the one described above, can be also implemented in DFMT, where for visualising the fluorophore lifetime they more and more frequently use the time-resolved measurement data [235, 267, 277-281]. In this case, the role of the time-resolved optical projection (23) can be played by the ratio of fluorescence and incident radiation fluxes, measured for individual counts of temporal fluorescence response and the TPSF, respectively [282].

To solve the inverse problem the IAP RAS team also uses linear methods, based on the inversion of the integral equation (15). In this case, the role of the measurement result $g\left(\mathbf{r}_{s}, \mathbf{r}_{d}\right)$ is played by the detected signal of fluorescence response, and the role of the object function $f(\mathbf{r})$ by the function of fluorophore distribution in the tissue. In Ref. [159] for the reconstruction of fluorophore distribution, they used an original method, generalising the standard algebraic technique ART [243] and based on the minimisation of the Holder norm. It was shown that for $p=1$ (the $L_{1}$ norm) the method allows the spatial resolution gain as compared to the ART that minimises the Euclidean norm. In Refs. [227, 228] I.I. Fiks proposed one more original inversion method based on the non-negativity condition of the fluorophore distribution function. The method named by the author as the method of Tikhonov functional with non-negative components, implies the transition from the SLAE to a system of bilinear algebraic equations solved by iterations. At each iteration, the Tikhonov regularisation is applied. The method was demonstrated to yield diffuse fluorescence tomograms of higher quality than the algebraic techniques ART and SMART [244], as well as the algorithm of non-negative least squares NNLS [239]. Thus, the studies performed at the experimental diffuse fluorescence tomograph designed in the IAP RAS confirmed the possibility of reliable resolution of two fluorescent inhomogeneities $3 \mathrm{~mm}$ in diameter, the separation between their centres being $6 \mathrm{~mm}$. However, the thickness of the scattering phantom, containing inhomogeneities, corresponded to the size of a small laboratory animal and amounted only to $2 \mathrm{~cm}$.

Ref. [143] presents the breast diffusion tomograms of voluntaries, obtained at the IAP RAS setup adapted for mammography studies. The tomograms visualise the spatial distributions of both optical and the functional parameters and confirm the possibility of reliable detection of cancer tumours (ductal carcinomas) with the size about one centimetre.

\subsection{Projection methods}

The reconstruction of diffusion mammograms in real time, during seconds or parts of a second, is now available only using the projection DOT methods. The 
rapid development of X-ray CT in the end of the 20-th century formed certain stereotypes in the opinion of medical society on how fast the tomogram reconstruction can be. Today using the electron beam tomograph of the 5-th generation it takes less than 50 milliseconds to get an X-ray 2D tomogram [283]. A 3D structure is reconstructed during 3-5 seconds. Apparently, such impressive figures are one of the reasons why the researchers working in the field of optical imaging still do not give up the attempts to apply the fast algorithms of projection tomography to DOT [38, 59, 66, 112, 114]. In mid-90s the company Philips (the Netherlands) even produced a commercial sample of diffusion mammotomograph using continuous-wave laser radiation, in which the breast image was reconstructed from blurred optical projections using the backprojection algorithm [112, 114]. However, the quality of resulting tomograms was poor, and Philips recently stopped the production of this tomograph. Projection methods provide only rough approximation of the real spatial distribution of optical inhomogeneities and yield not only to nonlinear, but also to perturbation semianalytic DOT methods in the precision of structure reproduction. First, this is due to the assumption of rectilinear light propagation underlying the projection methods. This assumption is invalid in principle, since in turbid media the photons have no regular trajectories. Second, the projection methods allow for the contribution to the optical signal only from the volume elements, crossed by the unique straight line, connecting the source and the detector. The contribution of other volume elements, crossed by complex trajectories of multiply scattered photons, is neglected. The experience by Philips showed that the rapid reconstruction with poor $(>6 \mathrm{~mm})$ resolution is inefficient in the diagnostics of breast cancer.

Thus, the projection approach to the reconstruction of diffusion tomograms should be cardinally modified to have prospects in clinical practice of cancer diagnostics. The modification should relate the basic assumptions that restrict the precision of reconstruction, such as the rectilinear light propagation and simplified calculation of the Jacobian matrix $\mathbf{J}$.

An example of such cardinal modification of the projection approach is the photon average trajectory (PAT) method developed by the researchers from SOI and RFNC-VNIITF [5, 15, 35, 36, 43, 46, 47, 57, 59, $75,79,80,83,84,98]$. Similar to all projection methods, this method reduces the inverse DOT problem to integration along a line, which ensures the fast tomogram reconstruction. However, in contrast to other projection methods, the PAT method operates not with straight lines, but with average statistical photon trajectories, curved near the boundaries of the scattering object. Thus, the PAT method allows for the character of light propagation near the boundaries thus essentially improving the accuracy of reconstructing the structures near the boundaries [80]. Moreover, the average trajectory consists of the points that give the maximal contribution to the recorded signal. Taking the contributions of these points (volume elements crossed by the photon average trajectory) into account allows the improvement of the spatial resolution of structures inside the object by 2 times as compared to the rough reconstruction along straight lines [104].

The PAT method is based on finding the most probable average statistical trajectory of a photon for each source-detector link. The trajectory is then used for reconstruction. Indeed, given the volume distribution of conditional probability density $P\left[\mathbf{r}, t \mid\left(\mathbf{r}_{s}, t_{s}\right) \rightarrow\left(\mathbf{r}_{d}, t_{d}\right)\right]$, one can find the centre of mass for this distribution at every moment of time $t \in\left[t_{s}, t_{d}\right]$ and, therefore, determine the trajectory along which this centre of mass moves. This trajectory is PAT. In the PAT method, the inverse DOT problem is reduced to the solution of the integral equation with integration along the curvy average trajectory, referred to as the fundamental equation of PAT method [57]. For absorbing inhomogeneities, this equation has the form

$$
g\left(\mathbf{r}_{s}, t_{s}, \mathbf{r}_{d}, t_{d}\right)=\int_{L} \frac{\left\langle\delta \mu_{a}(\mathbf{r})\right\rangle}{v(l)} d l
$$

where $\delta \mu_{a}(\mathbf{r})$ is the desired object function (absorbing inhomogeneity distribution function); $\langle\cdot\rangle$ denotes the operation of averaging over the spatial distribution of photons that contribute to the signal; $L$ is the photon average trajectory from the source point $\mathbf{r}_{s}$ to the detector point $\mathbf{r}_{d} ; l$ is the path length passed by the centre of mass of the distribution $P\left[\mathbf{r}, t \mid\left(\mathbf{r}_{s}, 0\right) \rightarrow\left(\mathbf{r}_{d}, t_{d}\right)\right]$ along the PAT to the time moment $t ; v(l)$ is the relative velocity of the centre of mass as a function of $l$. Eq. (24) is an analogue of Radon transform and can be resolved with respect to the function $<\delta \mu_{a}(\mathbf{r})>$ by means of fast algorithms of projection tomography. In particular, this is demonstrated in Ref. [57], where using the least-squares method with QR-factorisation [240], specially oriented to the inversion of SLAE with sparse matrices, the 2D reconstruction of the function $\left\langle\delta \mu_{a}(\mathbf{r})\right\rangle$ was performed during $0.5 \mathrm{~s}$, the record-breaking time for DOT. The spatial resolution of the PAT method in this case was $9 \mathrm{~mm}$. Later we improved the resolution to 6 $\mathrm{mm}[5,83,84,98]$ by using the original modification of the algebraic technique MART, already mentioned in Section 4.2. However, no further progress appeared possible. Apparently, the spatial resolution $\sim 6 \mathrm{~mm}$ is a limit, if the contribution to the recorded signal from the majority of the object points that do not lie on the PAT is not taken into account. In spite of relatively low resolution, to the opinion of the authors of the review, the PAT method can be successfully used to obtain "survey" images of breast in real time, similar to the use of topographical method in X-ray CT. 


\subsection{Methods approximately formulating the inverse problem of diffuse optical tomography}

Alongside with the methods described in Section 4.14.3 , at present a considerable variety of different methods and approaches to the solution of inverse DOT problem is developed, not aimed directly at the inversion of the operator equation (5) or integral equation (15). All such approaches and methods are inherently approximate from the point of view of setting the mathematical problem. We conditionally classify them as methods, approximately formulating the inverse DOT problem. As examples of such methods we can mention the inversion methods using neuron networks [284, 285], the nonlinear statistical methods developed by the MSU team [44, 53, 54, 58, 105] and, of course, the methods of localising optical inhomogeneities with regularisation $[10,74,92,99,101,102,109,113,115$, 121, 129-132, 208, 212].

The attempts of using artificial neuron networks for solving inverse problems on the base of detection of backscattered optical radiation are described in two papers by Russian researchers [284, 285]. In Ref. [284] it is shown that the medium absorption coefficient can be estimated with the error of $20 \%$, and the scattering coefficient and the anisotropy factor with the error of 5$10 \%$. Ref. [285] demonstrates the possibility to detect the optical inhomogeneity with the size $2-3 \mathrm{~mm}$ at the depth 3-5 $\mathrm{mm}$. To the opinion the authors of the review, the inversion methods based on neuron networks are of great interest for solving the problems of optical diagnostics. However, additional studies should be carried out to justify the efficiency of their application in clinical conditions as DOT methods.

The nonlinear statistical methods proposed by the MSU team are based on the hypothesis that the density of probability to detect the inhomogeneity $f_{i}(\mathbf{r})$ using the measured signal from the $i$-th source-detector link (below referred to as the $i$-th measurement) is proportional to the difference of photon fluxes $\Phi_{i}^{0}$ and $\Phi_{i}$, recorded for the homogeneous reference object and the object, perturbed by the presence of inhomogeneity. In this case, the following relation is valid:

$$
f_{i}(\mathbf{r})=\frac{\Phi_{i}^{0}-\Phi_{i}}{\Phi_{i}^{0}} p_{i}^{0}(\mathbf{r})
$$

where $p_{i}^{0}(\mathbf{r})$ is the probability density of photons of the i-th measurement crossing the arbitrary point $\mathbf{r}$ of the reference object. The function $p_{i}^{0}(\mathbf{r})$ is calculated approximately using the Monte Carlo method and different methods of approximation [44, 53, 54]. The probability density to detect the inhomogeneity from the results of all $N$ measurements is calculated using the formula

$$
f_{\Sigma}(\mathbf{r})=\sum_{i}^{N} f_{i}(\mathbf{r})
$$

In fact, the function $f_{\Sigma}(\mathbf{r})$ plays the role of the object function, i.e., the function that describes the inner structure of the object. Note, that there is some analogy with the backprojection algorithm in absorption tomography [283], when the result of reconstruction is obtained by summation of all projections, interpolated on the image mesh. The algorithm (25), (26) implies strong dependence of the reconstruction result upon the a priori knowledge of the reference object. To remove this drawback, the authors of [54, 58, 105] proposed two modifications of the algorithm. One of them [54, 58] implies the introduction of iterative procedure, according to which at each next iteration the functions $\Phi_{i}^{0}$ and $p_{i}^{0}(\mathbf{r})$ are corrected using the results of the previous one. In Ref [105] the authors proposed using a differential measurement scheme and recording the optical signal at two different wavelengths $\lambda_{1}$ and $\lambda_{2}$. In this case, in Eq. (25) instead of the difference $\Phi_{i}^{0}-\Phi_{i}$ one can use the difference of the measured fluxes $\Phi_{i}\left(\lambda_{1}\right)-\Phi_{i}\left(\lambda_{2}\right)$, and thus minimise the dependence of the result of reconstruction upon the reference object.

In spite of the absence of rigorous mathematical substantiation, the nonlinear statistical methods are undoubtedly attractive, and, above all, allow the reconstruction of structures with high spatial resolution $\sim 3 \mathrm{~mm}$ in the object with the diameter $14 \mathrm{~cm}$. Actually, such resolution was obtained for the structures with $100 \%$ contrast, i.e., the absolutely absorbing inhomogeneities. Unfortunately, the nonlinear statistical methods are unable to separate reliably the spatial distributions of the optical parameters $\mu_{a}$ and $\mu_{s}^{\prime}$, which makes it impossible to use them in functional diagnostics of tissues.

The inhomogeneity localisation methods are the fitting methods based on the comparison of experimental data with the calculated ones, obtained from the analytical solution of the diffusion equation for homogeneous medium with a spherical inhomogeneity. For the aims of optical mammography, they usually apply the planar layer transmission geometry and the time-domain technique of tissue probing and signal detection. The classical approach described, e.g., in Ref. [115], can be conditionally divided into three stages. At the first stage the breast is assumed to have homogeneous structure, and the mean values of the optical parameters $\mu_{a}$ and $\mu_{s}^{\prime}$ are estimated using the results of comparing the measured and calculated photon fluxes, transmitted through the layer. At the second stage the tumour with the optical parameters, the localisation, and the size to be determined, is modelled by a spherical inhomogeneity. To derive the analytical relations, the model of diffraction of spherical waves of photon density, rounding a spherical obstacle is used [286]. The solution is constructed in the frequency domain using the image method [287], and then to find 
the photon fluxes perturbed by the presence of the inhomogeneity, the transition to the time domain is performed. To compare the measured and calculated fluxes, the least-squares method is commonly used. At the third stage, the measured TPSFs are used to form optical mammograms.

The method of inhomogeneity localisation, in fact, yields no information on the real shape of the tumour. It allows one to determine only the place of its localisation and the "effective" size. However, such methods with certain success are used in experimental clinical studies to determine optical parameters of breast tissues in vivo [115, 121, 129-132, 208, 212].

Most recently, the methods of inhomogeneity localisation acquired further development at TSTU [92, 99, 102, 109]. In these papers the authors propose that the localisation problem should be solved using latearriving photons that form the trailing edge of the TPSF. In Refs. [92, 99] the numerical and nature experiments were used to show that the time dependence of TPSF logarithm has an asymptote, the position of which is determined exclusively by the late-arriving photons. From the geometric characteristics of this straight line one can draw conclusions about the optical parameters $\mu_{a}$ and $\mu_{s}^{\prime}$ of the scattering object, for which the measurements are carried out. If, e.g., for a cylindrical object with an inhomogeneity the measurements of TPSF are performed at different angular positions of the detector, then, analysing the position of appropriate asymptotes, one can estimate the localisation of the inhomogeneity, as well as its size and optical parameters. In Ref. [102] in order to localise the inhomogeneity it was proposed the use of the 3D visualisation of the set of TPSF, where the role of the 3rd dimension is played by the angle $\alpha$ between the source fibre and the detector fibre. From the curvature of the obtained 3D surface one can estimate the size of the inhomogeneity and the approximate depth of its localisation. Finally, in Ref. [109] the authors used a two-step procedure to make the localisation more precise. At the first step, using the so-called inhomogeneity index, which represents the dependence of the mean flux density of late-arriving photons upon the angle $\alpha$, the initial approximation of the image of the cylindrical object section with inhomogeneity is formed. At the second step, the optical and geometric characteristics of the inhomogeneity are specified by iterative minimisation of a certain objective function. Note, that the time of specified image formation using the workstation HPZ640 with two six-core processors E5-2620v3c and 32 GB RAM did not exceed 3 seconds.

\section{Conclusion}

In the present review, we discuss the state-of-the-art of diffuse optical mammotomography, the promising method of breast cancer diagnostics. The review of diffusion tomography instrumentation is presented, as well as of the modern methods of reconstructing spatial distributions of optical and functional parameters. It is shown that the problem of developing the instrumentation for a diffuse optical mammotomograph at present is actually solved. To date the only obstacle for entering the market of medical imaging facilities is the absence of fast reconstruction algorithms, capable of reconstructing the diffusion tomograms with required precision in real time. However, the present-day development level of methodological base for diffusion tomography alongside with the rapid progress of computer technologies allows us to suggest that this obstacle will be removed in a few years. Thus, the multistep nonlinear methods and some perturbation methods of reconstruction allow the achievement of resolution, very close to that required by oncologists (features smaller than $4 \mathrm{~mm}$ ). The next step in the progress of processor techniques will solve the problem of reconstruction time. Thus, one can expect the appearance of commercial diffuse optical tomographs specialised for the breast cancer diagnostics in the nearest future.

The contribution of Russian scientists to the development of methodological base of the diffusion optical tomography is worth adequate appraisal. The methods developed by them, namely, the inversion methods using the perturbation Lyubimov model, the algebraic method for minimising the Holder norm, the method of Tikhonov functional with non-negative components, the nonlinear statistical methods, and the method of inhomogeneity localisation, based on detecting late-arriving photons, can occupy a deserved place among other methods of solving the inverse problem of diffusion tomography. In view of the prospects of the optical mammotomography, we particularly mention the contribution of SOI and RFNCVNIITF teams. Thus, one of the implementations of Lyubimov perturbation model allowed the recordbreaking spatial resolution (features smaller than $3 \mathrm{~mm}$ in the object of size about $8 \mathrm{~cm}$ ). The other implementation allowed the record-breaking reconstruction time $(0.5 \mathrm{~s})$. One can expect that if in the nearest future it becomes possible to perform clinical testing of the developed methods, then the Russian producers will be able to offer a competitive product for the market of commercial diffuse optical tomographs. We emphasise that potentially the value of $3 \mathrm{~mm}$ corresponds to the size of a cancer tumour when it may be not yet invasive, so that its detection allows the hope for successful healing of the patient. That is why one can expect that the DOT methods based on the perturbation Lyubimov model will be efficient in the diagnostics of oncologic diseases of breast just at the early stage of their development.

\section{Acknowledgements}

ANB and EAG were supported by Russian Presidential grant NSh-7898.2016.2. 